\title{
All minor-minimal apex obstructions with connectivity two
}

\author{
Adam S. Jobson André E. Kézdy \\ Department of Mathematics \\ University of Louisville \\ Louisville, Kentucky, U.S.A. \\ kezdy@louisville.edu
}

Submitted: Dec 14, 2018; Accepted: Jan 5, 2021; Published: Jan 29, 2021

(C) The authors. Released under the CC BY-NC-SA license (International 4.0).

\begin{abstract}
A graph is an apex graph if it contains a vertex whose deletion leaves a planar graph. The family of apex graphs is minor-closed and so it is characterized by a finite list of minor-minimal non-members. The long-standing problem of determining this finite list of apex obstructions remains open. This paper determines the 133 minorminimal, non-apex graphs that have connectivity two.
\end{abstract}

Mathematics Subject Classifications: 05C10, 05C83

\section{Introduction}

A graph is an apex graph if it contains a vertex, called an apex, whose deletion leaves a planar graph. The family of apex graphs is minor-closed and so Robertson and Seymour's graph minor theorem [16] implies that this family has a finite list of minor-minimal non-members (also known as obstructions). Despite considerable efforts for decades, the problem of determining the list of planar apex obstructions remains open. In this paper we determine the 133 minor-minimal non-apex graphs that have connectivity two.

Apex graphs play a key role in what is commonly now referred to as the "weak structure theorem" of the Robertson and Seymour's graph minors project (recently this "weak structure theorem" has been optimized by Giannopoulou and Thilikos [10]). Apex graphs have also featured prominently in the resolution of Hadwiger's conjecture for $K_{6}$-free graphs [17] and the characterization of linklessly embeddable graphs. The latter problem highlights the Petersen family of graphs [19], a significant collection of apex obstructions. Even with advances in algorithmic refinements of the graph minors project that are focused on determining obstruction sets for apex families $[2,14]$, the very general and 
theoretical approaches are frustratingly impractical. Disconcertingly the problem of determining all apex-planar obstructions remains open, despite classical linear-time planarity testing algorithms and dramatic increases in computing power.

Researchers have made progress characterizing families of graphs that are closely related to the apex graphs. An old result of Wagner [4] characterizes the "almost planar" graphs; a graph that is non-planar but the deletion of any vertex makes it planar. The terms "almost planar" and "nearly planar" appear in many articles with a dizzying variety of meaning. Gubser [11] characterizes another family of graphs with another notion of "almost planar" - a non-planar graph such that for any edge, either the contraction or the deletion of that edge makes the graph planar (see also [6]). Ding and Dzobiak $[5,7]$ determined the 57 graphs that are the obstructions for the minor-closed family of apex-outerplanar graphs.

It has been a long standing open problem to determine the apex obstructions. At a conference in 1993, the second author discussed the apex obstruction problem with Robin Thomas. Together with Daniel Sanders we decided to share lists of known apex obstructions; the combined list contained 123 graphs at that time. Twenty years later we reported 396 known apex obstructions [13]. Our list has since grown to 401 nonisomorphic apex obstructions. Other groups have relayed the problem or worked to find obstructions $[1,3,8,9,12,15]$. Indeed we credit David Eppstein (see bottom entry of [9]) for finding one particularly beautiful 16-vertex apex obstruction. He describes it this way: "start with a cube, find a four-vertex independent set, and make three copies of each of its vertices. The resulting 16 -vertex graph has four $K_{3,3}$ subgraphs, one for each tripled vertex." We refer to this obstruction as the Eppstein graph.

In this paper we determine the 133 minor-minimal non-apex graphs that have connectivity two. It is straightforward to prove there are three disconnected apex obstructions and no apex obstructions with connectivity one (see the beginning of section 3 for details). Determining all apex obstructions that have connectivity greater than two is the focus of future research. Lipton et al. [12] have shown that apex obstructions have connectivity at most five. Our proof here presents the connectivity-2 apex obstructions in five groups (see Figure 1); this is essentially the same argument we presented at the AMS Meeting in 2013 [13]. Section 2 introduces basic notation and definitions. Section 3 presents elementary observations about apex obstructions. Sections 4-7 present a general outline and resolve four of the five groups of obstructions.

Section 8 considers the last group of obstructions, the 72 connectivity- 2 apex obstructions with a unique 2-cut having a planar heavy component (defined in the paragraph before Lemma 9). These obstructions are considerably more difficult to characterize because they are very close to 3-connected. One aspect of connectivity-2 apex obstructions is that they may contain vertices that are not branch vertices of any Kuratowski subdivision. There are seven obstructions exhibiting this phenomenon, three appear at the bottom of Figure 5 and four appear at the bottom of Figure 8. This is not a phenomenon encountered in general 3-connected graphs that contain a subdivision of $K_{3,3}$ (see statement (6.2) of [19]). The final 72 connectivity-2 apex obstructions behave similarly to the 3 -connected graphs that contain a subdivision of $K_{3,3}$ : all vertices are branch vertices 
of a Kuratowski subdivision. Proposition 21 is the main tool used to prove this claim; it highlights the significance of "close" Kuratowski subdivisions (which we do not define in this paper), a notion remotely reminiscent to 'communicating Kuratowski subgraphs' (introduced in [18]). The most important consequence of Proposition 21 is that the apex obstructions having a unique 2-cut with a planar heavy component contain three Kuratowski subdivisions whose branch vertices cover the entire vertex set. This property is key in our proof that the obstruction list is complete. It appears likely that the study of apex obstructions with higher connectivity will require similar collections of Kuratowski subdivisions whose branch vertices cover the entire vertex set, though the Eppstein graph shows that as many as four such Kuratowski subdivisions are needed. Unfortunately connectivity appears to be a poor proxy for a still missing notion related to close complexes of Kuratowski subdivisions. After reducing to small graphs the computation of the 72 connectivity-2 apex obstructions having a unique 2-cut with a planar heavy component, the final subsection discusses the computer work applied to show the list of connectivity2 apex obstructions is complete (see Appendix A for graph6 presentations of all 133 connectivity-2 apex obstructions).

Because it may be of independent interest, Section 9 presents another interpretation of the characterization of connectivity-2 apex obstructions in terms of double apex graphs.

\section{Preliminaries}

All graphs in this paper are finite, simple, and undirected. The set of vertices of the graph $G$ is denoted $V(G)$, which is often abbreviated to $V$. Similarly $E(G)$, or simply $E$, denotes the set of edges of $G$. Edges are unordered pairs of vertices, but following standard notation, the edge $e=\{a, b\}$ is abbreviated $a b$. The vertices $a$ and $b$ are the endpoints of the edge $e=a b$. The edge $e=a b$ is incident to $a$ and $b$. Incidence is often written as membership, as when $a \in e$ signals that edge $e$ is incident with vertex $a$. The neighbors of a vertex $u$ in the graph $G$ is the set $N(u)=\{v \in V: u v \in E\}$, which is sometimes denoted $N_{G}(u)$ to emphasize the graph. For any $v \in V(G)$, the set of edges incident to $v$ is denoted $E_{v}=\{e \in E(G): v \in e\}$. Following standard notation, $d_{G}(v)$, $\delta(G), \kappa(G)$ denote the degree of the vertex $v$ in $G$, the minimum degree of a vertex in $G$, and the vertex connectivity of $G$, respectively. A $u v$-path in $G$ is a path whose endpoints are $u$ and $v$. If $S$ is collection of vertices, then $G[S]$ denotes the subgraph of $G$ induced by $S$.

Often, when the meaning is clear, mathematical elements are recast and operations are overloaded for notational convenience. So, for example, the graph induced by $V(G)-S$ is denoted simply $G-S$, which replaces the more syntactically accurate but cumbersome $G[V(G)-S]$, thereby overloading the subtraction operator. Furthermore, in the case that $v$ is a vertex, $G-\{v\}$ is abbreviated to $G-v$, which implicitly recasts a vertex as a set. Recasting graphs as their vertex sets (and vice versa) appears frequently. For example, if $A$ and $B$ are subgraphs of $G$, then $A \subseteq V(G)-B$ tacitly recasts $A$ and $B$ as their vertex sets and is short for $V(A) \subseteq V(G)-V(B)$ since it does not make sense to subtract a graph from a vertex set. But note carefully that $A \subseteq V(G)-B$ is very different in 
meaning from $A \subseteq G-B$; in the latter expression no recasting takes place because all variables represent graphs and subtraction makes sense. Other operators are overloaded naturally as needed. For example, following standard notation, the addition operator can be applied to (possibly recast) sets of edges or (possibly recast) sets of vertices. For example, $G+e$ represents the graph resulting from the addition of the edge $e$ to the graph $G$. Similar conveniences appear throughout.

The number of components in $G-S$ is $c(G-S)$. A vertex set $S$ is a cutset for a graph $G$ if $c(G)<c(G-S)$. A cutset with $k$ vertices is called a $k$-cut. A cutset $S$ for $G$ is said to separate vertices $u$ and $v$ if $u$ and $v$ are in the same component of $G$ but different components of $G-S$. A path of $G$ crosses a cutset $S$ if it contains vertices from two different components of $G-S$. If $S=\{v\}$ is a cutset for $G$, then $v$ is a cut vertex, also known as a 1-cut. If $H$ is a subgraph of a component of $G-S$, then the $H$-side (of $G-S)$ is the component of $G-S$ that contains $H$.

A subdivision of $K_{5}$ or $K_{3,3}$ is called a Kuratowski subgraph. Kuratowski proved that a graph is non-planar if and only if it contains a Kuratowski subgraph. A vertex of a Kuratowski subgraph is a branch vertex if its degree in the Kuratowski subgraph is at least 3. A vertex of a Kuratowski subgraph that is not a branch vertex is also called a subdividing vertex or a non-branch vertex.

The contraction of an edge $e$ in the graph $G$ produces a graph denoted $G / e$. A graph $G$ contains $H$ as a minor, denoted $H \leqslant_{m} G$, if a subgraph isomorphic to $H$ can be obtained from a subgraph of $G$ by a sequence of edge contractions. Observe that the minor order is transitive. A family $\mathcal{F}$ is minor-closed if $G \in \mathcal{F}$ and $H \leqslant_{m} G$ implies that $H \in \mathcal{F}$. If $\mathcal{F}$ is a minor-closed family, then the minor-minimal graphs that are not in $\mathcal{F}$ are called obstructions; so an obstruction is a graph $G \notin \mathcal{F}$ such that $H \in \mathcal{F}$, for all $H \leqslant_{m} G$ and $H \neq G$.

The disjoint union of graphs $G$ and $H$ is denoted $G+H$. Also $2 G$ abbreviates $G+G$.

\section{Simple observations}

A graph is an apex graph if it contains a vertex, called an apex, whose deletion produces a planar graph. Every planar graph is an apex graph. The family of apex graphs is minorclosed and so it has finite list of minor-minimal non-members, also known as forbidden minors or obstructions. Let $\mathcal{F}$ denote the finite set of obstructions for apex graphs.

We begin with a few simple observations about graphs in $\mathcal{F}$.

Lemma 1. If $G \in \mathcal{F}$, then $\delta(G) \geqslant 3$.

Proof. Suppose, to the contrary, that there exists $G \in \mathcal{F}$ and $v \in V(G)$ with $d_{G}(v) \leqslant 2$. If $d_{G}(v) \leqslant 1$, then $G-v$ is apex with an apex, $w$. Note that $G-v-w$ is planar which implies that $G-w$ is also planar, contradicting $G \in \mathcal{F}$. If $d_{G}(v)=2$, then consider an edge $e$ incident to $v$. The contraction of $e$ produces an apex graph $G / e$ with an apex vertex, $w$. Now $G / e-w$ is planar which implies that $G-w$ is planar, contradicting $G \in \mathcal{F}$. 
Lemma 2. The disconnected graphs in $\mathcal{F}$ are $2 K_{5}, 2 K_{3,3}$, and $K_{5}+K_{3,3}$.

Proof. The reader can easily verify that $2 K_{5}, 2 K_{3,3}$, and $K_{5}+K_{3,3}$ are disconnected graphs in $\mathcal{F}$. It suffices to show there are no others. Consider a disconnected graph $G \in \mathcal{F}$. Each component of $G$ must be an apex graph since removing any one edge from $G$ produces an apex graph. There must be at least two non-planar components since otherwise the whole graph is an apex graph. It follows that there are exactly two components and each is non-planar apex. Consider an arbitrary component of $G$, call it $C$. Let $H$ be a Kuratowski subgraph in $C$. If there is an edge $e \in E(C)-E(H)$, then $G-e$ is not apex, contradicting that $G \in \mathcal{F}$; hence, $E(H)=E(C)$. Lemma 1 guarantees $\delta(G) \geqslant 3$, from which it follows that all the vertices of $C$ are branch vertices of $H$; consequently, $C \cong K_{5}$ or $C \cong K_{3,3}$.

Lemma 3. Every connected graph in $\mathcal{F}$ has connectivity at least two.

Proof. Suppose, to the contrary, that $G \in \mathcal{F}$ and $\kappa(G)=1$. Let $v$ be a cut vertex of $G$. Now $G-v$ is non-planar; therefore, there exists a Kuratowski subgraph, $H$, in one of the components, say component $C$, of $G-v$. Define $T=\{u v \in E(G): u \in V(C)\}$. Because $G-T$ is apex with apex vertex in $V(H) \subseteq V(C)$, it follows that $G-C$ is planar. Let $e$ be an edge incident to $v$ that is also incident to a vertex in a component of $G-v$ other than $C$. Because $G-e$ is apex, there is an apex of $G-e$, say $w$, that is in $V(H) \subseteq V(C)$. Because $G[V(C) \cup\{v\}]$ is apex (with apex $w$ ) and $G-C$ is planar, it follows that $G-w$ is planar: embed $G[V(C) \cup\{v\}]-w$ in the plane with $v$ on the exterior face, separately embed $G-C$ in the plane with $v$ on the exterior face, and identify $v$ 's in these embeddings, producing a planar embedding of $G-w$. This contradicts that $G$ is not an apex graph.

Because of Lemmas 2 and 3, we now consider only obstructions in $\mathcal{F}$ that have connectivity at least two. Indeed, in this paper we determine all graphs $G \in \mathcal{F}$ such that $\kappa(G)=2$; there are precisely 133 of them.

For $G \in \mathcal{F}$ and $v \in V(G)$, let $H_{v}$ denote a Kuratowski subgraph in $G-v$. Note that $H_{v}$ witnesses that $G$ is not apex; it is a Kuratowski witness for $v$.

If $S \subseteq V$ is a cutset for $G$ and $C$ is a component of $G-S$, then the augmentation of $C$ is

$$
C^{+}=G[V(C) \cup S]+\{u v: u, v \in S\} .
$$

This is also referred to as the augmented component of $G-S$ obtained from $C$.

The next lemma gathers several elementary properties of Kuratowski witnesses.

Lemma 4. Suppose $G \in \mathcal{F}$ and $\kappa(G) \geqslant 2$. For all $u, v, w \in V(G)$, and all Kuratowski witnesses $H_{u}, H_{v}$ and $H_{w}$ for $u, v$ and $w$, respectively,

i) $V\left(H_{u} \cap H_{v}\right) \neq \varnothing$,

ii) if $V\left(H_{u} \cap H_{v} \cap H_{w}\right)=\varnothing$, then $E(G)=E\left(H_{u} \cup H_{v} \cup H_{w}\right)$, 
iii) if $V\left(H_{u} \cap H_{v} \cap H_{w}\right)=\varnothing$ and $x \in V\left(H_{u}\right)-V\left(H_{v} \cup H_{w}\right)$, then $x$ is a branch vertex of $H_{u}$,

iv) if $\kappa(G)=2, S$ is a 2-cut of $G$, and $s \in S$, then for any Kuratowski witness $H_{s}$, there exists an augmented component $C^{+}$of $G-S$ such that $V\left(H_{s}\right) \subseteq V\left(C^{+}\right)$.

Proof. i) If $V\left(H_{u} \cap H_{v}\right)=\varnothing$, then $G=H_{u}+H_{v}$ is a disconnected obstruction in $\mathcal{F}$. $\left.i i\right)$ Assume $V\left(H_{u} \cap H_{v} \cap H_{w}\right)=\varnothing$. If there were an edge $e \in E(G)-E\left(H_{u} \cup H_{v} \cup H_{w}\right)$, then $G-e$ would have no apex. iii) Suppose $V\left(H_{u} \cap H_{v} \cap H_{w}\right)=\varnothing$ and $x \in V\left(H_{u}\right)-V\left(H_{v} \cup H_{w}\right)$. Assume, to the contrary, that $x$ is not a branch vertex of $H_{u}$; so $d_{H}(x)=2$. Lemma 1 implies $d_{G}(x) \geqslant 3$. Consequently there is at least one edge $e$ incident to $x$ that does not belong to $E\left(H_{u} \cup H_{v} \cup H_{w}\right)$, contradicting that $G-e$ has an apex. iv) Assume $G \in \mathcal{F}$, $\kappa(G)=2, S$ is a 2-cut of $G$, and $s \in S$. Consider a Kuratowski witness $H_{s}$. Observe that some augmented component of $G-S$ must contain all the vertices in $V\left(H_{s}\right)$ because no path of the 2-connected subgraph $H_{s}$ can cross the 1-cut $S-s$.

Lemma 5. Suppose that $G \in \mathcal{F}, \kappa(G)=2$, and $S=\{a, b\}$ is a 2-cut of $G$. Every augmented component $C^{+}$of $G-S$ contains an edge $e \in E\left(C^{+}\right)-a b$ such that there exists an ab-path in $C^{+}-e-a b$.

Proof. Consider an arbitrary component of $G-S$, call it $C$, and its augmentation $C^{+}$. Let $P$ be an $a b$-path in $C^{+}-a b$ ( $P$ exists because $S$ is a minimum cutset of $G$ ). The path $P$ contains a vertex $v \notin S$. Let $e$ be an edge incident to $v$ that is not in $P$; the existence of such an edge follows from Lemma 1. Now $e$ is the desired edge in $E\left(C^{+}\right)-a b$.

Next we present a technical lemma that will be applied in several upcoming arguments.

Lemma 6. Suppose that $G \in \mathcal{F}, \kappa(G)=2, S=\{a, b\}$ is a 2-cut of $G$, and $C$ is a component of $G-S$. If $e \in E\left(C^{+}\right)-a b$ and there exists an ab-path in $C^{+}-e-a b$, then for any apex $w$ of $G-e$ and any Kuratowski subgraph $H_{w}$ in $G$ avoiding $w$, the branch vertices of $H_{w}$ are all in $V\left(C^{+}\right)$.

Proof. Because $G-e-w$ is planar, it follows that $e \in E\left(H_{w}\right)$. All of the branch vertices of $H_{w}$ must be in the same augmented component of $G-S$ because $|S|=2$ but $H_{w}$ is a subdivision of $K_{5}$ or $K_{3,3}$ which are 3-connected. If the branch vertices of $H_{w}$ are not in $V\left(C^{+}\right)$, then $e$ appears in $H_{w}$ only as an edge along a path connecting $a$ and $b$. Consequently, a Kuratowski subgraph in $G-w-e$ would exist by replacing this $a b$-path in $C^{+}$by another $a b$-path from $C^{+}-e-a b$ (which exists by assumption), contradicting that $w$ is an apex vertex for $G-e$. So the branch vertices of $H_{w}$ must be in $V\left(C^{+}\right)$.

Recall that, if $S \subseteq V$ is a cutset for $G$, then $c(G-S)$ is equal to the number of components in $G-S$.

Lemma 7. If $G \in \mathcal{F}, \kappa(G)=2$, and $S$ is a 2-cut of $G$, then $c(G-S)=2$. 
Proof. Assume, to the contrary, that $c(G-S)>2$. Let $S=\{a, b\}$ and let $C_{1}, C_{2}, C_{3}$ be three components of $G-S$. Applying Lemma 4 part $i v)$, we may assume that $V\left(H_{a}\right) \subseteq C_{1}^{+}$. It follows from Lemma 4 parts i) and $i v)$ that $V\left(H_{b}\right) \subseteq C_{1}^{+}$. Lemma 5 guarantees there exists an edge $e$ in $E\left(C_{3}^{+}\right)-a b$ such that an $a b$-path remains in $C_{3}^{+}-a b-e$. Because $G \in \mathcal{F}$, the graph $G-e$ is an apex graph; let $w$ be an apex for $G-e$. Consider $H_{w}$, a Kuratowski subgraph avoiding $w$. By Lemma 6 the branch vertices of $H_{w}$ must be in $V\left(C_{3}^{+}\right)$.

Now Lemma 5 guarantees there exists an edge $f \in E\left(C_{2}^{+}\right)-a b$ such that an $a b$-path remains in $C_{2}^{+}-a b-f$. Notice that if $f$ is an edge in $H_{w}$ then, because the branch vertices of $H_{w}$ must be in $V\left(C_{3}\right)$, the edge $f$ appears in $H_{w}$ only as an edge along an $a b$-path in $C_{2}^{+}-a b$. Consequently there is a Kuratowski subgraph $H_{w}^{*}$ in $G\left[V\left(C_{2} \cup C_{3}\right) \cup\{a, b\}\right]$ that avoids the edge $f$; it is obtained from $H_{w}$ by replacing, if necessary, any $a b$-path in $H_{w} \cap\left(C_{2}^{+}-a b\right)$ by an $a b$-path in $C_{2}^{+}-a b-f$. This implies that $H_{a}, H_{b}$ and $H_{w}^{*}$ share no common vertex, contradicting $G-f$ is apex.

Lemma 8. If $G \in \mathcal{F}, \kappa(G)=2$, and $S$ is any 2-cut of $G$, then the augmentation of any component of $G-S$ is non-planar.

Proof. Let $S=\{a, b\}$ be an arbitrary 2-cut of $G$. Lemma 7 guarantees that $G-S$ has exactly two components, call them $C_{1}$ and $C_{2}$. Applying Lemma 4 part $i v$ ), we may assume that $H_{a} \subseteq C_{1}^{+}$. It follows from Lemma 4 parts $i$ ) and $\left.i v\right)$ that $H_{b} \subseteq C_{1}^{+}$. It suffices to prove that $C_{2}^{+}$is non-planar. Lemma 5 guarantees there exists an edge $e \in E\left(C_{2}^{+}\right)-a b$ such that an $a b$-path remains in $C_{2}^{+}-a b-e$. Because $G \in \mathcal{F}$, the graph $G-e$ is an apex graph; let $w$ be an apex for $G-e$ and let $H_{w}$ be a Kuratowski subgraph of $G$ avoiding $w$. By Lemma 6 the branch vertices of $H_{w}$ must be in $V\left(C_{2}^{+}\right)$. This implies that $H_{w} \subseteq C_{2}^{+}$, so $C_{2}^{+}$is non-planar.

Consider $G \in \mathcal{F}$ with $\kappa(G)=2$ and $S=\{a, b\}$ any 2-cut of $G$. By Lemma 7, there are only two components of $G-S$, call them $C_{1}$ and $C_{2}$. We may assume that $H_{a} \subseteq C_{1}^{+}$ and $H_{b} \subseteq C_{1}^{+}$. We call $C_{1}$ the heavy component of $G-S$ because $H_{a}, H_{b} \subseteq C_{1}^{+} ; C_{2}$ is the light component. A 2-cut of $G$ is basic if the vertex set of its heavy component is minimal (with respect to set inclusion); that is, no 2-cut produces a heavy component whose vertex set is properly contained in this one.

Lemma 9. If $G \in \mathcal{F}, \kappa(G)=2$, and $S$ is any basic 2-cut of $G$, then the augmentation of the light component of $G-S$ is isomorphic to $K_{5}, K_{3,3}$, or $K_{3,3}+e$.

Proof. Let $S=\{a, b\}$ be an arbitrary basic 2-cut of $G$. Let $C_{1}$ and $C_{2}$ be the two components of $G-S$, with $C_{1}$ the heavy component and $C_{2}$ the light one. Lemma 5 guarantees there exists an edge $e \in E\left(C_{2}^{+}\right)-a b$ such that an $a b$-path remains in $C_{2}^{+}-a b-e$. Because $G \in \mathcal{F}$, the graph $G-e$ is an apex graph. Let $w$ be an apex for $G-e$ and let $H_{w}$ be a Kuratowski subgraph of $G$ avoiding $w$. Observe $w \in V\left(H_{a} \cap H_{b}\right) \subseteq V\left(C_{1}\right)$. By Lemma 6 the branch vertices of $H_{w}$ must be in $V\left(C_{2}^{+}\right)$. Furthermore, there must be some part of $H_{w}$ that is not in $C_{2}$ since $V\left(H_{w} \cap H_{a}\right) \neq \varnothing$ and $V\left(H_{w} \cap H_{b}\right) \neq \varnothing$, so in particular, $\{a, b\} \subseteq V\left(H_{w}\right)$. 
Claim: $w$ is an apex for $G-f$ and $G / f$, for all $f \in E\left(C_{2}^{+}\right)-\{a b\}$.

Suppose, to the contrary, there is an edge $f \in E\left(C_{2}^{+}\right)-\{a b\}$ such that $w$ is not an apex for $G-f$ or $G / f$. There must be an apex vertex for $G-f$ and $G / f$. Let $z$ be an apex for $G-f$ if $w$ is not an apex for it; otherwise let $z$ be an apex for $G / f$. Clearly $z \in V\left(H_{a} \cap H_{b} \cap H_{w}\right) \subseteq V\left(C_{1}\right)$ and $z \notin\{a, b, w\}$. Because the branch vertices of $H_{w}$ are in $C_{2}^{+}$and $z \notin\{a, b\}$, it follows that $H_{w} \cap G\left[V\left(C_{1}\right) \cup\{a, b\}\right]$ is an $a b$-path through $z$ and $a b \notin E(G)$. Indeed $z$ must be a cut vertex in $G\left[V\left(C_{1}\right) \cup\{a, b\}\right]$ that separates $a$ from $b$ since otherwise an $a b$-path in $G\left[V\left(C_{1}\right) \cup\{a, b\}\right]$ that avoids $z$ could substitute in $H_{w}$ to create a Kuratowski subgraph in $G-f-z$ or $G / f-z$.

Now consider the two components of $G\left[V\left(C_{1}\right) \cup\{a, b\}\right]-z$; call them $U_{a}$ and $U_{b}$, where $a \in V\left(U_{a}\right)$ and $b \in V\left(U_{b}\right)$. Set $U_{a}^{+}=G\left[V\left(U_{a}\right) \cup\{z\}\right]$ and $U_{b}^{+}=G\left[V\left(U_{b}\right) \cup\{z\}\right]$.

Note that $H_{a}$ must be a subgraph of $U_{a}^{+}$or $U_{b}^{+}$, since $H_{a}$ is a subgraph of $G\left[V\left(C_{1}\right) \cup\right.$ $\{a, b\}]$ and $z$ is a cutvertex for this graph. If $H_{a}$ is a subgraph of $U_{a}^{+}$, then $H_{z}$ is also a subgraph of $U_{a}^{+}$since Lemma 4 part (i) shows that $V\left(H_{a} \cap H_{z}\right) \neq \varnothing$. This implies that $\{z, a\}$ is a 2 -cut of $G$ with a heavy component properly contained in $C_{1}$, contradicting that $S$ is basic. So $H_{a}$ is a subgraph of $U_{b}^{+}$. Similarly $H_{b}$ is a subgraph of $U_{a}^{+}$. Lemma 4 part (i) states that $V\left(H_{a} \cap H_{b}\right) \neq \varnothing$, so $V\left(U_{a} \cap U_{b}\right)=\{z\}$ implies $V\left(H_{a} \cap H_{b}\right)=\{z\}$. But recall that $w \in V\left(H_{a} \cap H_{b}\right)$; so $w=z$, a contradiction.

Because $w$ is an apex for $G-f$, for all $f \in E\left(C_{2}^{+}\right)-\{a b\}$, it follows that $E\left(C_{2}^{+}\right)-\{a b\} \subseteq$ $E\left(H_{w}\right)$. Therefore there are no vertices in $V\left(C_{2}\right)$ that have degree two in $H_{w}$ since $\delta(G) \geqslant 3$ would otherwise guarantee an edge in $E\left(C_{2}^{+}\right)-\{a b\}-E\left(H_{w}\right)$. Consequently, all the vertices of $C_{2}$ are branch vertices of $H_{w}$. If $a$ is not a branch vertex of $H_{w}$, then it is a subdividing vertex of $H_{w}$ so there is an edge of $H_{w}$ incident to $a$ whose other endpoint is in $C_{2}$ (because the branch vertices of $H_{w}$ are all in $C_{2} \cup\{a, b\}$ ); call this edge $f$. Now $G / f$ contains $H_{w} / f$, contradicting the claim that established $w$ is an apex for $G / f$. So $a$ is a branch vertex of $H_{w}$. Symmetrically $b$ is a branch vertex of $H_{w}$. Because all of the vertices in $C_{2} \cup\{a, b\}$ are branch vertices of $H_{w}$ and the branch vertices of $H_{w}$ are all in $C_{2} \cup\{a, b\}$, it now follows that $C_{2}^{+}$is isomorphic to $K_{5}, K_{3,3}$, or $K_{3,3}+e$.

The next lemma, a consequence of Lemma 8 and Lemma 9, is a powerful tool to analyze obstructions with more than one 2 -cut.

Lemma 10. Suppose that $G \in \mathcal{F}$ and $\kappa(G)=2$. Every 2-cut of $G$ is basic.

Proof. Suppose that $S=\{a, b\}$ is an arbitrary 2-cut of $G \in \mathcal{F}$. Assume, to the contrary, that $S$ is not basic. Let $C_{S}$ (resp. $L_{S}$ ) denote the heavy (resp. light) component of $G-S$. Because $S$ is not basic, there exists a basic 2-cut $Q$ with heavy (resp. light) component $C_{Q}$ (resp. $\left.L_{Q}\right)$ such that $V\left(C_{Q}\right) \subsetneq V\left(C_{S}\right)$, or equivalently, $\overline{V\left(C_{S}\right)} \subsetneq \overline{V\left(C_{Q}\right)}$. Now

$$
V\left(L_{S}^{+}\right)=V\left(L_{S}\right) \cup S=\overline{V\left(C_{S}\right)} \subsetneq \overline{V\left(C_{Q}\right)}=V\left(L_{Q}\right) \cup Q=V\left(L_{Q}^{+}\right),
$$

so there exists a vertex $z \in V\left(L_{Q}^{+}\right)-V\left(L_{S}^{+}\right)$. Because $Q$ is basic, Lemma 9 implies that $L_{Q}^{+}$is isomorphic to $K_{5}, K_{3,3}$, or $K_{3,3}+e$. But, $V\left(L_{S}^{+}\right) \subsetneq V\left(L_{Q}^{+}\right)$and $z \in V\left(L_{Q}^{+}\right)-V\left(L_{S}^{+}\right)$ means that $L_{S}^{+}$is a subgraph of $K_{5}-z, K_{3,3}-z$, or $K_{3,3}+e-z$. Each of these possibilities contradicts Lemma 8 which shows that $L_{S}^{+}$is non-planar. 


\section{The five types of obstructions and some shared properties}

The connectivity-2 obstructions to the apex family are arranged into five groups. Figure 1 shows a partition of these 133 obstructions according to whether the heavy component of a 2-cut induces a planar graph and further properties of 2-cuts. This partition follows the outlines of our characterization of these graphs.

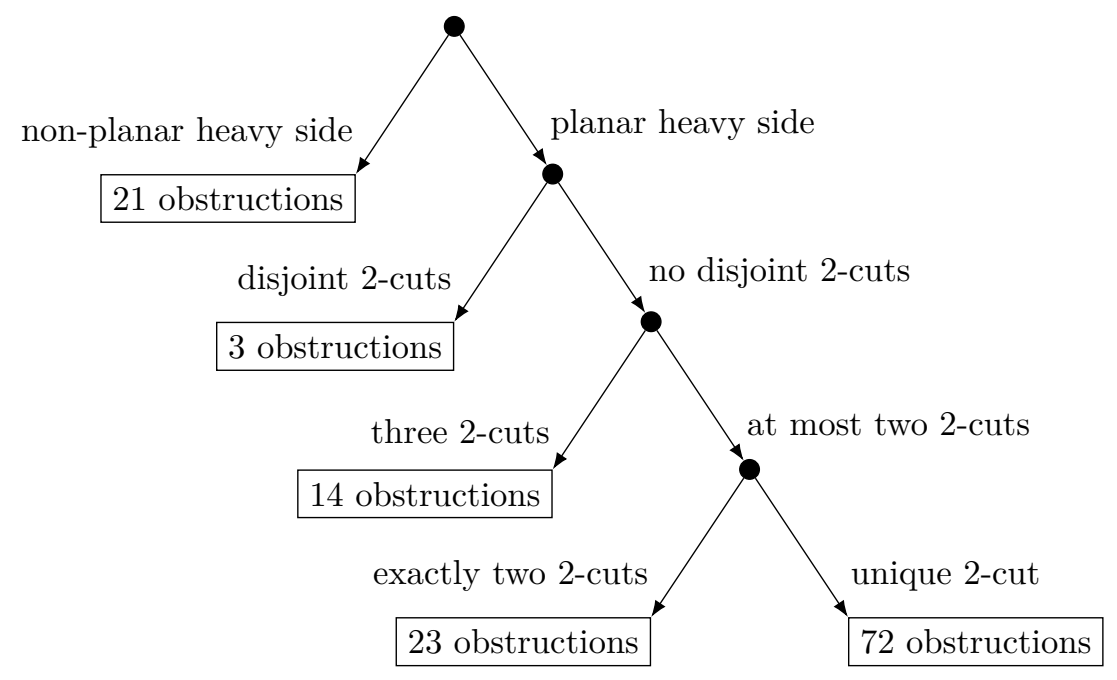

Figure 1: Partition of the 133 connectivity-2 apex obstructions into five types.

Theorem 11. Suppose that $G \in \mathcal{F}, \kappa(G)=2, S=\{a, b\}$ is any 2-cut of $G$, and $C$ is the heavy component of $G-S$. If $H_{a}$ and $H_{b}$ are Kuratowski subgraphs of $G$ avoiding $a$ and $b$, respectively, then

$$
E(C) \subseteq E\left(H_{a} \cup H_{b}\right)
$$

Proof. We argue by contradiction. Assume that $f \in E(C)-E\left(H_{a} \cup H_{b}\right)$. By Lemma 10, $S$ is a basic 2-cut. Lemma 9 guarantees that the augmentation of the light component, $L$, of $G-S$ is isomorphic to $K_{5}, K_{3,3}$, or $K_{3,3}+e$. Now $G-f$ is apex with apex $z$, say. Observe that $z \in V\left(H_{a} \cap H_{b}\right) \subseteq V(G)-(V(L) \cup\{a, b\})$. There can therefore be no $a b$-path in $G[C \cup\{a, b\}]-f-z$ since otherwise the contraction of this path would, together with $L$, realize a Kuratowski subgraph in $G-f-z$. If the endpoints of $f$ are in same component (we may assume the component containing $a$ ) of $G[C \cup\{a, b\}]-z$ (see Figure 2), then, because $H_{z}$ must contain $f$ and $H_{a} \cap H_{z} \neq \varnothing$, both $H_{a}-z$ and $H_{z}$ are also in this component. Consequently the 2-cut $\{a, z\}$ has a heavy component properly contained in $C_{1}$, contradicting that $S$ is basic. 

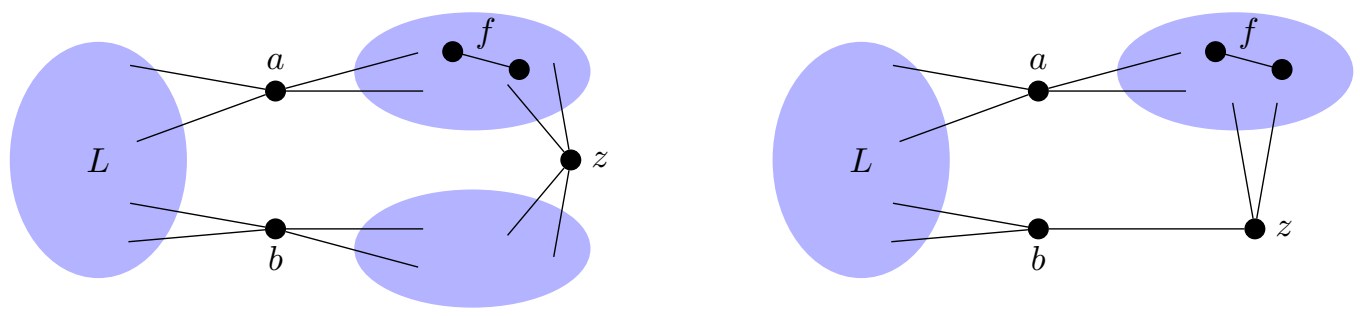

Figure 2: Cases in which endpoints of $f$ are in same component of $G-L-S-z$.

Observe that $f$ is not incident to $z$. So we may assume that the endpoints of $f, u$ and $v$, are in opposite components of $G[C \cup\{a, b\}]-z$. Let us label these components $A$ and $B$, where $A$ (resp. $B$ ) denotes the component containing neighbors of $a$ (resp. $b$ ). Without loss of generality, $u \in A$ and $v \in B$ (see Figure 3).

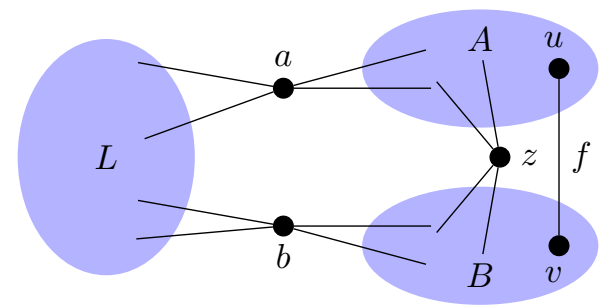

Figure 3: Endpoints of $f$ are in different components of $G-L-S-z$.

Because $f \notin E\left(H_{a} \cup H_{b}\right)$, it follows that $\left|\{u, v\} \cap V\left(H_{a}\right)\right| \leqslant 1$ and $\left|\{u, v\} \cap V\left(H_{b}\right)\right| \leqslant 1$; consequently $H_{a}$ and $H_{b}$ exist in $G / f$, the graph obtained from $G$ by contracting $f$.

Claim: There are two internally vertex-disjoint paths from $a$ to $\{u, z\}$ in $G-b$.

Suppose, to the contrary, that there are not two internally vertex-disjoint paths from $a$ to $\{u, z\}$ in $G-b$. Menger's theorem then guarantees that there is a vertex $w$ in the subgraph of $G$ induced by $A \cup\{a, z\}$ separating $a$ from $\{u, z\}$ (see Figure 4).

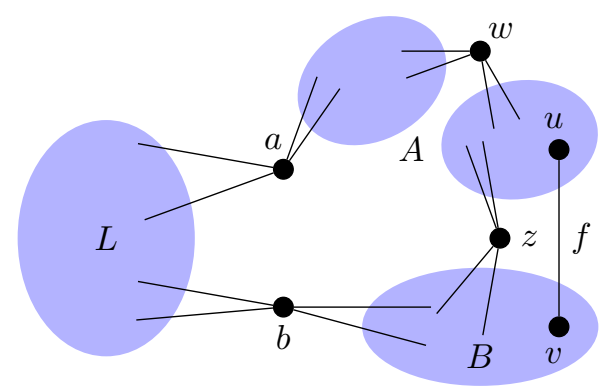

Figure 4: No two internally vertex-disjoint paths implies a vertex $w$.

Recall that $f \in H_{z}$ since $z$ is an apex for $G-f$. Therefore $H_{z}$ in on the $f$-side of the 2-cut $\{b, w\}$. Because $H_{b} \cap H_{z} \neq \varnothing \neq H_{w} \cap H_{z}$, it follows that $H_{b}$ and $H_{w}$ are also on 
the $f$-side of the 2-cut $\{b, w\}$. Consequently the 2-cut $\{b, w\}$ has a heavy component properly contained in $C_{1}$, contradicting that $S$ is basic.

By symmetry, there are two internally vertex-disjoint paths from $b$ to $\{v, z\}$ in $G-a$. This means that there are two internally vertex-disjoint $a b$-paths in $C \cup\{a, b\}$ that remain internally vertex-disjoint after contracting the edge $f$. Now consider $G / f$; it is an apex graph with an apex vertex $z^{\prime}$. As noted earlier, $H_{a}$ and $H_{b}$ exist in $G / f$ so $z^{\prime} \in V\left(H_{a} \cap\right.$ $\left.H_{b}\right) \subseteq V(C)$. But the deletion of the vertex $z^{\prime}$ leaves an $a b$-path on the $C$-side of the 2 -cut $\{a, b\}$ in $G / f$. In particular, this $a b$-path can be contracted to the edge $a b$ which, together with $L$ produces a Kuratowski subgraph in $G / f-z^{\prime}$, contradicting that $z^{\prime}$ is an apex vertex.

\section{The 21 connectivity-2 obstructions with a non-planar heavy component}

We now turn to characterizing apex obstructions in which some 2-cut has a non-planar heavy component.

Theorem 12. Suppose that $G \in \mathcal{F}$ and $\kappa(G)=2$. If some 2-cut of $G$ has a non-planar heavy component, then $G$ is isomorphic to a graph in Figure 5.

Proof. Let $S=\{a, b\}$ be a 2-cut of $G$ with non-planar heavy component $C$ of $G-S$. By Lemma $10, S$ is a basic 2-cut. Lemma 9 guarantees that the augmentation of the light component, $L$, of $G-S$ is isomorphic to $K_{5}, K_{3,3}$, or $K_{3,3}+e$. Observe that $a b \notin E(G)$ since otherwise $G$ contains two disjoint Kuratowski subgraphs, one in $G[V(L) \cup S]$ and one in $C$, contradicting that $G \in \mathcal{F}$ and $\kappa(G)=2$. Similar reasoning shows also that $L^{+} ¥ K_{3,3}+e$, a notable characteristic of this collection of obstructions.

Let $K$ be a minimum order Kuratowski subgraph of $C$. Let $H_{a}=K=H_{b}$ be the two Kuratowski subgraphs avoiding $a$ and $b$. Theorem 11 implies that $E(G) \subseteq$ $E(L) \cup E_{a} \cup E_{b} \cup E(K)$, where recall that $E_{v}=\{e \in E(G): v \in e\}$ denotes the edges of $G$ incident to $v$. This means that $C=K$ and, because $\delta(G) \geqslant 3$, the only possible non-branch vertices of $K$ are neighbors of either $a$ or $b$.

STEP 1: For $v \in S,|N(v) \cap V(K)| \geqslant 2$.

We may assume $v=a$. Suppose, to the contrary, that $|N(a) \cap V(K)| \leqslant 1$. If $|N(a) \cap V(K)|=0$, then $\kappa(G)<2$ so we may assume that $N(a) \cap V(K)=\{w\}$, for some vertex $w \in V(K)$. Now consider the 2-cut $\{b, w\}$ of $G$. Because $H_{w}$ can not be disjoint from $K=H_{b}$, it follows that $H_{w}$ is a subgraph of $G[V(K)-w+b]$. Consequently the 2-cut $\{b, w\}$ has a heavy component that is properly contained in $C_{1}$, contradicting that $S$ is basic.

SteP 2: For $v \in S,|N(v) \cap V(K)| \leqslant 2$. 

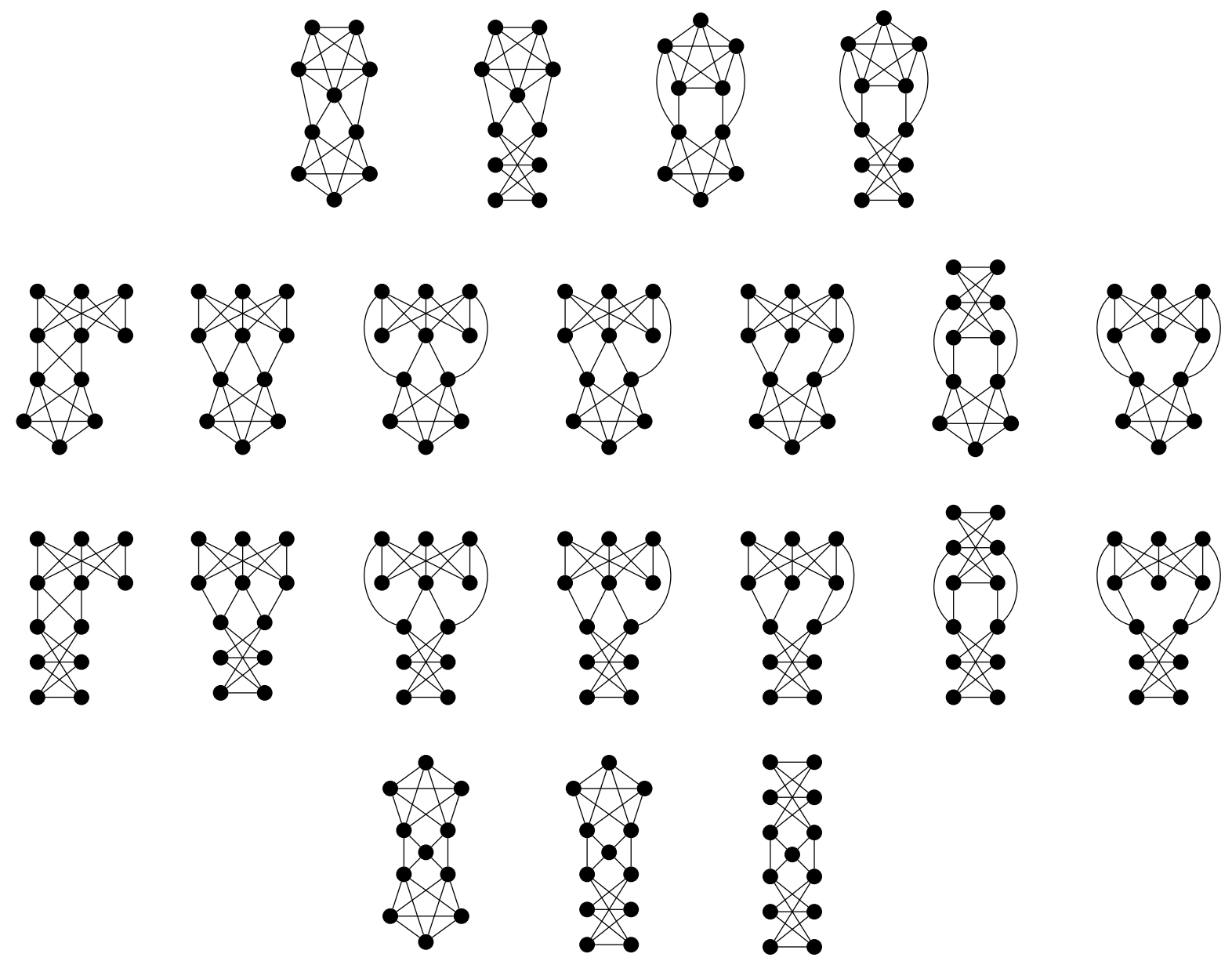

Figure 5: The 21 connectivity-2 apex obstructions with a 2-cut having a non-planar heavy component.

Without loss of generality $v=a$. Suppose, to the contrary, that $|N(a) \cap V(K)| \geqslant 3$. Choose $w \in N(a) \cap V(K)$ and set $e=a w$. Consider $G-e$; it is apex with an apex vertex $z$, say. Now $z \in V(K)$ since $K \subseteq G-e$. Because $|N(a) \cap V(K)| \geqslant 3$, the vertex $a$ has at least one neighbor in $V(K)-\{w, z\}$. By Step 1, the vertex $b$ has at least one neighbor in $G-e-z$. Since $K-z$ is connected, there is an $a b$-path in $G[V(K) \cup\{a, b\}]-e-z$ that produces a Kuratowski subgraph corresponding to the augmentation of $L$ in $G-e-z$, a contradiction.

StEP 3: Any subdividing vertex of $K$ is adjacent to both $a$ and $b$.

Suppose that $w$ is a subdividing vertex of $K$. As noted prior to Step 1, $w \in N(a) \cup N(b)$ since otherwise the minimum degree three of $w$ implies an edge incident to $w$ that is not covered by $K$, contradicting Theorem 11. Assume now, contrary to the claim, that $|N(w) \cap\{a, b\}|=1$. Without loss of generality, aw $\in E(G)$. Choose $w^{\prime} \in$ $(N(w) \cap K)-N(a)$; such a vertex exists since $w$ has two neighbors in $K$ but $a$ only 
has one more neighbor in $K$ besides $w$. Consider $G / w w^{\prime}$; it is apex with apex $z$, say. Note that contracting $w w^{\prime}$ preserves a version of $K$ and both $a$ and $b$ still have two neighbors in this version of $K$ (because $a$ is not adjacent to $w^{\prime}$ and $b$ is not adjacent to $w$ ) implying that there is an $a b$-path in $G[V(K) \cup\{a, b\}] / w w^{\prime}-z$ which determines a Kuratowski subgraph corresponding to the augmentation of $L$ in $G / w w^{\prime}-z$, a contradiction.

STEP 4: $K$ has at most one subdividing vertex.

Observe that, by Steps 1-3, there are at most two subdividing vertices of $K$. Suppose, to the contrary, that $x$ and $y$ are subdividing vertices of $K$. Prior steps guarantee $N(a) \cap V(K)=\{x, y\}=N(b) \cap V(K)$. Let $w \in(V(K) \cap N(x))-y$. Consider these three Kuratowski subgraphs of $G / x w$ : the subgraph induced by $V(L) \cup\{a, b, x\}$, the subgraph induced by $V(L) \cup\{a, b, y\}$, and $K / x w$. These three Kuratowski subgraphs have no common vertex, contradicting that $G / x w$ is apex.

STEP 5: If $K$ has a subdividing vertex $w$, then $N(w) \cap V(K) \subseteq N(a) \cup N(b)$.

Let $x$ and $y$ be the neighbors of $w$ in $K$. If $x \notin N(a) \cup N(b)$, then contracting $w x$ preserves a version of $K$ that must contain an apex $z$ for $G / w x$. However $a$ and $b$ still have two neighbors to this version of $K$ implying that there is a Kuratowski subgraph corresponding to the augmentation of $L$ in $G / w x-z$, a contradiction. Therefore, $x \in N(a) \cup N(b)$. By symmetry, $y \in N(a) \cup N(b)$.

So, in summary, $L^{+}$is isomorphic to $K_{5}$ or $K_{3,3}, C$ is a Kuratowski subgraph, $K$, with at most one subdividing vertex, and $|N(a) \cap V(K)|=2=|N(b) \cap V(K)|$. If $K$ has a subdividing vertex $w$ with $N(w) \cap V(K)=\{x, y\}$, then $x y$ is not an edge of $G$ (by minimality of $K$ ) and, without loss of generality, $N(a) \cap V(K)=\{w, x\}$ and $N(b) \cap V(K)=\{w, y\}$. The reader can now easily verify that the graphs in Figure 5 enumerate the possible apex obstructions with these properties.

\section{The 3 connectivity-2 apex obstructions with planar heavy components and two disjoint 2-cuts}

To complete the characterization of connectivity-2 apex obstructions, it suffices to consider connectivity-2 apex obstructions in which every 2-cut has a planar heavy component. We next consider such connectivity-2 apex obstructions with two disjoint 2-cuts. There are three of these (see Figure 6).

Theorem 13. Suppose $G \in \mathcal{F}, \kappa(G)=2$, and every 2-cut has a planar heavy component. If $G$ has disjoint 2-cuts, then $G$ is isomorphic to a graph in Figure 6.

Proof. Suppose that $S$ and $T$ are disjoint 2-cuts of $G$. Let $S=\{a, b\}$, and let $C_{S}$ be the heavy component of $G-S$. Lemma 10 yields that $S$ and $T$ are basic 2-cuts. Lemma 9 

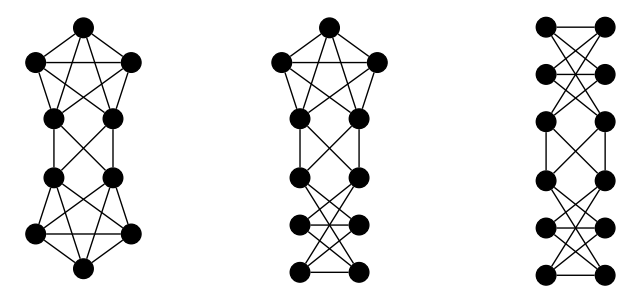

Figure 6: The 3 connectivity-2 apex obstructions in which there are disjoint 2-cuts and every 2 -cut has a planar heavy component.

guarantees that the augmentation, $L_{S}^{+}$, of the light component, $L_{S}$, of $G-S$ is isomorphic to $K_{5}, K_{3,3}$, or $K_{3,3}+e$, each of which is 3 -connected. Similarly $L_{T}^{+}$, the augmentation of the light component of $T$, is isomorphic to $K_{5}, K_{3,3}$, or $K_{3,3}+e$.

CASE 1: $\left|T \cap V\left(L_{S}\right)\right|=2$.

The deletion of $T$ from $L_{S}^{+}$is still connected. It follows that $G-T$ is connected, a contradiction.

CASE 2: $\left|T \cap V\left(L_{S}\right)\right|=1$.

The deletion of a vertex of $T$ from $L_{S}^{+}$is still connected. It follows that $G-T$ can only be disconnected if a vertex of $G$ is a cut vertex of $G$, contradicting that $\kappa(G)=2$.

CASE 3: $\left|T \cap V\left(L_{S}\right)\right|=0$.

Let $T=\{u, v\} \subset C_{S}$ with $L_{T}$ (resp. $C_{T}$ ) the light (resp. heavy) component of $G-T$. Because $G-T$ has two components and one contains $L_{S} \cup S$, it follows that $L_{S} \cup S \subset C_{T}$ because $T$ is basic. Symmetrically, $L_{T} \cup T \subset C_{S}$.

We claim that there exists an av-path in $G-\{b, u\}-V\left(L_{S}\right)-V\left(L_{T}\right)$. It suffices to find an av-path in $G-\{b, u\}$. If $G-\{b, u\}$ is connected, then there is nothing to prove; so assume that $R=\{b, u\}$ is a 2-cut of $G$. Because $S$ is basic, $V\left(C_{R}\right) \cap\left(V\left(L_{S}\right) \cup\{a\}\right) \neq \varnothing$ so $a \in V\left(C_{R}\right)$. Symmetrically, $v \in V\left(C_{R}\right)$. Consequently a path in $C_{R}$ connects $a$ and $v$ in $G-R$.

By symmetry there is an au-path in $G-\{b, v\}-V\left(L_{S}\right)-V\left(L_{T}\right)$, a bv-path in $G-$ $\{a, u\}-V\left(L_{S}\right)-V\left(L_{T}\right)$, and a bu-path in $G-\{a, v\}-V\left(L_{S}\right)-V\left(L_{T}\right)$. All of these paths must be internally vertex disjoint since otherwise there would be an $a b$-path avoiding $\{u, v\}$ (which completes $L_{S}$ to $L_{S}^{+}$in $C_{T}$ ) or a $u v$-path avoiding $\{a, b\}$ (which completes $L_{T}$ to $L_{T}^{+}$in $C_{S}$ ), contradicting that the heavy components of $S$ and $T$ are planar. 


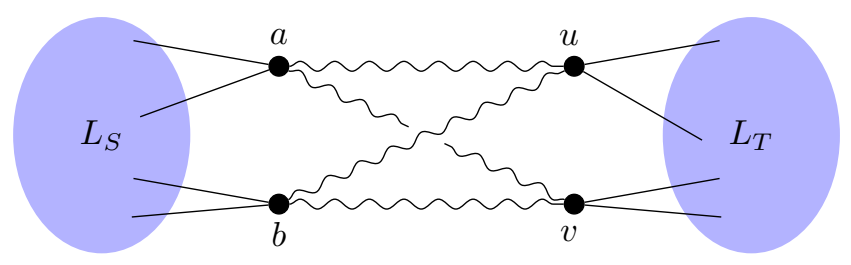

Figure 7: Four internally vertex-disjoint paths connect $\{a, b\}$ to $\{u, v\}$.

Contract all four of these paths into edges connecting $\{a, b\}$ to $\{u, v\}$ (see Figure 7). The graphs in Figure 6 are obstructions and one of them must be obtained via these contractions since $L_{S}^{+}-a b$ and $L_{T}^{+}-u v$ must contain $K_{5}-e$ or $K_{3,3}-e$. Because the original graph is a minor-minimal apex obstruction, it must be one of these.
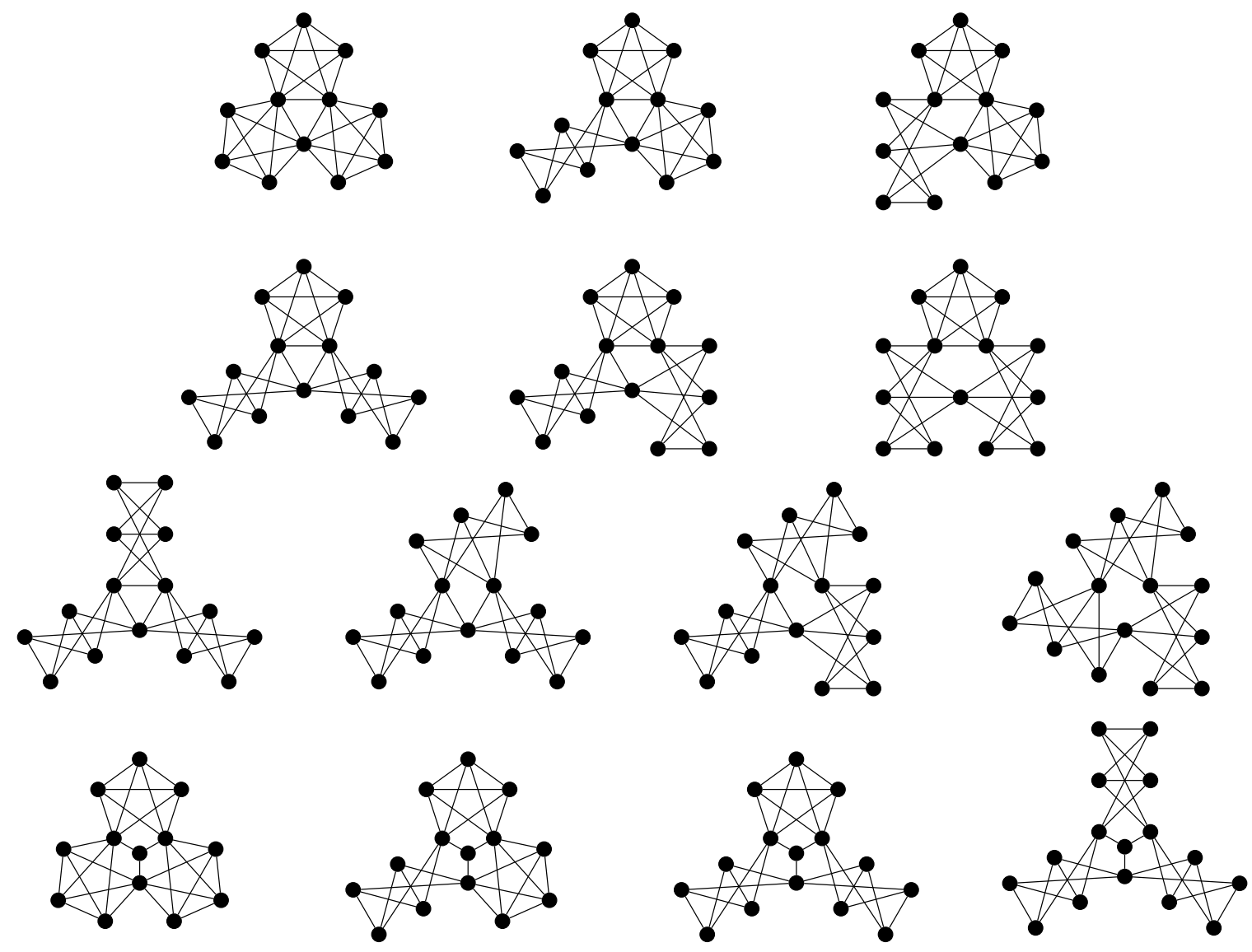

Figure 8: The 14 connectivity-2 apex obstructions with planar heavy components and at least three 2-cuts, but no disjoint 2-cuts. 


\section{The 37 connectivity-2 apex obstructions with planar heavy components and more than one, but no disjoint, 2-cuts}

We next consider the remaining connectivity-2 apex obstructions with with planar heavy components, more than one 2-cut, and every pair of 2-cuts intersect. There are 37 of these obstructions, 14 of which have more than two 2-cuts (see Figure 8) and 23 of which have exactly two 2-cuts (see Figure 11).

Theorem 14. Suppose $G \in \mathcal{F}, \kappa(G)=2$, and every 2-cut has a planar heavy component. If $G$ has more than one 2 -cut and every pair of 2-cuts intersect, then $G$ is isomorphic to a graph in Figure 8 or Figure 11.

Proof. Suppose $S=\{a, b\}$ and $T=\{a, x\}$ are 2-cuts of $G$. By Lemma 10, all 2-cuts of $G$ are basic. Let $C_{S}$ and $C_{T}$ be the heavy components of $G-S$ and $G-T$, respectively; let $L_{S}$ and $L_{T}$ be the corresponding light components. Lemma 9 guarantees that $L_{S}^{+}$and $L_{T}^{+}$are isomorphic to $K_{5}, K_{3,3}$, or $K_{3,3}+e$. If $x$ were a vertex in $L_{S}$ then $G-\{a, x\}$ would be connected because $L_{S}^{+}$is 3-connected; so $x \notin V\left(L_{S}\right)$. Symmetrically $b \notin V\left(L_{T}\right)$. Because $G-T$ has two components and one component contains $L_{S} \cup\{b\}$, it follows that $L_{S} \cup\{b\} \subset C_{T}$ because $T$ is basic. Symmetrically, $L_{T} \cup\{x\} \subset C_{S}$. Consequently $L_{S} \cap L_{T}=\varnothing$.

CASE 1: $R=\{b, x\}$ is a 2 -cut of $G$.

Let $L_{R}$ denote the light component of $G-R$. Reasoning as in the paragraphs above, we find that $L_{R}^{+}$is isomorphic to $K_{5}, K_{3,3}$, or $K_{3,3}+e$, and $L_{R} \cap L_{S}=\varnothing=L_{R} \cap L_{T}$. Consider $H_{a}, H_{b}$, and $H_{x}$. Note $b \in V\left(H_{a}\right)$ and $a \in V\left(H_{b}\right)$ because $C_{S}$ is planar. Symmetrically $a \in V\left(H_{x}\right)$ and $x \in V\left(H_{a}\right)$ because $C_{T}$ is planar; and $b \in V\left(H_{x}\right)$ and $x \in V\left(H_{b}\right)$ because $C_{R}$ is planar. In this case, $H_{x} \cap L_{T}=\varnothing=H_{x} \cap L_{R}$ because $\{a, b\} \subset V\left(H_{x}\right)$. A symmetric statement applies to $H_{a}$ and $H_{b}$.

Now let $P=V(G)-V\left(L_{S}\right)-V\left(L_{T}\right)-V\left(L_{R}\right)-\{a, b, x\}$.

CASE 1A: $P=\varnothing$.

Because $H_{x} \cap L_{T}=\varnothing=H_{x} \cap L_{R}$ and $P=\varnothing$, we find $V\left(H_{x}\right) \subset V\left(L_{S}\right) \cup S$. This implies $G\left[V\left(L_{S}\right) \cup S\right] \cong L_{S}^{+}$. It follows that $G\left[V\left(L_{S}\right) \cup S\right]$ is isomorphic to one of $K_{5}, K_{3,3}$, or $K_{3,3}+e$. Symmetrically $G\left[V\left(L_{T}\right) \cup T\right]$ and $G\left[V\left(L_{R}\right) \cup R\right]$ are also isomorphic to one of these graphs. There are ten non-isomorphic graphs that result from these possibilities, and they each produce an obstruction, as shown in the top ten graphs of Figure 8.

CASe 1B: $P \neq \varnothing$.

Let $W$ be a component of $P$. Observe that $W$ must have vertices adjacent to all three vertices $a, b$, and $x$ since otherwise the removal from $G$ of one of the 2 -cuts $R, S$, or $T$ would have more than two components, contradicting Lemma 7 . Now 
consider the graph $H$ obtained from $G$ by contracting $W$ to a vertex $w$. The vertex $w$ has neighborhood $\{a, b, x\}$ in $H$.

If $a b, a x, b x \in E(G)$, then $H-w$ (hence $G-W$ ) has a minor isomorphic to one top ten graphs in Figure 8, a contradiction. Indeed if any one these three edges is in $E(G)$, then such a minor can be produced: for example, if $a b \in E(G)$, then contract $H / w x$. So we may assume $a b, a x, b x \notin E(G)$. We claim that $H\left[V\left(L_{S}\right) \cup S\right]$ is isomorphic to $K_{5}-e$ or $K_{3,3}-e$. It suffices to prove that $H\left[V\left(L_{S}\right) \cup S\right]+a b \neq K_{3,3}+e$. If $H\left[V\left(L_{S}\right) \cup S\right]+a b \cong K_{3,3}+e$, then the contraction $H / w x$ again contains a minor isomorphic to one top ten graphs in Figure 8, a contradiction.

By symmetry we conclude that $H\left[L_{S} \cup S\right], H\left[L_{T} \cup T\right]$ and $H\left[L_{R} \cup R\right]$ are all isomorphic to $K_{5}-e$ or $K_{3,3}-e$. There are four possible graphs constructed using such components and they are shown at the bottom of Figure 8. Because these graphs are obstructions, it follows that $H$ must be one of these graphs (and $P=W=\{w\}) ;$ and $G \cong H$.

CASE 2: $R=\{b, x\}$ is not a 2 -cut of $G$.

As in Case $1, b \in V\left(H_{a}\right)$ and $a \in V\left(H_{b}\right)$ since otherwise $C_{S}$ is non-planar. Also $x \in$ $V\left(H_{a}\right)$. Now let $P=V(G)-V\left(L_{S}\right)-V\left(L_{T}\right)-\{a, b, x\}$. Note that $V\left(H_{a}\right) \cap V\left(L_{T}\right)=\varnothing$ since $\{b, x\} \subset V\left(H_{a}\right)$ and $\{a, x\}$ separates $b$ from $L_{T}$. Similarly, $V\left(H_{a}\right) \cap V\left(L_{S}\right)=\varnothing$. Consequently $V\left(H_{a}\right) \subseteq V(P) \cup\{b, x\}$ and $P \neq \varnothing$.

Every component of $G[P]$ has a neighbor of $a$; otherwise $\{b, x\}$ is a 2-cut (which is Case 1). So, there exists an edge $e=a w$ with $w$ in $P$.

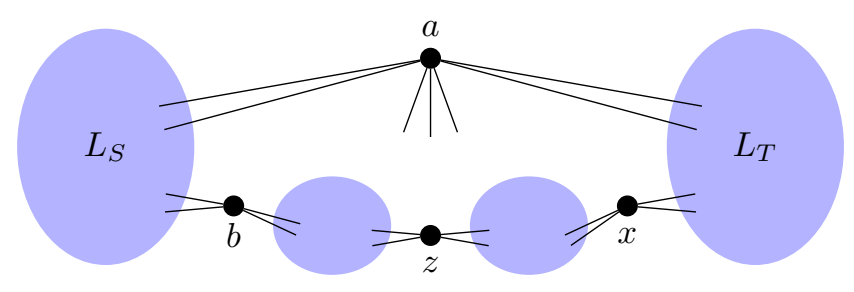

Figure 9: Augmentations of $L_{S}$ and $L_{T}$ to Kuratowski subgraphs.

We claim that $w \in V\left(H_{a}\right)$. If $w \notin V\left(H_{a}\right)$, then $G / e$ must have an apex, say $v$, such that $v \in V\left(H_{a}\right) \subset P \cup\{b, x\}$. Note that $v \notin\{b, x\}$ because $G / e-b$ contains an augmentation of $L_{T}$ with ax-path through $P-b$, and similarly $G / e-x$ contains an augmentation of $L_{S}$ with $a b$-path through $P-x$. If $v \in P$ then $v$ would separate $b$ from $x$ in $H_{a}$ otherwise $L_{S}$ can be completed to a Kuratowski subgraph in $G / e-v$ with a $v$-avoiding $b x$-path in $G[P \cup\{b, x\}]$ continuing to $a$ through $L_{T}$. But $H_{a}$ is 2-connected, so $v$ can not separate $b$ from $x$ in $H_{a}$. So $G / e$ has no apex, a contradiction.

Next we claim that $N(a) \cap(P \cup\{b, x\})=\{w\}$. Suppose, on the contrary, there is a vertex $v \in P \cup\{b, x\}$ such that $v \neq w$ and $f=a v \in E(G)$. Consider an apex $u$ for $G-f$. Notice that $u \in V\left(H_{a}\right) \subseteq P \cup\{b, x\}$. Now $u \neq x$ because a Kuratowski 
subgraph exists by augmenting $G\left[V\left(L_{S}\right) \cup S\right]$ with an $a b$-path from $a$ to $w$ through $P$ to $b$. Similarly, $u \neq b$; so $u \in P$. Because $u \notin\{b, x\}, u$ must separate $b$ from $x$ in $G-f$; otherwise $L_{S}$ can be completed to a Kuratowski subgraph in $G-f-u$ with a $u$-avoiding $b x$-path continuing to $a$ through $L_{T}$. However, $b$ and $x$ are in $H_{a}$ which is a subgraph $G[P \cup\{b, x\}]$. Because $H_{a}$ is 2-connected, $u$ can not separate $b$ from $x$, a contradiction. So $N(a) \cap(P \cup\{b, x\})=\{w\}$ (see Figure 10).

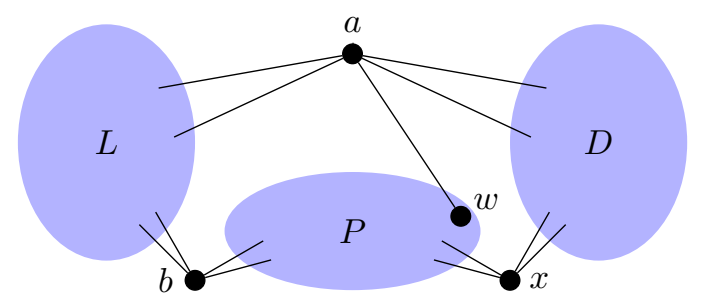

Figure 10: $N(a) \cap(P \cup\{b, x\})=\{w\}$.

Similar reasoning as the last paragraph shows that every edge of $G[P \cup\{b, x\}]$ must be an edge of $H_{a}$; otherwise deleting this edge produces a graph with no apex.

Now suppose that there is an edge $f \in E\left(H_{a}\right)$ such that $|f \cap\{b, w, x\}| \leqslant 1$ and $f$ is incident to a non-branch vertex of $H_{a}$. Consider $G / f$; it contains $b, w, x$. As before, $b$ and $x$ cannot be apex vertices for $G / f$. Furthermore, no vertex in $V\left(H_{a}\right)$ separates $b$ from $x$, so they can not be apex vertices. Hence $G / f$ has no apex, a contradiction. We conclude that every edge of $H_{a}$ has branch vertices of $H_{a}$ as endpoints or its endpoints are both in $\{b, w, x\}$.

We claim that every vertex of $P \cup\{b, x\}$ is a branch vertex of $H_{a}$. Because every edge of $G[P \cup\{b, x\}]$ is an edge of $H_{a}$ and every edge of $H_{a}$ has branch vertices of $H_{a}$ as endpoints or its endpoints are both in $\{b, w, x\}$, it suffices to prove that $b, w$, and $x$ are branch vertices of $H_{a}$.

If $b$ is not a branch vertex of $H_{a}$, then the two neighbors of $b$ in $H_{b}$ must be $w$ and $x$. Because every edge of $G[P \cup\{b, x\}]$ is an edge of $H_{a}$, it follows that $b$ has only $w$ and $x$ as neighbors in $G[P \cup\{b, x\}]$. This implies $\{w, x\}$ is a 2-cut of $G$ separating $P-\{w\}$ from $b$; this 2-cut is disjoint from $\{a, b\}$, contradicting that $G$ has no disjoint 2-cuts. Hence $b$ must be a branch vertex of $H_{a}$. By symmetry, $x$ is also a branch vertex of $H_{a}$.

Apply similar reasoning to show $w$ is a branch vertex. If $w$ is not a branch vertex of $H_{a}$, then the two neighbor of $w$ in $H_{a}$ (and hence in $G[P \cup\{b, x\}]$ ) are $b$ and $x$. This implies that $\{b, x\}$ is a 2 -cut of $G$, (which is Case 1 ).

So every vertex of $P \cup\{b, x\}$ is a branch vertex of $H_{a}$. This means that there are 23 possible graphs shown in Figure 11. The three vertices in the center of these figures are, clockwise from the top of the three vertices, $a, x, b$. The light component, $L_{S}$, appears to the left, and $L_{T}$ appears to the right. The unsubdivided Kuratowski subgraph $H_{a}$ appear at the bottom of each graph drawing. 

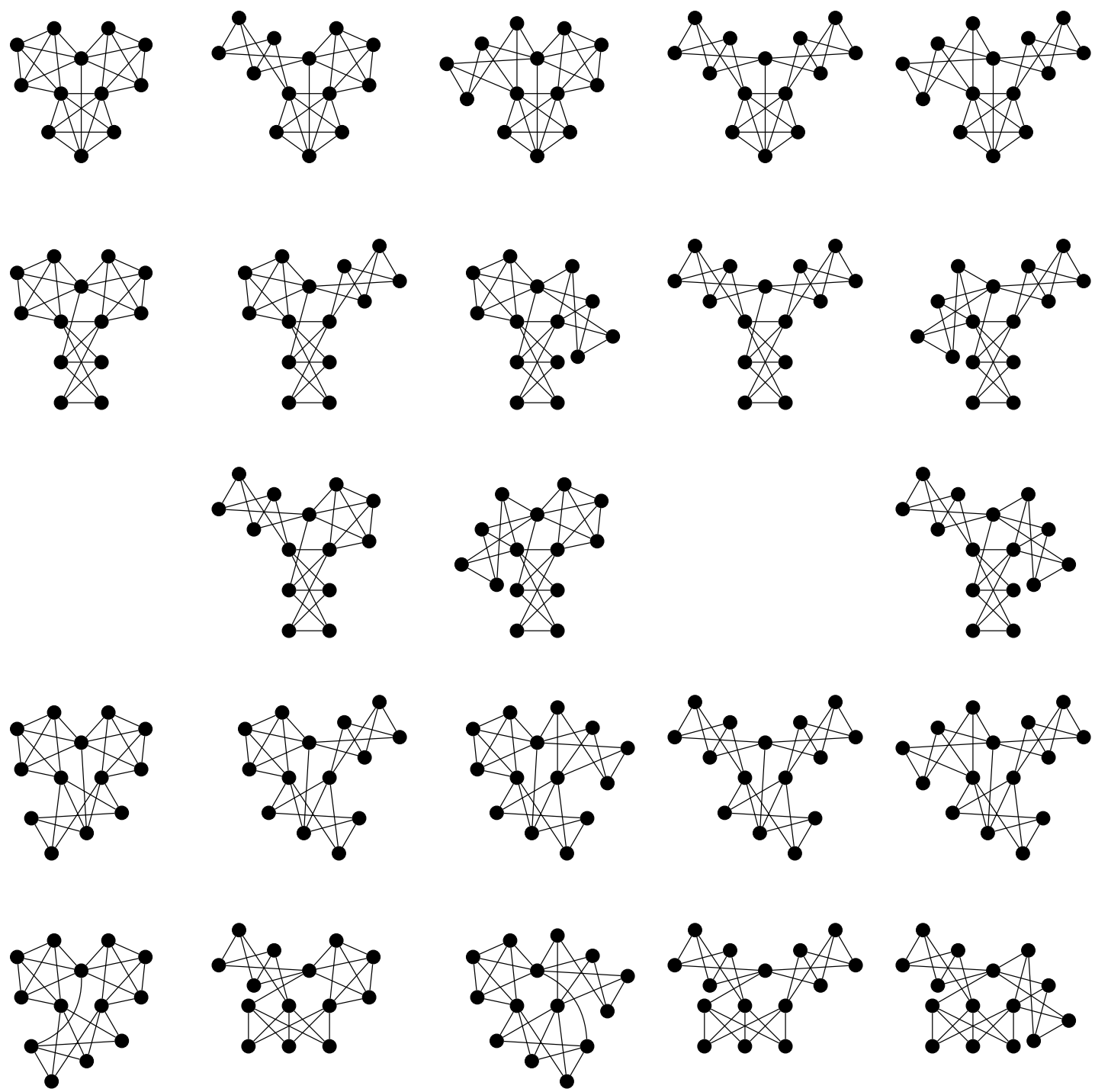

Figure 11: The 23 connectivity-2 apex obstructions with planar heavy components and exactly two 2 -cuts that intersect.

\section{The 72 connectivity-2 apex obstructions with a unique 2-cut having a planar heavy component}

We next consider the final collection of connectivity-2 apex obstructions, the obstructions with a unique 2-cut whose removal produces a planar heavy component. These obstructions are considerably more difficult to characterize than the prior types, so we must present several structural results before tackling the final characterization.

In this section we let $C$ denote the heavy component of an apex obstruction after deleting the unique 2-cut, with $C$ representing either this subgraph or its vertex set, as 
convenient. Similar expedient recastings also apply to other subgraphs and their vertex sets, with context alleviating any ambiguities.

There are 72 apex obstructions determined in this section. Thirty-three of these obstructions (see Figure 61) have a heavy component $C$ and a unique 2-cut $\{a, b\}$ such that $G[C \cup\{a, b\}]$ has a 2-cut separating $a$ from $b$; the remaining thirty-nine (see Figure 62) have no such 2-cut.

The critical intermediate goal is to prove that there exist two Kuratowski subgraphs whose branch vertices cover every vertex of the unique 2-cut and every vertex of the heavy component (Theorem 25). Adding the light-component Kuratowski subgraph to these two makes three Kuratowski subgraphs whose branch vertices cover the entire vertex set of the apex obstruction, a property that reduces the problem of characterizing all of these remaining obstructions to a very small number of cases which we complete in the final subsection.

Many preliminary lemmas are needed for this final analysis. To simplify the presentation, we adopt the following notation and assumptions in this section.

Assumptions 15. Standard assumptions for this section:

- $G$ is a minor-minimal non-apex graph.

- $G$ has connectivity two and a unique 2-cut $S=\{a, b\}$.

- $G-S$ has two components, the heavy component $C$ and the light component $L$.

- $C$ is a planar graph.

\subsection{Some preliminary lemmas for the final case}

This subsection presents several lemmas establishing basic structure.

Lemma 16. Under Assumptions 15:

(i) If $H_{a}$ and $H_{b}$ are any Kuratowski subgraphs of $G$ avoiding a and $b$, respectively, then $b \in V\left(H_{a}\right)$ and $a \in V\left(H_{b}\right)$.

(ii) $a b \notin E(G)$.

(iii) If $H_{a}$ and $H_{b}$ are any Kuratowski subgraphs of $G$ avoiding a and $b$, respectively, then

$$
E(G[C \cup\{a, b\}]) \subseteq E\left(H_{a} \cup H_{b}\right) .
$$

(iv) Any 2-cut of $G[C \cup\{a, b\}]$ that separates a from $b$ does not induce an edge.

Proof. (i) Because $C$ is planar, any Kuratowski subgraph of $G[C \cup\{a, b\}]$ must contain at least one vertex from $\{a, b\}$. Consequently any Kuratowski subgraph avoiding $a$ must contain $b$ and vice versa.

(ii) If $e=a b$ were an edge of $G$, then $G-e$ would have an apex $z \in V\left(H_{a}\right) \cap V\left(H_{b}\right) \subseteq C$. Indeed $z$ would have to separate $a$ from $b$ in $C$; otherwise an $a b$-path in $G-e$ could complete $L$ to $L^{+}$. Consequently, either $\{a, z\}$ or $\{b, z\}$ would be another 2-cut in $G$. 
(iii) By Theorem 11, $E(C) \subseteq E\left(H_{a}\right) \cup E\left(H_{b}\right)$. Since (i) states that $a b \notin E(G)$, to prove (iii) it suffices (by symmetry of $a$ and $b$ ) to prove that every edge $a w$ with $w \in V(C)$ is also an edge in $H_{a}$ or $H_{b}$. We argue by contradiction.

Assume that there is an edge $f=a w$, with $w \in V(C)$ such that $f$ is not in $E\left(H_{a}\right) \cup$ $E\left(H_{b}\right)$. Consider $G-f$; it has an apex vertex $z$. Note that $H_{a}$ and $H_{b}$ are still intact in $G-f$, so $z \in V\left(H_{a}\right) \cap V\left(H_{b}\right) \subset C$; in particular, $z \notin\{a, b\}$. Now $z$ must separate $a$ from $b$ in $G[C \cup\{a, b\}]-f$, otherwise an $a b$-path avoiding $z$ and $f$ could be used to augment $L$ to a Kuratowski subgraph $L^{+}$, contradicting that $G-f-z$ is planar. Define $A$ to be the set of vertices that are connected to $a$ via a $z$-avoiding path in $G[C \cup\{a, b\}]-f$. Because $a \in V\left(H_{b}\right)$ it has at least two neighbors in $H_{b}$; at least one of these is not $z$. Consequently, $A \neq \varnothing$. Now observe that $\{a, z\}$ is 2 -cut of $G$ separating $b$ from vertices in $A$, contradicting that $G$ has a unique 2-cut.

(iv) Assume, to the contrary, that a 2-cut, $\{w, x\}$ of $G[C \cup\{a, b\}]$ separates $a$ from $b$ and $f=w x \in E(G)$. Partition the vertices of $C-\{w, x\}$ into two sets:

$$
A=\{v \in C-\{w, x\} \text { : there is a va-path avoiding }\{w, x\} \text { in } G[C \cup\{w, x, a\}]\}
$$

and

$$
B=\{v \in C-\{w, x\} \text { : there is a } v b \text {-path avoiding }\{w, x\} \text { in } G[C \cup\{w, x, b\}]\}
$$

(see Figure 12).

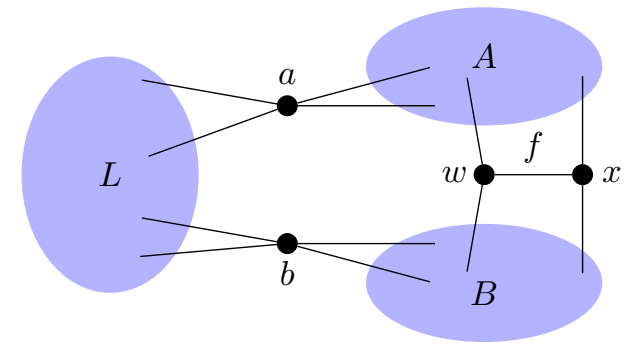

Figure 12: $V\left(H_{b}\right) \subseteq A \cup\{a, w, x\}$ and $V\left(H_{a}\right) \subseteq B \cup\{b, w, x\}$

Let $H_{a}$ and $H_{b}$ be minimum-sized Kuratowski subgraphs of $G$ avoiding $a$ and $b$, respectively. Because $V\left(H_{b}\right) \subset C \cup\{a\}$ and the branch vertices of $H_{b}$ must all be on one side of $\{w, x\}$, it follows from $w x \in E(G)$ and the minimum size of $H_{b}$ that $V\left(H_{b}\right) \subseteq A \cup\{a, w, x\}$. A similar argument shows $V\left(H_{a}\right) \subseteq B \cup\{b, w, x\}$. In particular, $A \neq \varnothing \neq B$.

Observe that $w$ must have neighbors in $A$ and $B$ since $\{a, x\}$ and $\{b, x\}$ are not 2cuts of $G$; similarly $x$ must have neighbors in $A$ and $B$. Note too that there must be a $w x$-path in $G[A \cup\{w, x\}]-f$; otherwise $\{a, w\}$ or $\{a, x\}$ would be another 2-cut of $G$. Symmetrically there must be a $w x$-path in $G[B \cup\{w, x\}]-f$. Finally there must be two vertex-disjoint paths from $a$ to $\{w, x\}$ in $G[A \cup\{a, w, x\}]$ since $\{a, b\}$ is the only 2-cut of $G$. Similarly, there must be two vertex-disjoint paths from $b$ to $\{w, x\}$ in $G[B \cup\{b, w, x\}]$.

Now we claim that $G-f$ has no apex. Suppose, to the contrary, that $y$ is an apex for $G-f$. If $y \in A \cup\{w, x\}$, then there remains an $a$ to $\{w, x\}$-path that can be completed 
to an $a b$-path in $G[C \cup\{a, b\}]-f-y$, thus augmenting $L$ to $L^{+}$in $G-f-y$. A similar argument applies if $y \in B \cup\{w, x\}$. So it remains to consider $y \in\{a, b\} \cup L$. If $y \in\{a\} \cup L$, then consider $H_{a}$ in $G-f-y$. Recall that $V\left(H_{a}\right) \subseteq B \cup\{b, w, x\}$, so $H_{a}$ is only possibly missing the edge $f$ in $G-f-y$. Now a $w x$-path whose internal vertices are entirely in $A$ can substitute for the edge $f$ in $H_{a}$. Hence no vertex in $\{a, b\} \cup L$ is an apex for $G-f$. A similar argument show that $y=b$ is not an apex in $G-f$. Hence $G-f$ has no apex, a contradiction.

The next lemma embodies fundamental arguments to which we shall often appeal. The lemma may be interpreted as stating that $J=G[C \cup\{a, b\}]$ is a minor-minimal double apex graph with roots $a$ and $b$ (see Theorem 27).

Lemma 17. Assume Assumptions 15 and $J=G[C \cup\{a, b\}]$. For any edge $e \in E(J)$,

(i) $J-e-a$ is planar or $J-e-b$ is planar (or both), and

(ii) $J / e-a$ is planar or $J / e-b$ is planar (or both).

In part (ii) the notation means that if e is incident to vertex a (resp. b) in J, then e is contracted in $\mathrm{J} / \mathrm{e}$ to form a new vertex also labeled a (resp. b).

Proof. (i) Assume, to the contrary, that $J-e-a$ and $J-e-b$ are both non-planar. Consider Kuratowski subgraphs $H_{a}$ and $H_{b}$ in $J-e$ avoiding $a$ and $b$, respectively. Now observe that $e \notin E\left(H_{a}\right) \cup E\left(H_{b}\right)$, contradicting Lemma 16 part (iii).

(ii) Assume, to the contrary, that $J / e-a$ and $J / e-b$ are both non-planar. Consider $G / e$; it has an apex $z$. Because $J / e-a$ and $J / e-b$ are both non-planar, $z \notin\{a, b\}$. Now $z$ must separate $a$ from $b$ in $J / e$, otherwise a $z$-avoiding path in $G / e$ could complete $L$ to $L^{+}$, contradicting that $z$ is an apex for $G / e$. Because $\{a, z\}$ and $\{b, z\}$ are not 2-cuts of $G$, it follows that $z$ is the contracted vertex created by contracting the edge $e$. Consequently the vertices in $e$ form a 2 -cut in $J$ separating $a$ from $b$, contradicting Lemma 16 part (iv).

The next lemma gives information about edges shared by Kuratowski subgraphs missing $a$ and $b$.

Lemma 18. Assume Assumptions 15. Suppose $H_{a}$ and $H_{b}$ are any Kuratowski subgraphs avoiding $a$ and $b$ respectively. If $u v=e \in E\left(H_{a}\right) \cap E\left(H_{b}\right)$, then

(i) $u$ and $v$ are both branch vertices of $H_{a}$, or

(ii) $u$ and $v$ are both branch vertices of $H_{b}$,

or both.

Proof. Let $J=G[C \cup\{a, b\}]$. If $u$ is a branch vertex of neither $H_{a}$ nor $H_{b}$, then $H_{a}$ and $H_{b}$ remain non-planar after contracting $e$. Consequently $J / e-a$ and $J / e-b$ are both non-planar, contradicting Lemma 17 part (ii). So $u$ is a branch vertex of $H_{a}$ or $H_{b}$; similarly $v$ is a branch vertex of $H_{a}$ or $H_{b}$. If $u$ or $v$ is a branch vertex of both, then 
(i) or (ii) follows. So we may assume, to the contrary, that $u$ is a branch vertex of $H_{a}$ but not a branch vertex of $H_{b}$ and, symmetrically, $v$ is a branch vertex of $H_{b}$ but not a branch vertex of $H_{a}$. In this case, again $H_{a}$ and $H_{b}$ remain non-planar after contracting $e$ so $J / e-a$ and $J / e-b$ are both non-planar, again contradicting Lemma 17 part (ii).

The next lemma give a powerful tool to prove that vertices are branch vertices of Kuratowski subgraphs avoiding $a$ and $b$.

Lemma 19. Assume Assumptions 15. Suppose $H_{a}$ and $H_{b}$ are any Kuratowski subgraphs avoiding $a$ and $b$ respectively.

(i) If $v \in V\left(H_{a}\right)-\left(V\left(H_{b}\right) \cup\{a, b\}\right)$, then $v$ and all of its neighbors are branch vertices of $H_{a}$.

(ii) If $v \in V\left(H_{b}\right)-\left(V\left(H_{a}\right) \cup\{a, b\}\right)$, then $v$ and all of its neighbors are branch vertices of $H_{b}$.

Proof. By symmetry it suffices to prove (i). Let $v \in V\left(H_{a}\right)-\left(V\left(H_{b}\right) \cup\{a, b\}\right)$. Assume, to the contrary, that $w$ is $v$ or a neighbor of $v$ but $w$ is not a branch vertex of $H_{a}$. If $w=v$, then because $w$ has only degree two in $H_{a}$, there is an edge incident to $w$ (since $\delta(G) \geqslant 3$, Lemma 1 ) that is not in $E\left(H_{a}\right) \cup E\left(H_{b}\right)$, contradicting Lemma 16 part (iii). So $w \neq v$. Let $e=w v$ and $J=G[C \cup\{a, b\}]$.

Because $w$ is not branch of $H_{a}$, the Kuratowski $H_{a}$ survives in $J / e$. Also, $H_{b}$ survives in $J / e$ because $v \notin V\left(H_{b}\right)$. Consequently $J / e-a$ and $J / e-b$ are non-planar, contradicting Lemma 17 part (ii).

\subsection{Vertices in the unique 2-cut must be branch vertices}

The main goal in this subsection is to prove that vertex $a$ must be a branch vertex of any Kuratowski subgraph avoiding $b$ and, symmetrically, $b$ must be a branch vertex of any Kuratowski subgraph avoiding $a$ (statement of Corollary 22). To simplify statements in this subsection we focus just on proving the latter claim since the former claim follows from symmetry. So the reader should keep in mind the symmetrical consequences of the results that follow.

First we focus on the connectivity of $C$.

Lemma 20. Assume Assumptions 15 and $H_{a}$ is any Kuratowski subgraph avoiding a. If $b$ is not a branch vertex of $H_{a}$, then $C$ is 2-connected.

Proof. Let $H_{b}$ be any Kuratowski subgraph missing $b$. If $b$ is not a branch vertex of $H_{a}$, then it has exactly two neighbors in $H_{a}$. Since all edges of $G[C \cup\{a, b\}]$ must be covered by $E\left(H_{a}\right) \cup E\left(H_{b}\right)$ (Lemma 16 part (iii)), it follows that $N(b) \cap C=\{x, y\}$, for some $x, y \in C$. Suppose, to the contrary, that $c$ is a cut vertex of $C$. If $c=x, c=y$, or $x$ and $y$ are in the same component of $C-c$, then $\{a, c\}$ is another 2-cut of $G$, a contradiction. Therefore $c$ separates $x$ from $y$ in $C$. In $G[C \cup\{b\}]$, the set $\{b, c\}$ is a 2-cut so all branch vertices of $H_{a}$ must be on one side of this 2-cut. If one side of this 2-cut contains no branch 
vertices of either $H_{a}$ or $H_{b}$, then (since each side is non-empty) there is an edge $e$ incident to $c$ that can be contracted that preserves $H_{a}$ and $H_{b}$. Consequently, $G[C \cup\{a, b\}] / e-a$ and $G[C \cup\{a, b\}] / e-b$ are non-planar, contradicting Lemma 17 part (ii). So, each side of $G[C \cup\{b\}]-c$ has branch vertices. That is, the branch of $H_{a}$ are all in one side, and the branch vertices of $H_{b}$, except possibly $a$, are on the other side. Consequently, the vertex $a$ can have at most one neighbor, call it $z$, in one of the sides, which without loss of generality, contains $y$. If there is a $c z$-path avoiding $y$, then $b y$ is an edge that can be contracted preserving $H_{a}$ and $H_{b}$; that is, $G[C \cup\{a, b\}] / b y-a$ and $G[C \cup\{a, b\}] / b y-b$ are non-planar, contradicting Lemma 17 part (ii). So every $c z$-path contains $y$. If $y=z$, then $\{c, y\}$ or $\{b, y\}$ is another 2-cut of $G$. If $y \neq z$, then $\{a, y\}$ is a 2-cut separating $c$ from $z$.

Now we are ready to prove a claim that plays a major role in the final characterization of the connectivity-2 apex obstructions. The claim states that, under the Assumptions 15, the vertices in the unique 2-cut, $a$ and $b$, must be branch vertices of all of their Kuratowski witnesses. This is the statement of Corollary 22, which will be a consequence of Proposition 21. Though Proposition 21 proves a seemingly weaker existential claim, its significance is indicated by its long proof in which several 3-connected apex obstructions play a vital role, including the Petersen-family graphs $M, Y^{-}, P_{7}$ and the Petersen graph itself.

Proposition 21. If Assumptions 15 are satisfied, then there exists a Kuratowski subgraph avoiding vertex $a$ in which $b$ is a branch vertex.

Proof. Suppose, to the contrary, that $b$ is not a branch vertex of any Kuratowski subgraph avoiding $a$. Let $H_{a}$ be a Kuratowski subgraph of $G$ avoiding $a$. Necessarily $b \in V\left(H_{a}\right)$ (Lemma 16 part (i)), so $b$ has at least two neighbors in $H_{a}$. If $\left|N_{G}(b) \cap C\right|>2$, then some edge in $G[C \cup\{b\}]$ incident to $b$ does not appear in $H_{a}$ or any Kuratowski subgraph of $G$ avoiding $b$, contradicting Lemma 16 part (iii). Consequently we may assume $\left|N_{G}(b) \cap C\right|=$ 2 ; say $N_{G}(b) \cap C=\{x, y\}$.

Fix a plane embedding of $C$. Let $K=V\left(H_{a}\right)-b$. The plane embedding of $C$ includes a plane embedding of $G[K]$. Because $H_{a}-b$ is a subdivision of $K_{5}-e$ or $K_{3,3}-e$, a plane embedding of $H_{a}-b$ is unique; consequently, a plane embedding of $G[K]$ is unique. Define $A$ to be the component of $G[C \cup\{a\}-K]$ that contains the vertex $a$, and let $\bar{a}$ be the vertex obtained by contracting $A$ in this graph to a single vertex.

Clearly $G[C \cup\{a\}]$ is not planar. It is possible that this graph becomes planar after contracting $A$ to $\bar{a}$. We focus on $H_{a}-b$, a subgraph of $G[C \cup\{a\}]$. If it is possible to append $\bar{a}$ (and its incident edges to neighbors in $H_{a}-b$ ) to the plane embedding of $H_{a}-b$ without introducing a crossing in the plane (i.e. extend the plane embedding of $H_{a}-b$ to a plane embedding of $H_{a}-b+\bar{a}$ ), then we say that $\bar{a}$ hits only one face; otherwise $\bar{a}$ hits multiple faces.

The following steps provide contradictions that complete the proof of the proposition.

STEP 1: For any $H_{a}, \bar{a}$ hits only one face of the plane embedding of $H_{a}-b$. 
Suppose, on the contrary, there is a choice of $H_{a}$ such that $\bar{a}$ hits multiple faces of the plane embedding of $H_{a}-b$. There are two cases according to whether $H_{a}-b$ is a subdivision of $K_{5}-e$ or $K_{3,3}-e$.

Consider first the case in which $H_{a}-b$ is a subdivision of $K_{5}-e$. The planar embedding of this subdivision is unique; it is shown in Figure 13.

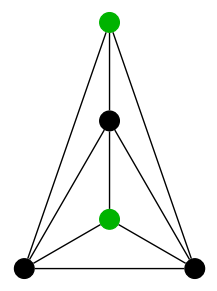

Figure 13: The case when $H_{a}-b$ is a subdivision of $K_{5}-e$; neighbors of $b$ are green.

Lemma 20 implies that $\bar{a}$ must have at least two neighbors in $H_{a}-b$. Suppose that $\bar{a}$ has only two such neighbors. There are seven non-isomorphic ways (see Figure 14) that $\bar{a}$ has exactly two neighbors in this subdivision of $K_{5}-e$ so that the resulting graph is not planar.
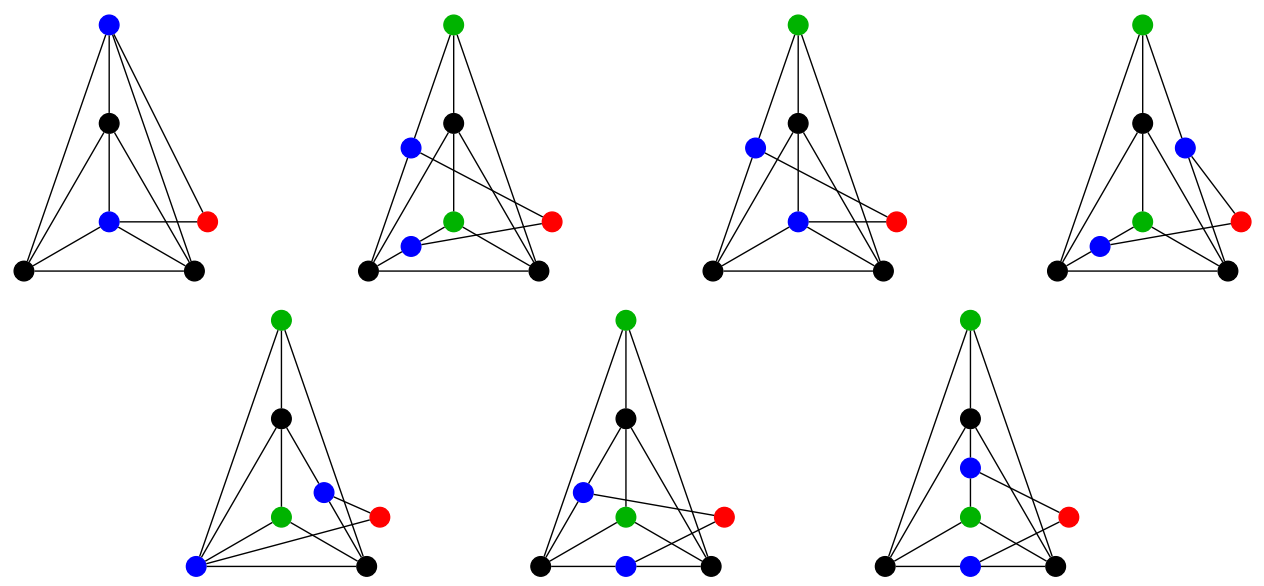

Figure 14: In the case $H_{a}-b$ is a subdivision of $K_{5}-e$, there are seven ways that $\bar{a}$ has two neighbors in $H_{a}-b$ not both of which are in the same face.

The three right-most cases on the top row of Figure 14 all include neighbors of $\bar{a}$ (shown in blue) that can be contracted along edges of $H_{a}-b$ so that one is contracted to $x$ and the other is contracted to $y$. These contractions produce a proper minor of $J=G[C \cup\{a, b\}]$ in which the contracted $H_{a}-b$, still a subdivision of $K_{5}-e$, extends to a subdivision of $K_{5}$ by adding either $b$ or $\bar{a}$. This contradicts Lemma 17 part (ii), so these cases cannot occur.

The left-most case on the top row of Figure 14 implies that $\{x, y\}$ is another 2-cut of $G$, a contradiction. As an aside, this last configuration actually has disjoint 2-cuts, $\{x, y\}$ and $\{a, b\}$, showing that this case would produce obstructions shown in Figure 6. 
The cases shown on the bottom row of Figure 14 can be dismissed as follows. Consider adding the vertex $b$ to each graph along with the edge $\bar{a} b$ (which corresponds to contracting $L$ in $G$ to the edge $a b$ ). Figure 15 shows the resulting graphs (where now vertex $b$ is shown in red). The leftmost graph of Figure 15 is a 3-connected apex obstruction from the Petersen family; it is usually called $M$. Because $M$ cannot appear as a proper minor of $G$, this case does not occur. The other two graphs of Figure 15 contain $M$ as a proper minor, so those cases too cannot occur.
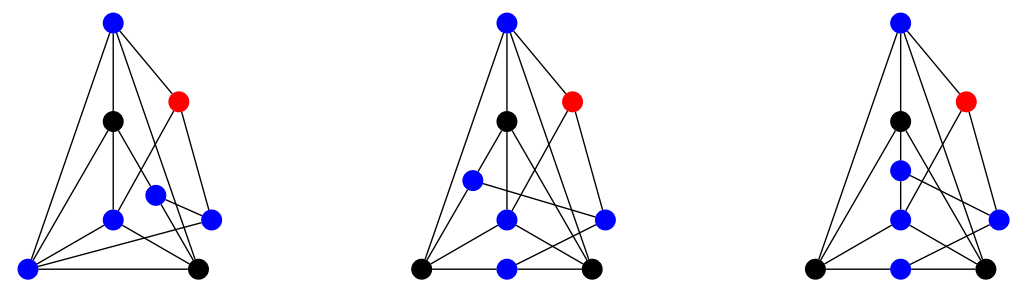

Figure 15: The leftmost graph is $M$, a Petersen family apex obstruction. The other two graphs properly contain $M$ as a minor. The vertex $b$ is shown in red.

To complete the analysis of the case in which $H_{a}-b$ is a subdivision of $K_{5}-e$, suppose now that $\bar{a}$ has at least three neighbors in $H_{a}-b$. Observe that $\bar{a}$ cannot have two neighbors that attach as in Figure 14 since each of these cases produces a contradiction to Lemma 17 (parts (i) and (ii)) or a minor of $M$. Consequently, we may assume that the three neighbors of $\bar{a}$ are not all on one face, but any two of them are on a single face. There are four non-isomorphic ways to select such neighbors for $\bar{a}$ (see Figure 16).
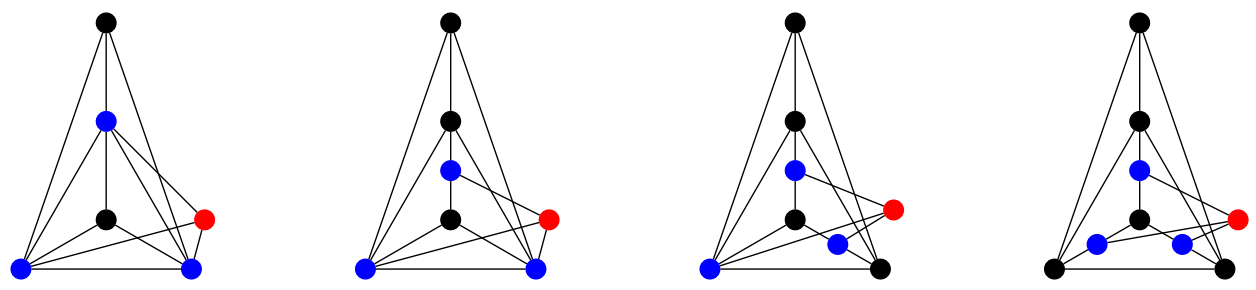

Figure 16: The four non-isomorphic ways that $\bar{a}$ (shown in red) has three neighbors (shown in blue) in $H_{a}-b$ (which is a subdivision of $K_{5}-e$ ) so that all three neighbors are not on the same face, but any two are on a single face.

One way is to choose three neighbors of $\bar{a}$ to be the three degree four vertices of $H_{a}-b$. The remaining three cases can be contracted to this case. Now adding to this graph the vertex $b$ and the edge $\bar{a} b$, as before, produces the graph shown in Figure 17. It is an apex obstruction from the Petersen family; it is called $Y^{-}$. It cannot occur as a minor of $G$, so this completes the analysis of Step 1 when $H_{a}-b$ is a subdivision of $K_{5}-e$ 


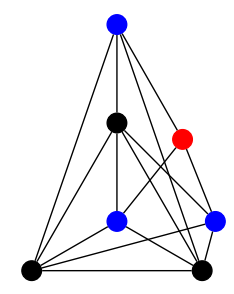

Figure 17: The graph $Y^{-}$, a Petersen family apex obstruction. Vertex $b$ is shown in red.

Consider next the case in which $H_{a}-b$ is a subdivision of $K_{3,3}-e$. The planar embedding of this subdivision is unique; it is shown in Figure 18.

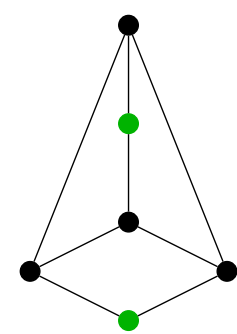

Figure 18: The case when $H_{a}-b$ is a subdivision of $K_{3,3}-e$; neighbors of $b$ are green.

The analysis now follows along similar reasoning as the case in which $H_{a}-b$ is a subdivision of $K_{5}-e$. Suppose that $\bar{a}$ has only two neighbors in $H_{a}-b$. There are four non-isomorphic ways (see Figure 19) that $\bar{a}$ has exactly two neighbors in this subdivision of $K_{3,3}-e$ so that the resulting graph is not planar.
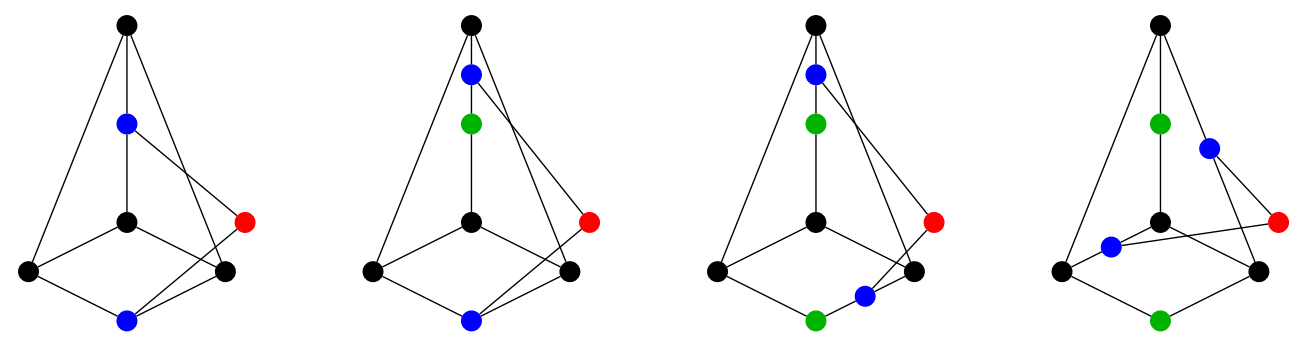

Figure 19: In the case $H_{a}-b$ is a subdivision of $K_{3,3}-e$, there are four ways that $\bar{a}$ has two neighbors in $H_{a}-b$ not both of which are in the same face.

In the left three graphs of Figure 19 one can reason, as before in the $K_{5}-e$ case, that either $\{x, y\}$ is another 2-cut of $G$ or one neighbor of $\bar{a}$ can be contracted to $x$ and the other to $y$ so that a contradiction to Lemma 17 part (ii) occurs. So $\bar{a}$ has no two neighbors of this type, leaving only the exclusion of the graph at the right of Figure 19. This graph extends with $b$ and the edge $\bar{a} b$ to the graph shown in Figure 20. This graph is isomorphic to the Petersen graph, an apex obstruction, so cannot be a proper minor of $G$. 


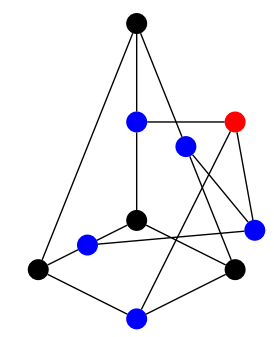

Figure 20: In the case $H_{a}-b$ is a subdivision of $K_{3,3}-e$, a graph isomorphic to the Petersen graph emerges. Vertex $b$ is red.

So it remains to consider the case in which $\bar{a}$ has three (or more) neighbors in $H_{a}-b$ such that, not all are in a single face, but any two of them are on a single face. There are ten non-isomorphic ways $\bar{a}$ has three such neighbors (see Figure 21).
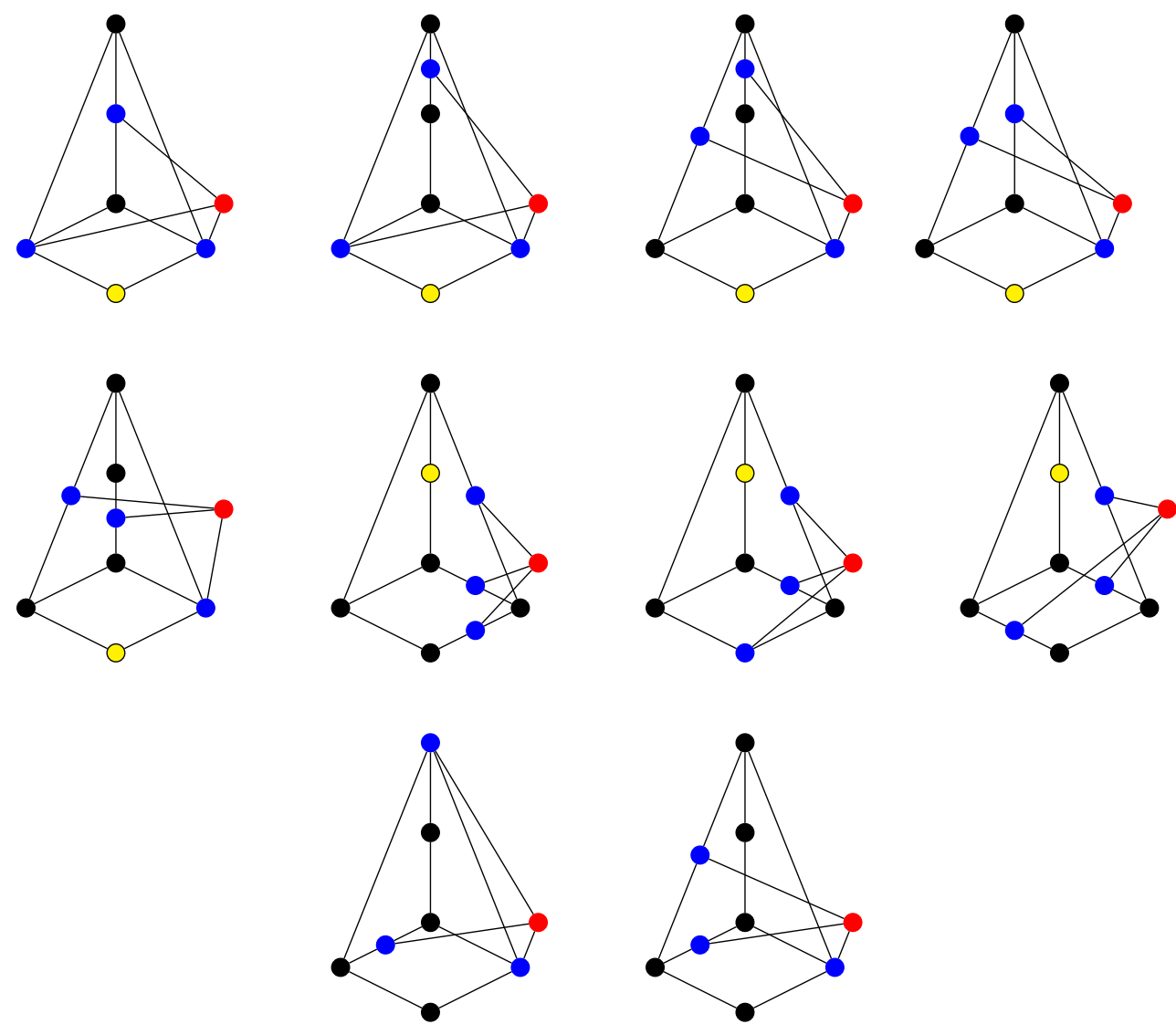

Figure 21: The ten non-isomorphic ways $\bar{a}$ (shown in red) has three neighbors in $H_{a}-b$ (which is a subdivision of $K_{3,3}-e$ ) so that all three neighbors (shown in blue) are not on the same face, but any two are on a single face.

In the eight graphs in the top two rows of Figure 21 a vertex, either $x$ or $y$, can be deleted and the remaining graph remain non-planar; in each case the deletable vertex is 
shown in yellow. Consequently in these graphs either $b x$ or $b y$, according to whether $x$ or $y$ is deletable, is an edge that contradicts Lemma 17 (part (ii)). So these eight graphs cannot occur.

The bottom right graph of Figure 21 can be contracted to the bottom left graph, so it remains to dismiss the bottom left graph. As before, add vertex $b$ and the edge $\bar{a} b$ to this graph produces the graph shown in Figure 22. This graph is a graph isomorphic to the graph $P_{7}$, a Petersen-family minor. Because it is an apex obstruction it cannot occur as a proper minor of $G$. This completes the proof of Step 1.

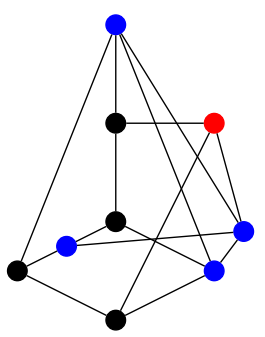

Figure 22: In the case $H_{a}-b$ is a subdivision of $K_{3,3}-e$, a graph isomorphic to the graph $P_{7}$, a Petersen family apex obstruction, emerges. Vertex $b$ is red.

By Step 1, we may assume that $\bar{a}$ has neighbors in only one face of the plane embedding of $H_{a}-b$. Let $F$ be a face of the plane embedding of $H_{a}-b$ such that $\bar{a}$ has only neighbors in it. There could be two such faces, a technicality addressed later (see Step 5). Without loss of generality, the face $F$ is a face not incident to $y$ (see Figure 23). Recall that $K=V\left(H_{a}\right)-b$ and $A$ is the component of $G[C \cup\{a\}-K]$ that contains the vertex $a$. Let $\operatorname{int}(F)$ denote all of the vertices of $C-K$ that appear in the interior of $F$. More precisely, $\operatorname{int}(F)$ consists of the vertices in the interior of the region of the plane avoiding $y$ that is bounded by $F$ in our fixed plane embedding of $C$.

Choose $H_{a}$ that minimizes the number of vertices that are on the boundary or the interior of $F$. We may also assume that embedding has been fixed to minimize the number of crossings produced when $a$ is reinserted into the face $F$.
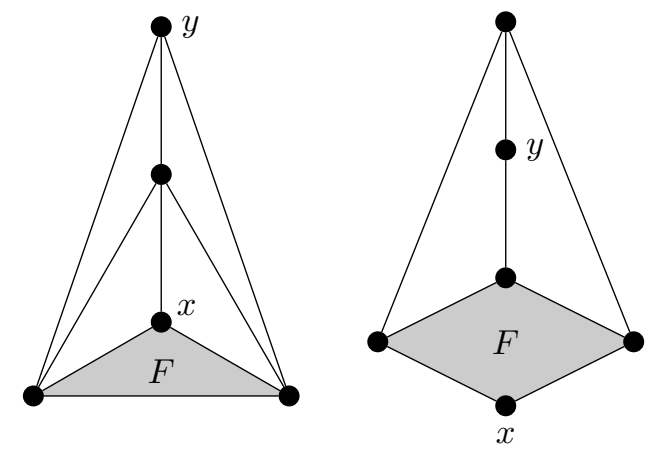

Figure 23: Whether $H_{a}-b$ is a subdivision of $K_{5}-e$ or $K_{3,3}-e$, we may assume $F$ is a face that is not incident to $y$ in the unique plane embedding of $H_{a}-b$. 
Define $C_{1}, \ldots, C_{t}$ to be the components of $G[C \cup\{a\}-K]$ that intersect $\operatorname{int}(F) \cup\{a\}$. Note that $A$ is one of these components; we assume $C_{1}=A$. Observe that $\left|N\left(C_{i}\right) \cap F\right| \geqslant 3$, for $i>1$; otherwise there would be another 2-cut in $G$. This argument does not apply to the component $C_{1}$ because it contains the vertex $a$ which has neighbors in $L$. However, Lemma 20 implies that $\bar{a}$ must have at least two neighbors in $H_{a}-b$; hence $C_{1}$ must have at least two neighbors on $F$.

Define $S$ to be the set of vertices on $F$ with at least one neighbor in $\operatorname{int}(F) \cup\{a\}$. Note that $|S| \geqslant 2$ since if $S=\{v\}$, then $\{b, v\}$ is another 2-cut of $G$. An important observation is that, by Lemma 19, all the vertices in $\operatorname{int}(F)$ and their neighbors on $F$ are all branch vertices of $H_{b}$. So all vertices in $S$ are branch vertices of $H_{b}$, except possibly vertices whose only neighbor in $\operatorname{int}(F) \cup\{a\}$ is $a$.

STEP 2: $\left|C_{i}\right|=1$, for all $i=1, \ldots, t$.

Assume, to the contrary, that $\left|C_{i}\right|>1$ for some $i=1, \ldots, t$. Let $u \in C_{i}-\{a\}$ and $v \in C_{i}$ be chosen so that $u v$ is an edge. Let $e=u v$. Lemma 19 implies that all of the vertices in $C_{i}$ and the neighbors of $u$ must be branch vertices of $H_{b}$.

Recall that $J=G[C \cup\{a, b\}]$. It suffices to show that there exists a Kuratowski minor in $G[C \cup\{a\}]$ avoiding $y$, since then $y b$ can be contracted so that $J / y b-a$ and $J / y b-b$ are both non-planar, contradicting Lemma 17 part (ii). Consider $J / e$ and let $w$ be the composite vertex in $J / e$ that results from identifying $u$ and $v$. Because $H_{a}$ is untouched by the contraction of $e$, Lemma 17 part (ii) implies that $J / e-b$ is planar. Because $H_{a}-b$ has a unique planar embedding and it is a subgraph of $J / e-b$, the cycle $F$ must separate $y$ from $w$ in any plane embedding of $J / e-b$. In particular, the faces containing $w$ do not contain $y$. Let $W$ be the plane graph formed by the union of the faces containing $w$ in a plane embedding of $J / e-b$. Now it suffices to find a Kuratowski minor in the graph obtained from $W$ by splitting $w$ back into $u$ and $v$.

CASE 1: $u$ and $v$ have a common neighbor.

Let $w_{1}$ be the common neighbor of $u$ and $v$. Because $w_{1}$ is also a branch vertex of $H_{b}$ and all edges of $C$ are covered by $E\left(H_{a}\right) \cup E\left(H_{b}\right)$, it follows that all edges of the triangle $G\left[\left\{u, v, w_{1}\right\}\right]$ are in $H_{b}$; so $H_{b}$ is a subdivision of $K_{5}$. In this case, $u$ and $v$ must have degree exactly four in $G[C \cup\{a\}]$. Suppose $N(u)=\left\{v, w_{1}, w_{2}, w_{3}\right\}$. Note that $u$ and its four neighbors, are the five branch vertices of $H_{b}$.

Suppose that $\left\{w_{1}, w_{2}, w_{3}\right\} \subset N_{J}(v)$. This implies $N_{J}(u)-\{v\}=N_{J}(v)-\{u\}=$ $\left\{w_{1}, w_{2}, w_{3}\right\}$. Splitting $w$ back into $u$ and $v$ in $W$ produces a $K_{5}$ subdivision in $G[C \cup\{a\}]$ avoiding $y$ (see Figure 24 .

If $\left\{w_{2}, w_{3}\right\} \not \subset N(v)$, then $v=a$ (because $v \neq a$ implies that all of $v$ 's neighbors are branch vertices). Without loss of generality, $w_{3}$ is not a neighbor of $v$.

The vertices $w_{1}, w_{2}$, and $w_{3}$ partition the cycle $W-w$ into into three segments. Splitting $w$ back into $u$ and $v$ must produce a crossing since $G[C \cup\{a\}]$ is non-planar. In particular, splitting $w$ back into $u$ and $v$ produces a Kuratowski subdivision or minor in $G[C \cup\{a\}]$ avoiding $y$ in each of the remaining cases:

(i) $v$ has a neighbor in the segment between $w_{2}$ and $w_{3}\left(K_{3,3}\right.$ subdivision), 

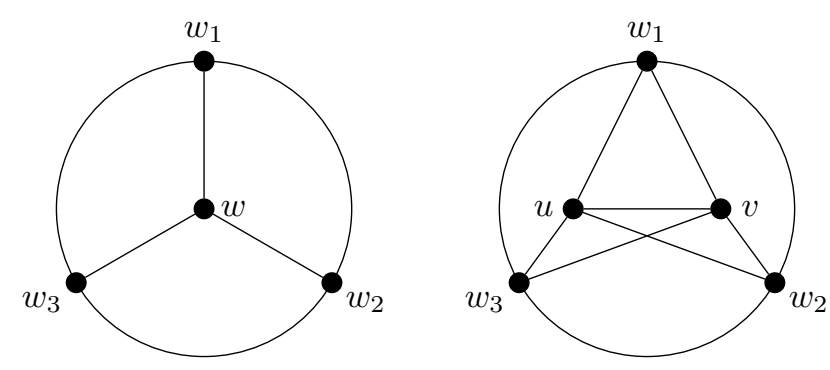

Figure 24: The faces of the plane embedding of $J / e-b$ containing $w$ on left. On the right, a subdivision of $K_{5}$ is found in $J-\{b, y\}$ if $\left\{w_{1}, w_{2}, w_{3}\right\} \subset N(u) \cap N(v)$.
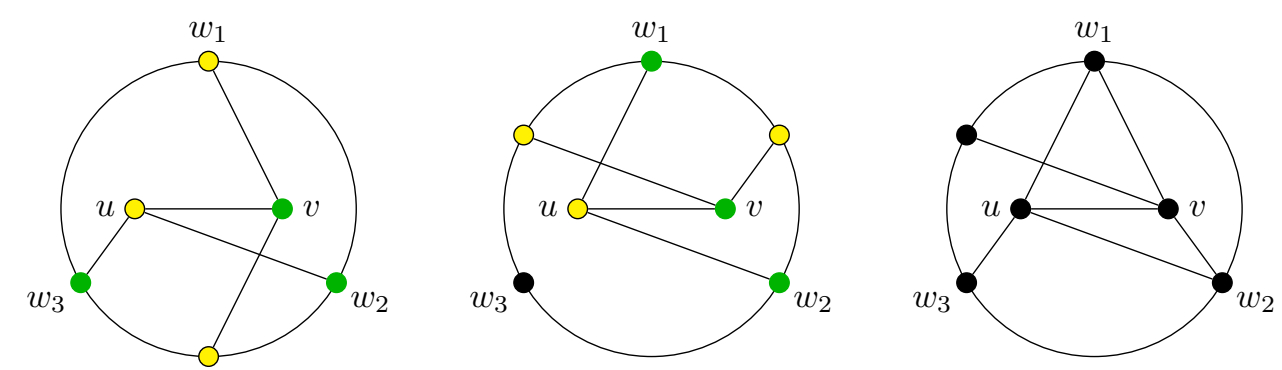

Figure 25: Splitting $w$ back into $u$ and $v$ produces a Kuratowski minor in $G[C \cup\{a\}]$ avoiding $y$. Cases (i), (ii), and (iii) in this order from left to right.

(ii) $v$ has neighbors in the segments between $w_{1}$ and $w_{3}$ and between $w_{2}$ and $w_{3}\left(K_{3,3}\right.$ subdivision), and

(iii) $v$ is adjacent to $w_{1}$ and $w_{2}$ so has a neighbor between $w_{1}$ and $w_{3}$ ( $K_{5}$ minor).

See Figure 25 which gives drawings for cases 1), 2), and 3) in this order from left to right.

CASE 2: $u$ and $v$ have no common neighbor.

Suppose first that $v \neq a$. Under this supposition, all of the neighbors of $v$ are also branch vertices of $H_{b}$ (Lemma 19). Also the degree of $u$ and $v$ are at least three, so $u$ and $v$ have four different neighbors implying that $H_{b}$ is a subdivision of $K_{3,3}$. It follows that $u$ and $v$ both have degree three in $G[C \cup\{a\}]$ and have no common neighbors. Set $N(u)-\{v\}=\{\alpha, \beta\}$ and $N(v)-\{u\}=\{\gamma, \delta\}$. In $W$ the neighbors of $w$ (the four vertices $\alpha, \beta, \gamma, \delta)$ must appear along the face created by deleting $w$ so that the $\alpha$ and $\beta$ are not consecutive (see left of Figure 26), otherwise the plane embedding of $J / e-b$ could be extended to $J-b$, contradicting that $G[C \cup\{a\}]$ is non-planar. This produces a subdivision of $K_{3,3}$ that does not contain $y$ (see right of Figure 26).

So $v=a$. We may assume that, except for $v$, all of the neighbors of $u$ are on $F$ (otherwise we could substitute one of them for $v$ instead).

If $C_{1}$ contains three vertices, say $C_{1}=\{u, v, z\}$ then, because $u \neq a \neq z$, the closed neighborhoods of $u$ and $z$ are all branch vertices of $H_{b}$ (Lemma 19). Because all of the neighbors of $u$ are on $F, u$ and $z$ are not adjacent so $H_{b}$ is a subdivision of $K_{3,3}$. All edges of $C$ are covered by $E\left(H_{a}\right) \cup E\left(H_{b}\right)$, so $u$ and $z$ must have exactly three neighbors. Let $w_{1}$ and $w_{2}$ be the two neighbors of $u$ on $F$. This implies $N_{J}(u)=\left\{v, w_{1}, w_{2}\right\}=N_{J}(z)$. Let 

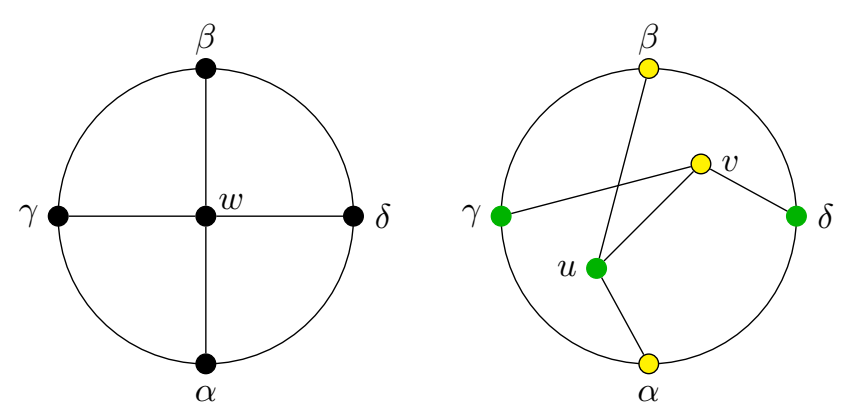

Figure 26: The faces of the plane embedding of $J / e-b$ containing $w$ on left. On the right, a subdivision of $K_{3,3}$ is found in $J-\{b, y\}$.

$\alpha$ be a neighbor of $v$ on $F$. A subdivision of $K_{3,3}$ exists in $G[F \cup\{u, v, z\}]$ (with branch vertices $\left.u, v, z, w_{1}, w_{2}, \alpha\right)$. Because this subdivision of $K_{3,3}$ avoids $y$, we may assume that $C_{1}=\{u, v\}$.

If $H_{b}$ is a subdivision of $K_{5}$, then $u$ has three neighbors $w_{1}, w_{2}$, and $w_{3}$ on $F$. Because $u$ and $v$ have no common neighbors and the neighbors of $v$ must be on $F$, at least two of the neighbors of $v$ must be in $F$ in different interior faces, otherwise this planar embedding of $C$ could be extended to a planar embedding of $G[C \cup\{a\}]$. This implies a $K_{3,3}$ subdivision avoiding $y$, a contradiction (see left and center panel of Figure 25).

If $H_{b}$ is a subdivision of $K_{3,3}$, then $u$ and $v$ have degree three but no common neighbors in $G[C \cup\{a\}]$. In this case $u$ has two neighbors $w_{1}, w_{2}$ on $F$. The two neighbors of $v$ on $F$ must appear in different interior faces, otherwise this planar embedding of $C$ could be extended to a planar embedding of $G[C \cup\{a\}]$. This implies a $K_{3,3}$ subdivision avoiding $y$, a contradiction (see center panel of Figure 25).

\section{STEP 3: $t \leqslant 2$.}

We shall prove Step 3 by contradiction: assume that $t>2$. Step 2 implies $C_{2}$ and $C_{3}$ each contain exactly one vertex. These two vertices are nonadjacent branch vertex of $H_{b}$. The neighbors $C_{2}$ and $C_{3}$ on $F$ must be branch vertices of $H_{b}$ too. So $H_{b}$ is necessarily a subdivision of $K_{3,3} ; C_{1}$ and $C_{2}$ have the same three neighbors in $S$. Consequently, the graph $G\left[F \cup C_{2} \cup C_{3}\right]$ contains a subdivision of $K_{5}-e$ that has an embedding in the plane (inherited from the planar embedding of $C$ ) in which $F$ contains the degree-four branch vertices of the subdivided $K_{5}-e$ and the other branch vertices are in $\operatorname{int}(F)$. This is a contradiction because $K_{5}-e$ has a unique planar embedding in which the triangle connecting the degree-four vertices is a Jordan curve separating the degree-three vertices. So this proves Step 3 and we may assume that $t \leqslant 2$.

\section{STEP 4: $t \leqslant 1$.}

For the rest of the proof we may assume, by Step 2, that $C_{1}=\{a\}$. Assume, to the contrary, that $t=2$. Let $w$ denote the one vertex in $C_{2}$. Note that all of the vertices in the closed neighborhood of $w$ are branch vertices of $H_{b}$ (by Lemma 19) and, $d_{G}(w)=3$ or $d_{G}(w)=4$ (because $E(C) \subset E\left(H_{a}\right) \cup E\left(H_{b}\right)$ by Lemma 16 part (iii)). 
Recall that $K=V\left(H_{a}\right)-b$. An edge in $G[K]$ that is not used by the subdivision $H_{a}$ is called a chord. To the given fixed, plane embedding of $C$ add the vertex $a$ (and its incident edges), placing $a$ inside $F$ in general position so as to minimize the number of crossings. The next definition of crossing chords and related arguments are now with respect to this embedding of $G[C \cup\{a\}]$. A chord $e=u v$ is crossing if it is a chord in the face $F$ (that produces a crossing involving $a$ in the plane embedding of $H_{a}-b$ inherited from the plane embedding of $C$ ) and $a$ has neighbors in different components of $F-\{u, v\}$. Recall the strong Tutte-Hannani theorem: a graph is planar if and only if it has an embedding so that no two vertex-disjoint edges cross an odd number of times. Applying the Tutte-Hannani to the fixed embedding of $G[C \cup\{a\}]$, it follows that there must be two disjoint edges that cross exactly once.

Lemma 1 yields $d_{G}(w) \geqslant 3$. Let $w_{1}, w_{2}$ be the two neighbors of $w$ in $S$ chosen consecutively in the circular ordering of the neighbors of $w$ determined by the plane embedding of $C$ so that $a$ has been placed in the face, $F_{1}$, using the edges $w_{1} w$ and $w w_{2}$. By the crossing-minimizing placement of $a$, there must be a neighbor of $a$ in $F_{1}$, call it $a_{1}$ (possibly $\left.a_{1} \in\left\{w_{1}, w_{2}\right\}\right)$. Because there is a crossing, there must be a neighbor of $a$ outside of $F_{1}$, call it $a_{2}$. We may choose $a_{2}$ to be the first neighbor of $a$ clockwise from $w_{2}$ (away from $w_{1}$ ) along $F$ that is outside of $F_{1}$. Let $w_{3}$ be the next neighbor (clockwise) of $w$ that is at least as far as $a_{2}$. If $a$ has a neighbor in $F_{1}-\left\{w_{1}, w_{2}\right\}$, then delete all edges incident to $w$ except $w_{1} w$ and $w w_{2}$ and then contract these two edges to produce a crossing chord in this minor of $G$. Otherwise we may assume all neighbors of $a$ in $F_{1}$ are in $\left\{w_{1}, w_{2}\right\}$, in particular, we may assume $a_{1}=w_{1}$. If $a_{2} \neq w_{3}$, then contracting $w_{1} w$ and $w w_{3}$ produces a crossing chord in a minor of $G$. So assume $\left\{a_{1}, a_{2}\right\}=\left\{w_{1}, w_{3}\right\}$. If $N_{J}(w)=\left\{w_{1}, w_{2}, w_{3}\right\}$ and $N_{J}(a)=\left\{w_{1}, w_{3}\right\}$, then a crossing-free drawing of $G[C \cup\{a\}]$ could be made by placing $a$ in the face defined by the edges $w_{1} w$ and $w w_{3}$. If $w$ has another neighbor, $w_{4}$, then contracting $w_{2} w$ and $w w_{4}$ produces a crossing chord in a minor of $G$. Otherwise $N_{J}(w)=\left\{w_{1}, w_{2}, w_{3}\right\}$, and all of $a$ 's neighbors are in the face determined by $w_{1} w$ and $w w_{3}$.

So the only case in which we cannot create a minor with a crossing chord is when $a$ and $w$ are both branch of $H_{b}$, which is a subdivided $K_{3,3}$, and $N(a) \cap F=\left\{w_{1}, w_{2}, w_{3}\right\}=$ $N(w) \cap F$.

If $H_{a}$ is a subdivided $K_{5}$, then $F$ has three branch vertices of $H_{a}$ which partition $F$ into three paths. If $w_{i}, w_{j}$ with $1 \leqslant i<j \leqslant 3$ are on the same path of $H_{a}$, then rerouting this path through $w_{i}, w_{j}$ and $w$ produces another $H_{a}$ that has smaller $|\operatorname{int}(F)|$. So the only remaining case is if $w_{1}, w_{2}$, and $w_{3}$ are interior vertices on the three different paths of $H_{a}$ on $F$. This is shown on the left panel of Figure 27.

Adding $a$ (and its incident edges to the neighbors of $w$ ) produces graph (shown in right panel of Figure 27) which remains non-planar after deleting $y$, a contradiction.

Consider now the case in which $H_{a}$ is a subdivided $K_{3,3}$. If $w_{i}, w_{j}$ with $1 \leqslant i<j \leqslant 3$ are on the same path of $H_{a}$, then rerouting this path through $w_{i}, w_{j}$ and $w$ produces another $H_{a}$ with smaller $|\operatorname{int}(F)|$. So the only remaining case is if $w_{1}, w_{2}$, and $w_{3}$ are interior vertices on the three different paths of $H_{a}$ on $F$. The non-isomorphic cases are shown on the left panel of Figure 28. 

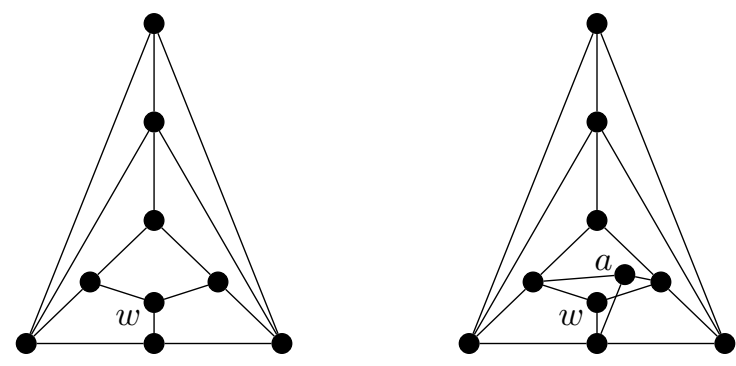

Figure 27: $H_{a}$ is a subdivided $K_{5}$ and the neighbors of $w$ are interior vertices on the three paths of $H_{a}$ on $F$ (left). Adding $a$, produces graph shown in center panel which remains non-planar after deleting $y$ (top vertex).
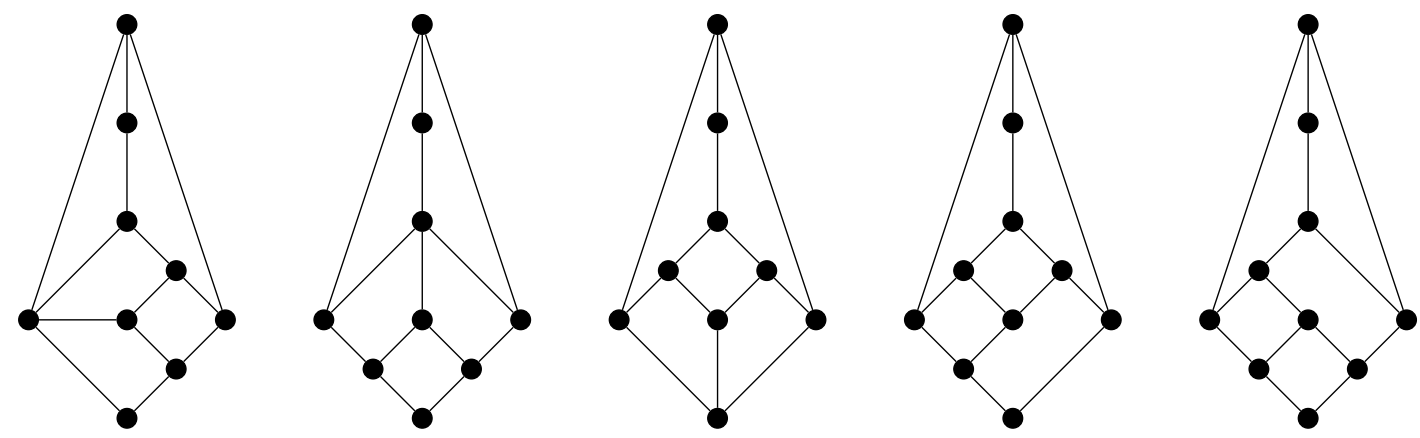

Figure 28: $H_{a}$ is a subdivided $K_{3,3}$ and the neighbors of $w$ are interior vertices on the three different paths of $H_{a}$ on $F$. The non-isomorphic cases are shown here.

In each case it is possible to find a new subdivision of $K_{3,3}$ for $H_{a}$ (in which $x$ and $y$ are branch vertices connected by a path through $b)$ with smaller $|\operatorname{int}(F)|$ as shown in Figure 29.

If $a$ has neighbors in $C$ that are all in a single path of $H_{a}-b$, then a technical difficulty arises: $\bar{a}$ indeed hits only one face (as Step 1 guarantees), but there are two choices for this one face - either of the two faces incident to this path (in the unique plane embedding of $\left.H_{a}-b\right)$. The next step excludes this possibility, resolving this difficulty.

A path in $C$ whose internal vertices are disjoint from $H_{a}-b$ is called an external path.

STEP 5: The neighbors of $a$ in $C$ are not all on one path of $H_{a}-b$.

Assume, to the contrary, that the neighbors of $a$ in $C$ are all on one path of $H_{a}-b$. Because $t=1$, every crossing pair of edges involves a crossing chord and an edge incident to $a$. Keep in mind that, by the minimality of $H_{a}$, the endpoints of any crossing chord cannot both be on the same path connecting branch vertices of $H_{a}$.

Let $F_{1}$ and $F_{2}$ be the two faces (in the unique plane embedding of $H_{a}-b$ ) incident to the path $P$ (connecting branch vertices $H_{a}-b$ ) that contains all of the neighbors of $a$ in $C$. It is very important to note that Steps $2-4$ apply to both $F_{1}$ and $F_{2}$. In particular, this means that no vertices of $C$ are in the interior of $F_{1}$ or $F_{2}$; so these faces of the plane embedding of $H_{a}-b$ are actually faces of the plane embedding of $C$. As in prior steps, 

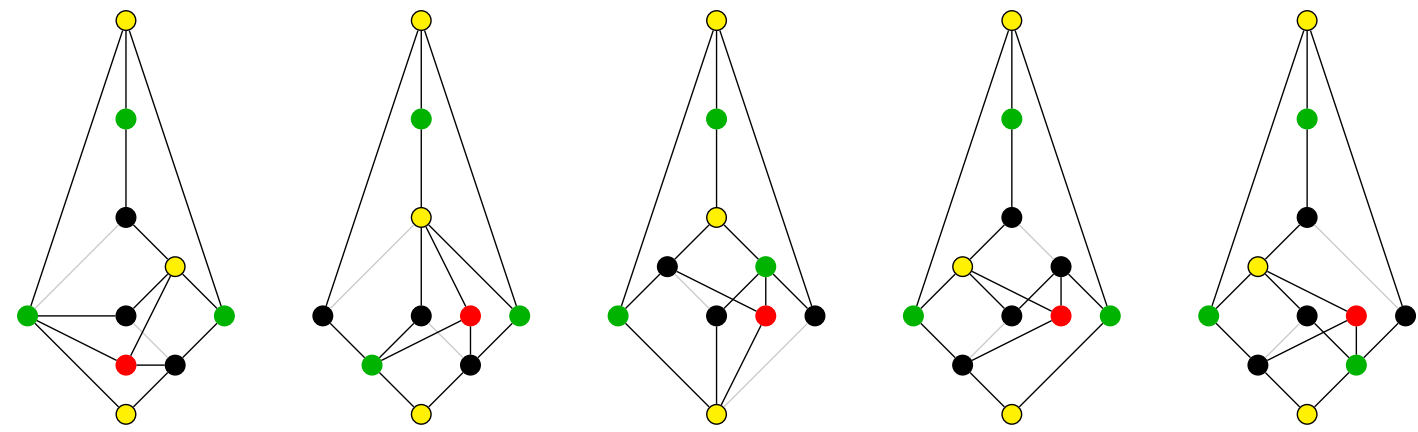

Figure 29: In each case a new subdivision of $K_{3,3}$ can be chosen for $H_{a}$ with smaller $|\operatorname{int}(F)|$. The branch vertices of the new subdivision of $K_{3,3}$ are shown in green and yellow. Vertex $a$ is shown in red. The non-branch vertex $b$ of this $K_{3,3}$ subdivision, which connects $x$ and $y$, is not shown.

we now consider cases depending on whether $H_{a}-b$ is a subdivision of $K_{5}-e$ or $K_{3,3}-e$.

First consider the easier case in which $H_{a}-b$ is a subdivision of $K_{5}-e$. Suppose that $P$ is a path ending at $x$. Let $a_{1}$ and $a_{2}$ be the extreme neighbors that $a$ has on $P$; that is, $a_{1}$ is the furthest from $x$ and $a_{2}$ is the closest to $x$. There must be a chord in $F_{1}$ that produces a crossing with $a$. This chord must have one endpoint between $a_{1}$ and $a_{2}$. The other endpoint cannot be on $P$ by the minimality of $H_{a}$. There must be a chord in $F_{2}$ with similar properties. These chords are drawn in color in Figure 30. Regardless of where the other endpoints of these chords occur, contractions produce the graph on the right of Figure 30, a $K_{5}$ minor that avoids $y$, a contradiction.
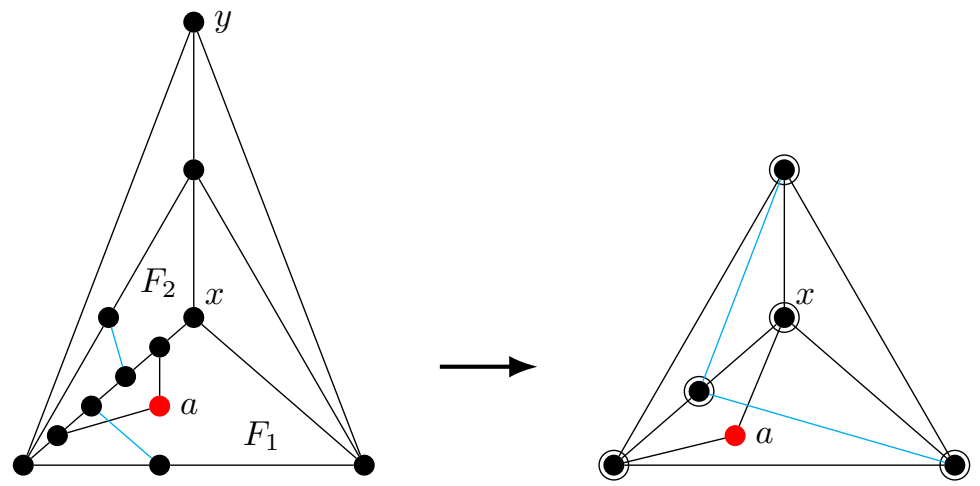

Figure 30: $H_{a}-b$ is a subdivision of $K_{5}-e$ and $a$ has neighbors in $C$ only along a path of $H_{a}-b$ ending at $x$. A $K_{5}$ minor avoiding $y$ appears.

So suppose that $P$ is a path of $H_{a}-b$ that does not end at $x$. Again $F_{1}$ and $F_{2}$ have chords that produce crossing with $a$ (see left of Figure 31). Regardless of where the other endpoints of these chords occur, contractions produce the graph shown in the center of Figure 31. Adding the vertex $b$ and the edge $a b$ (which corresponds to contracting the light component) produces the apex obstruction $M$ as a minor of $G$, a contradiction. 

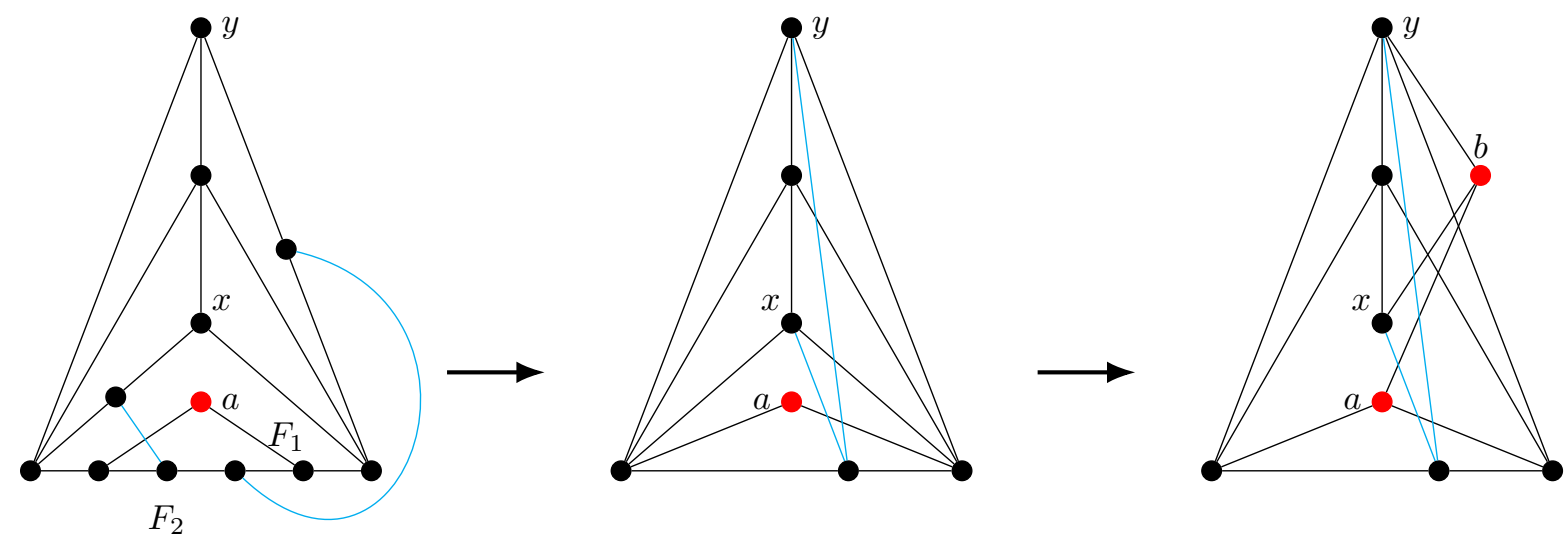

Figure 31: $H_{a}-b$ is a subdivision of $K_{5}-e$ and $a$ has neighbors in $C$ only along a path of $H_{a}-b$ that does not end at $x$. An $M$ minor appears after adding $b$ and the edge $a b$ (which corresponds to contracting the light component).

Next consider the case in which $H_{a}-b$ is a subdivision of $K_{3,3}-e$. Two subcases occur depending on whether the path $P$ (containing all neighbors of $a$ in $C$ ) is incident to $x$ or $y$, or neither $x$ nor $y$.

First consider the subcase in which $P$ is incident to neither $x$ nor $y$. Again define $a_{1}$ and $a_{2}$ to be the extreme neighbors of $a$ along $P$; that is, the neighbors of $a$ closest to the branch vertices of $H_{a}-b$ at the ends of $P$. As before, $F_{1}$ and $F_{2}$ must contain chords producing crossings with edges incident to $a$ (see Figure 32) These chords must have ends in the interval of $P$ between $a_{1}$ and $a_{2}$; call these ends $\alpha$ and $\beta$. The vertices at the other ends of these chords are of three possible types,

type (I): can be contracted to $x$ or $y$ along paths in $H_{a}-b$ (without passing through a branch vertex of $\left.H_{a}-b\right)$,

type (II): appear in the interior of the path in $H_{a}-b$ opposite $x$ or $y$ (along the face $F_{1}$ or $\left.F_{2}\right)$,

type (III): branch vertex of $H_{a}-b$ (adjacent to $x$ or $y$ in $H_{a}-b$ ).

First we argue that $\alpha=\beta$. If $\alpha \neq \beta$, then we claim that the edges along $P$ can be contracted, preserving $H_{a}$, so that a Kuratowski avoiding $b$ exists. This is a contradiction because then neither $a$ nor $b$ is an apex in $G[C \cup\{a, b\}]$ after contracting $\alpha$ to $\beta$. All nine cases (two end vertices each have independently any of three types) can be dismissed by examining graphs shown in Figure 33. All graphs in this figure have $a_{1}$ and $a_{2}$ contracted (if necessary) to the ends of the path $P$ and $\alpha$ contracted to $\beta$. If both ends of the crossing chords are type (I), the leftmost graph shows that there is a minor of $K_{3,3}$ avoiding $b$. If the ends of the crossing chords are both type (III), the right graph shows that there is a minor of $K_{5}$ avoiding $b$. The right-most graph also works if one chord is of type (I) and the other is of type (III). If one end of a crossing chords is type (I) and other is type (II), 


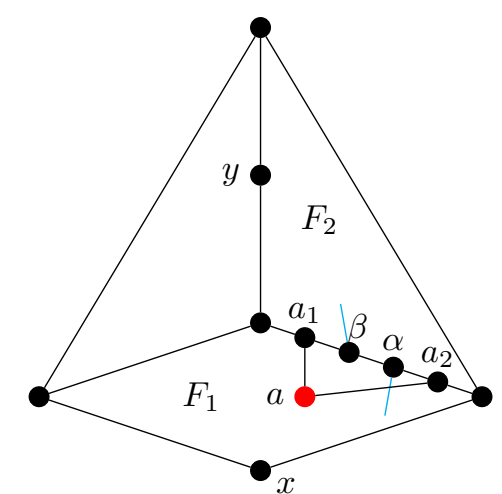

Figure 32: The case in which $H_{a}-b$ is a subdivision of $K_{3,3}-e$ and $a$ has neighbors in $C$ only along a path of $H_{a}-b$ that ends neither at $x$ nor $y$.
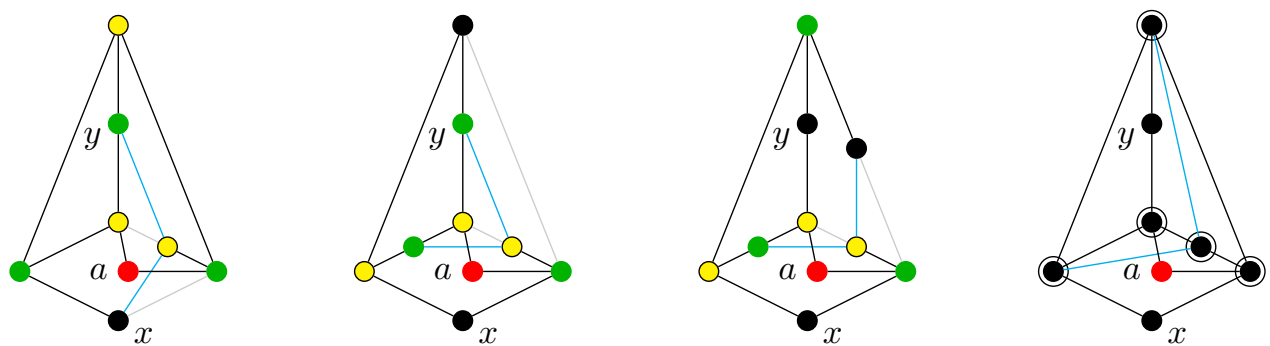

Figure 33: If $\alpha \neq \beta$, then edges along $P$ can be contracted, preserving $H_{a}$, so that a Kuratowski subgraph avoiding $b$ emerges.

the left-center graph shows that there is a minor of $K_{3,3}$ avoiding $b$. If one end is of type (II) and other is type (II) or (III), then the right-center graph of Figure 33 shows a minor of $K_{3,3}$ avoiding $b$.

So we may assume that $\alpha=\beta$.

If both chords have ends that are of type (I), then an edge $e$ exists (shown dotted in Figure 34) such that $J-e-a$ and $J-e-b$ are non-planar, contradicting Lemma 17 part (i). This can be seen in Figure 34 where a subdivision of $K_{3,3}$ is found in both $J-e-a\left(\right.$ new $\left.H_{a}\right)$, and $J-e-b\left(\right.$ new $\left.H_{b}\right)$.
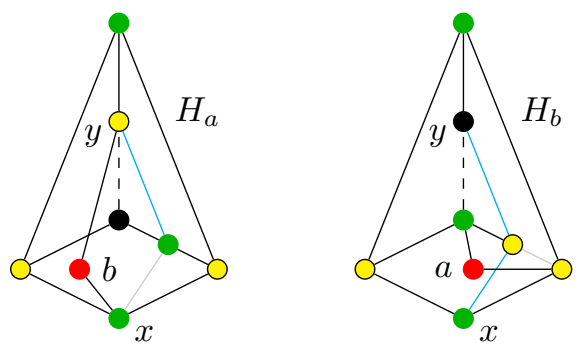

Figure 34: If $\alpha=\beta$ and both crossing chords have ends that are of type (I), then a choice of $H_{a}$ and $H_{b}$ are shown that avoid an edge of $C$ (shown dotted). 
If both chords have ends that are of type (III), then an edge $e$ exists (shown in pink in Figure 35) such that $J / e-a$ and $J / e-b$ are non-planar, contradicting Lemma 17 part (ii). This figure also shows a contradiction if one end of a crossing chord has type (III).
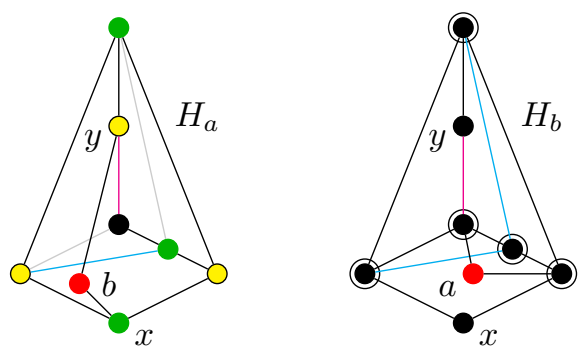

Figure 35: If $\alpha=\beta$ and one crossing chord has type (III), then a choice of $H_{a}$ and $H_{b}$ are shown that a share a contractible edge (shown in pink).

If the end of one crossing chord has type (I) and the other is of type (II), then an edge $e$ exists (shown dotted in Figure 36) such that $J-e-a$ and $J-e-b$ are non-planar, contradicting Lemma 17 part (i).
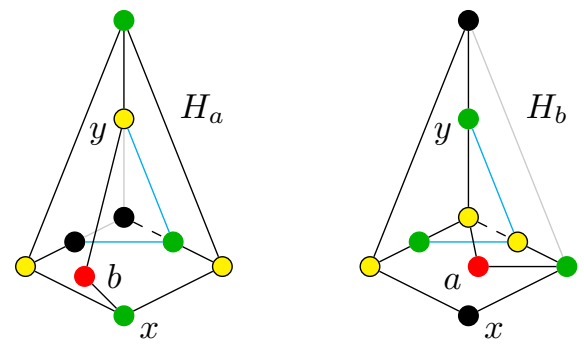

Figure 36: If $\alpha=\beta$ and one crossing chord has type (I) and the other has type (II), then a choice of $H_{a}$ and $H_{b}$ are shown that a share a deletable edge (shown dotted).

If both ends of the crossing chords have type (II), then an edge $e$ exists (shown in pink in Figure 37) such that $J / e-a$ and $J / e-b$ are non-planar, contradicting Lemma 17 part (i).
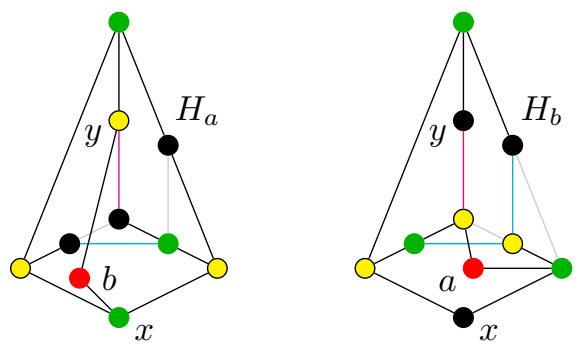

Figure 37: If $\alpha=\beta$ and both ends of the crossing chords have type (II), then a choice of $H_{a}$ and $H_{b}$ are shown that a share a contractible edge (shown in pink). 
Finally consider the subcase in which $a$ has neighbors only along the path $P$ of $H_{a}-b$ and $P$ is incident to $x$ or $y$. Without loss of generality, the path $P$ is incident to $x$. There are two possibilities, shown in Figure 38, according to whether $a$ has neighbors on one side of $x$ (along $P$ ) or both sides of $x$. Figure 38 introduces the labeling of the remaining branch vertices of $H_{a}$ as $p, q, r, s$ as well as the extreme neighbors, $a_{1}$ and $a_{2}$, of $a$ along $P$, as shown.
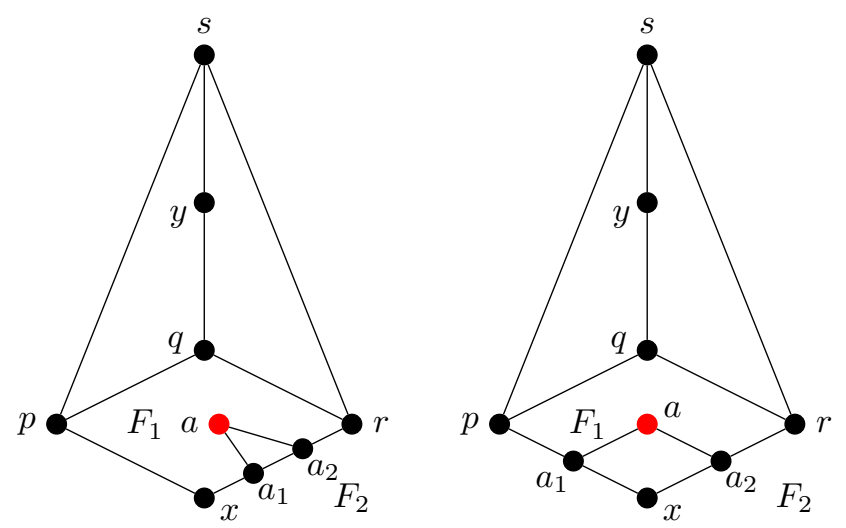

Figure 38: The two possibilities when $H_{a}-b$ is a subdivision of $K_{3,3}-e$, the path $P$ is incident to $x$, and $a$ has neighbors on one side of $x$ (left) or both sides of $x$ (right).

Recall that there must be a crossing chord in both faces $F_{1}$ and $F_{2}$ preventing $a$ from being placed into each face. By the minimality of $H_{a}$, these chords must have ends on distinct paths of $H_{a}$.

Regarding the leftmost graph in Figure 38, we may assume that $a_{1}=x$ and $a_{2}=r$, since contracting edges along the $\left[x, a_{1}\right]$ and $\left[a_{2}, r\right]$ segments of $P$ cannot destroy crossing chords. The crossing chords must have one endpoint between $a_{1}$ and $a_{2}$ along $P$ and another endpoint outside this interval. For the $F_{1}$ face, the other endpoint is contractible to $p$ or to $q$. For the $F_{2}$ face, the other endpoint is contractible to $p$ or to $s$. The four resulting cases are depicted in Figure 39.

In all cases, except the bottom right case of Figure 39, the ends of the crossing chords must be distinct because the chords must cross each other as well cross edges incident to $a$. If the chords did not cross each other, then they could be brought into a single face. In the bottom right case, we may contract the ends of the crossing chords in the $(x, r)$-interval of $P$ to the same vertex. As the figure shows, all four cases result in a $K_{3,3}$ subdivision avoiding $y$, a contradiction.

In the final case of Step 5, consider the rightmost graph in Figure 38. This graph can be redrawn as shown in Figure 40; the ends of the crossing chords are left undecided in this drawing. 


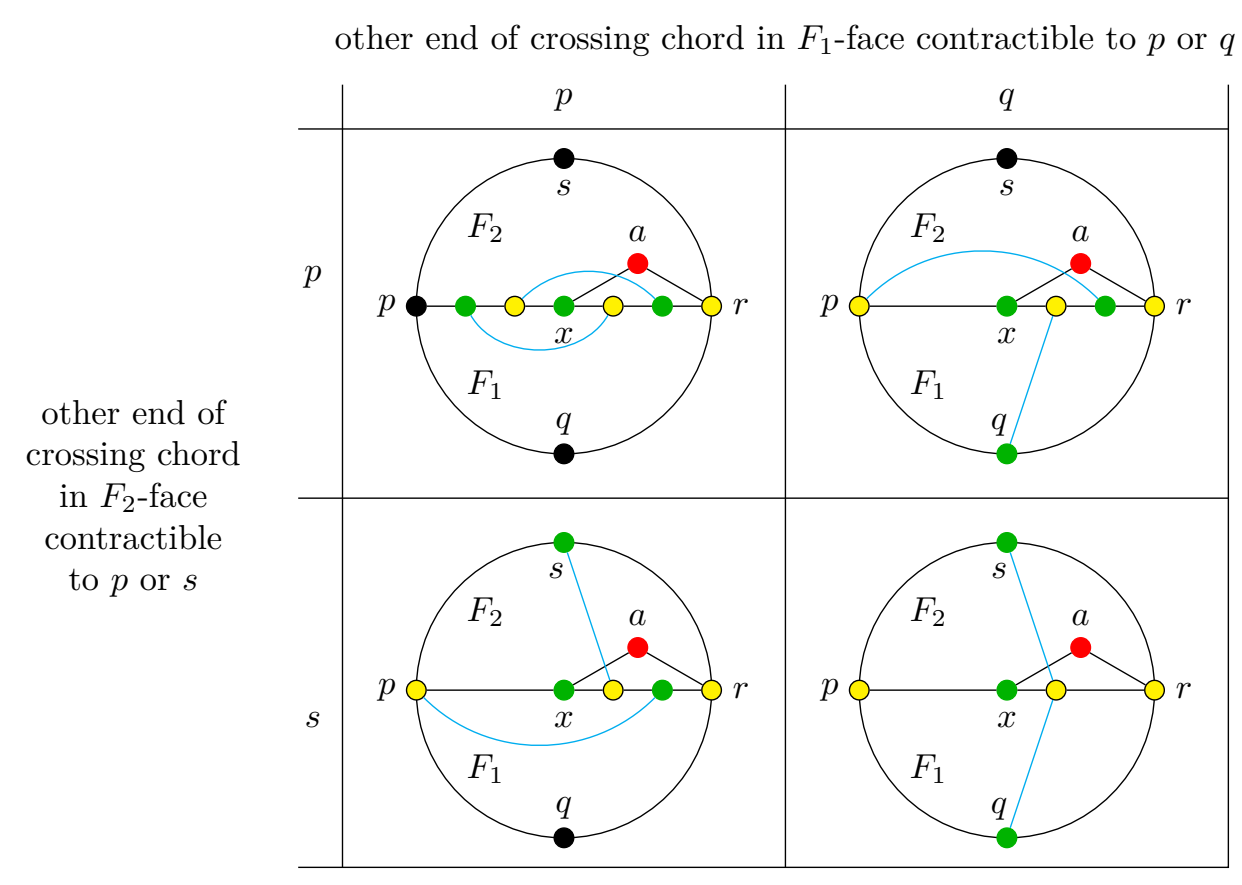

Figure 39: The four possible combinations for ends of crossing chords (shown in light blue).

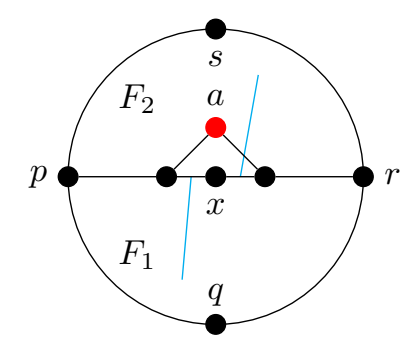

Figure 40: A redrawing of a portion of the rightmost graph from Figure 38 with crossing chords shown in light blue.

If the ends of the chords can be contracted to $s$ and $q$, then a minor isomorphic to $M$ (a Petersen-family graph) in $G$ emerges (Figure 41) after adding $b$ and the edge $a b$, the latter of which corresponds to contracting the light component of $G$.

In the remaining possible placements of the ends of the crossing chords, each possibility can be contracted to one of the two shown in Figure 42. Observe that the crossing chords must again cross each other (as well as edges incident to $a$ ) to avoid being placed into a single face. In both cases, a $K_{3,3}$ avoiding $y$ is shown, a contradiction. 


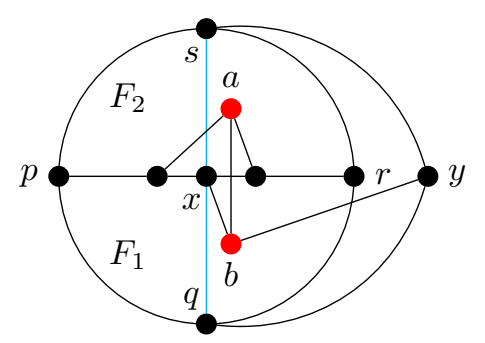

Figure 41: If the crossing chords have ends that can be contracted to $s$ and $q$, then a minor of $M$ emerges.
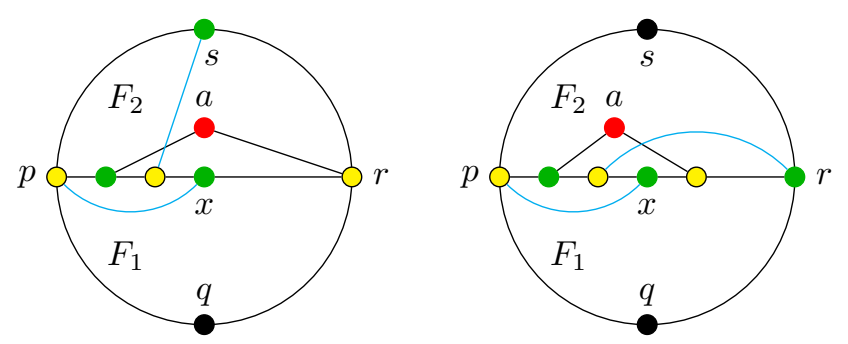

Figure 42: Crossing chords must cross each other in the final two possible placements of the ends of these crossing chords (shown in blue). The cases can each be contracted to the graphs depicted, or similar graphs. A $K_{3,3}$ avoiding $y$ arises in both cases.

\section{STEP 6: The final contradiction to complete the proof.}

In this final step, we may assume that $a$ has neighbors in only one face of the plane embedding of $H_{a}-b$ (Step 1). Furthermore there are no other vertices of $C$ in this face (Steps 2-4). Finally we may assume that all of $a$ 's neighbors in $C$ are not along a single path of $H_{a}-b$ (Step 5). Consequently, there is exactly one face, $F$, containing all of the neighbors of $a$ in $C$ and there must be a crossing chord in $F$.

If $H_{a}$ is a subdivision of $K_{5}$, then there are four remaining non-isomorphic positions for a crossing chord. These cases are shown in Figure 43.
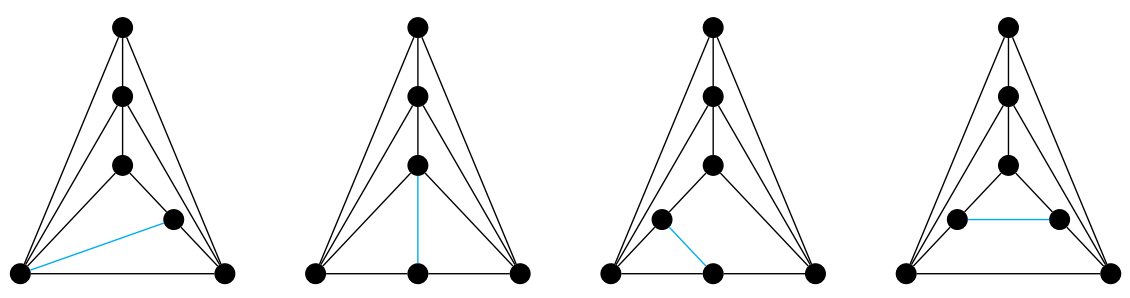

Figure 43: $H_{a}$ is a subdivision of $K_{5}$. The four non-isomorphic positions for the crossing chord (shown in light blue) when endpoints are not along the same path of $H_{a}$.

In each of these cases (see Figure 43), a new $K_{3,3}$ subdivision for $H_{a}$ exists with $x$ and $y$ branch vertices connected by $b$ (see Figure 44 ). One can argue in each case that this new 
$H_{a}$ has a unique embedding of $H_{a}-b$ such that $a$ now hits multiple faces, contradicting Step 1. This argument requires some care. For example, in the left-most graph, clearly $a$ must have neighbors that cross the blue chord. These could simply be a vertex along the $(u, x)$-path and another along the $(u, v)$-path. However all of the neighbors of $a$ could not be along these paths by Step 5 .
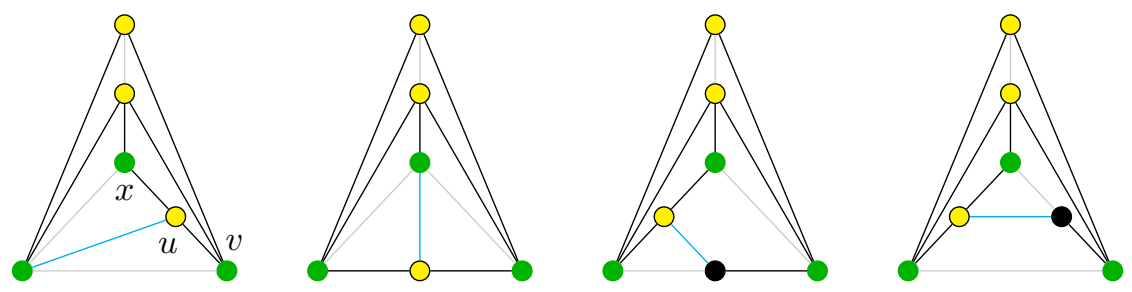

Figure 44: In each case of Figure 43 a $K_{3,3}$ subdivision for $H_{a}$ exists with $x$ and $y$ branch vertices connected by $b$.

Now consider the ten non-isomorphic ways a crossing chord can appear in $F$ when $H_{a}$ is a subdivision of $K_{3,3}$. Six of these cases (see Figure 45) can be dismissed using an argument similar to the one given in the prior paragraphs.
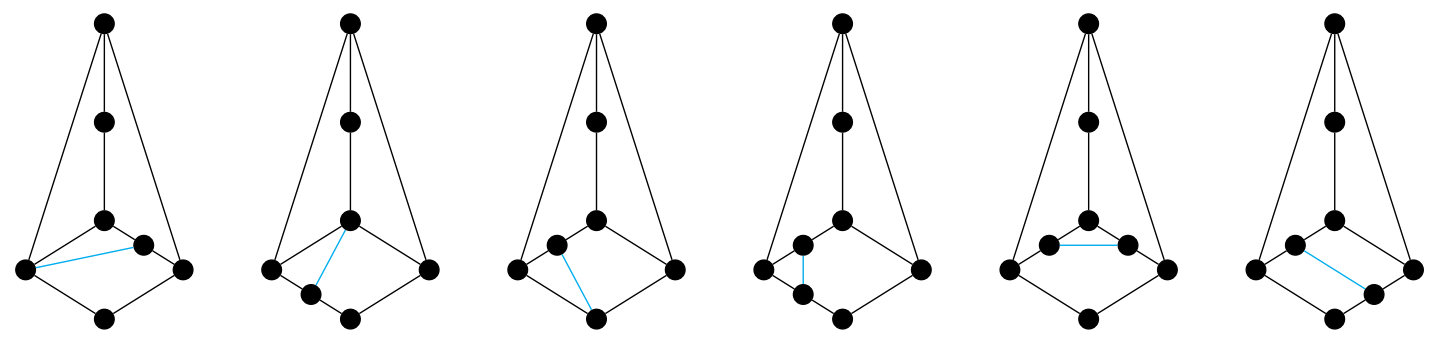

Figure 45: Six of the ten non-isomorphic ways a crossing chord (shown in light blue) can appear in $F$ when $H_{a}$ is a subdivision of $K_{3,3}$.

Each case of Figure 45 has a new $K_{3,3}$ subdivision with $x$ and $y$ branch vertices connected by $b$. This new $H_{a}-b$ has $a$ that now hits multiple faces (by Step 5), contradicting Step 1 (see Figure 46);
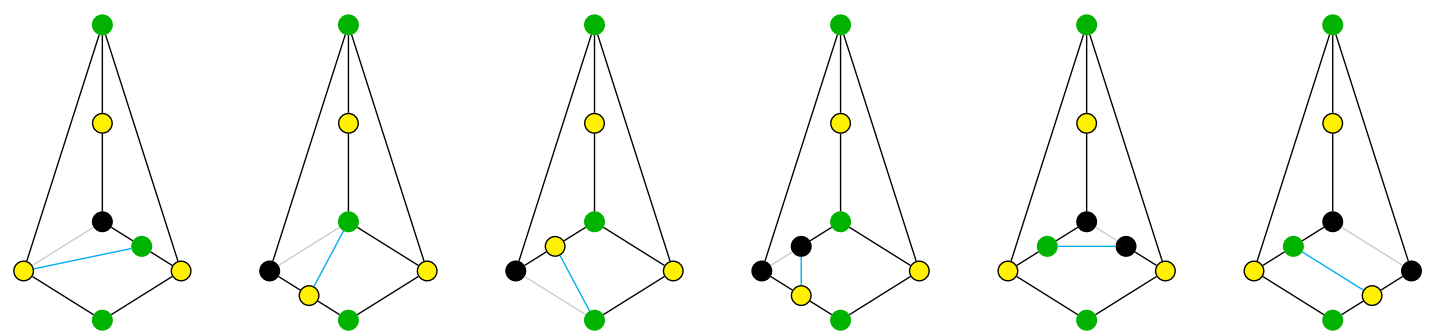

Figure 46: Each case of Figure 45 has a new $K_{3,3}$ subdivision with $x$ and $y$ branch vertices connected by $b$. This new $H_{a}-b$ has $a$ that now hits multiple faces, contradicting Step 1 . 
The last four remaining cases are shown in Figure 47.
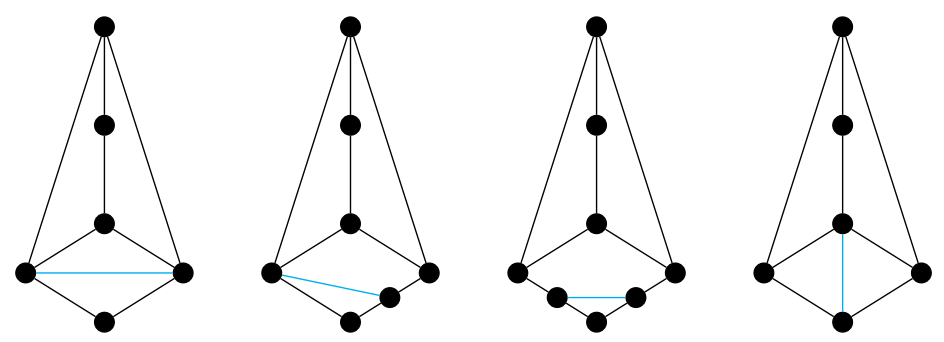

Figure 47: The four remaining chord placements in $H_{a}-b$.

Consider left-most graph in Figure 47 in which $u v$ is the crossing chord. This means that $a$ has a neighbor on the upper half (above the chord $u v$ ) of face $F$ and a neighbor on the lower half (below the chord $u v$ ) of face $F$. Observe that there must be an external path $P$ that connects the lower open interval $(v, u)$ of the exterior face (shown in red in Figure 48) to the upper open interval $(u, v)$ of the exterior face (shown in green in Figure 48); otherwise the $u v$ chord could be drawn on the exterior face, reducing the number of crossings produced when reinserting $a$ into the planar embedding of $C$; that is, the external path $P$ blocks the chord $u v$. The resulting graph can be contracted to the one shown on the right of Figure 48. Adding vertex $a$ to this graph produces a $K_{5}$ minor of $G$ which implies that $H_{a}-b=H_{b}-a$ in the original graph $G$, so $\{x, y\}$ is another 2-cut of $G$, a contradiction.
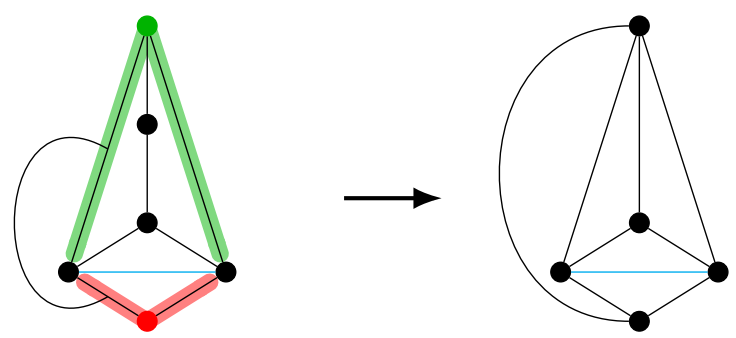

Figure 48: An external path connecting the lower exterior face (red) to the upper exterior face (green) implies a $K_{5}$ minor of $G$.

Consider next the center-left graph in Figure 47. Again let $u v$ be the crossing chord; let $w$ be the branch vertex of $H_{a}-b$ opposite $u$ on face $F$ (as shown in Figure 49).

The vertex $a$ has neighbors on $F$ above and below the chord $u v$ because $u v$ is a crossing chord. By Step 5, $a$ has neighbors between $u$ and $w$ (on the upper part of $F$ - Figure 49 shows one case); otherwise all of $a$ 's neighbors would occur on the path of $H_{a}-b$ containing $x$. Now the same analysis as given in the prior paragraph (Figure 48) applies. We omit further details for this case. Similar reasoning applies to the center-right case shown in Figure 47.

The final analysis regards the case in which the crossing chord is a vertical through $F$ connecting branch vertices of $H_{a}-b$ (shown as the rightmost graph of Figure 47). By 


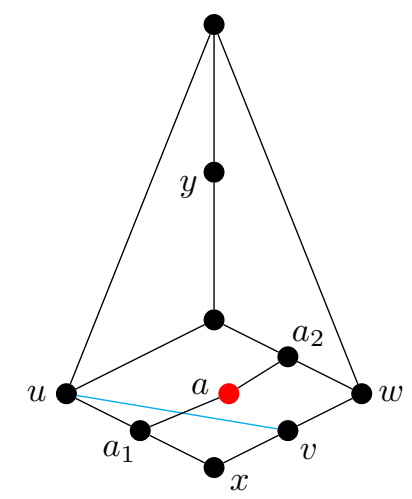

Figure 49: By Step 5, the vertex a must have neighbors above uw and below the blue crossing chord.

Step 5 not all of $a$ neighbors can occur on the path of $H_{a}-b$ containing $x$. Thus there are three cases that remain; these are shown in Figure 50.
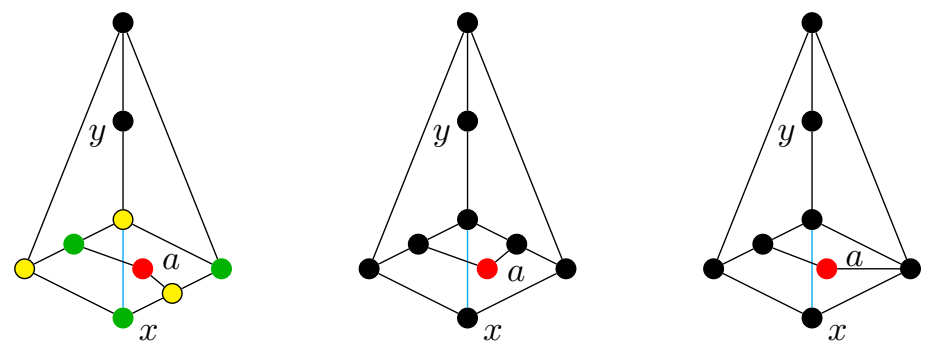

Figure 50: The left graph contains a subdivision of $K_{3,3}$ avoiding $b$ and $y$. The other two contract to $P_{7}$ after adding $b$ and the edge $a b$.

Observe that the left most graph of Figure 50 contains a subdivision of $K_{3,3}$ avoiding $b$ and $y$. The other two graphs contain a minor of $P_{7}$ (see Figure 22) after adding $b$ and the edge $a b$.

The next corollary strengthens the statement of Proposition 21 to show that vertices in the unique 2-cut must be branch vertices of all of their Kuratowski witnesses.

Corollary 22. If Assumptions 15 are satisfied, then $b$ is a branch vertex for any Kuratowski subgraph of $G$ avoiding a (and vice versa, a is a branch vertex for any Kuratowski subgraph of $G$ avoiding $b)$.

Proof. Note that $b$ must have at least three neighbors in $C$ since Proposition 21 guarantees a Kuratowski subgraph avoiding $a$ (with vertices all in $C \cup\{a\}$ ) in which $b$ must appear as a branch vertex. Suppose now that $H_{a}$ is an arbitrary Kuratowski subgraph of $G$ avoiding a. Clearly $V\left(H_{a}\right) \subseteq C \cup\{b\}$. If one of the edges in $G[C \cup\{b\}]$ incident to $b$ does not appear in $H_{a}$, then it is not in $E\left(H_{a}\right) \cup E\left(H_{b}\right)$, contradicting Lemma 16 part (iii). 


\subsection{Two Kuratowski subgraphs have branch vertices that cover $C \cup\{a, b\}$}

With Corollary 22, establishing that $a$ and $b$ must be branch vertices of all of their $\mathrm{Ku}-$ ratowski witnesses, we are almost ready to prove the main objective of this subsection: the existence of two Kuratowski subgraphs whose branch vertices cover $C \cup\{a, b\}$ (Theorem 25). First we focus on properties of vertices in $C$ that are not branch vertices of Kuratowski witnesses.

Lemma 23 (Non-Branch Vertex Lemma). Assume Assumptions 15 and $H_{a}$ and $H_{b}$ are Kuratowski subgraphs of $G$ avoiding $a$ and $b$, respectively. If $w \in C$ is a branch vertex of neither $H_{a}$ nor $H_{b}$ :

(i) $d_{G}(w)=4$, with $w$ incident to two edges in $E\left(H_{a}\right)$ and two in $E\left(H_{b}\right)$.

(ii) If $e=w x \in E\left(H_{a}\right)$ then $x \in V\left(H_{b}\right) \cup\{b\}, H_{b} \not \subset G / e$, and $b$ is the only apex for $G / e$.

(iii) If $e=w x \in E\left(H_{b}\right)$ then $x \in V\left(H_{a}\right) \cup\{a\}, H_{a} \not \subset G / e$, and $a$ is the only apex for $G / e$.

Proof. (i) Assume that $c \in C$ is not a branch vertex of $H_{a}$ or $H_{a}$. So the degree of $c$ in $H_{a}$ and $H_{b}$ is two. Lemma 1 implies $d_{G}(w) \geqslant 3$. If $d_{G}(w)>4$, then there is an edge of $G[C \cup\{a, b\}]$ that is not covered by $H_{a}$ or $H_{b}$ contradicting Lemma 16 part (iii). To prove claim (i), it suffices to prove that $d_{G}(w) \neq 3$. Assume, to the contrary, that $d_{G}(w)=3$. The pigeon-hole principle guarantees an edge $w x \in E\left(H_{a}\right) \cap E\left(H_{b}\right)$ and so $x \notin\{a, b\}$. Note that $G / w x$ must have an apex $z$ in $H_{a} / w x \cap H_{b} / w x$, so $z \notin\{a, b\}$. However, $z$ must also separate $a$ from $b$ in $G[C \cup\{a, b\}]$ since otherwise $L^{+}$would still be a minor of $G / w x$. But then either $\{a, z\}$ or $\{b, z\}$ is another 2-cut of $G$ (contradicting that $S$ is the only 2-cut) or $z$ is the vertex resulting from the contraction of the edge $w x$, contradicting Lemma 16 part (iv).

(ii-iii) By symmetry, it suffices to prove (ii). Consider $e=w x \in E\left(H_{a}\right)$. If $x \notin$ $V\left(H_{b}\right) \cup\{b\}$, then $H_{a}$ and $H_{b}$ remain Kuratowski subgraphs in $G / e$ implying that any apex $z$ for $G / e$ must be in $H_{a} \cap H_{b} \subset C$. However, this means that $z$ must also separate $a$ from $b$ in $G[C \cup\{a, b\}]$ since otherwise $L^{+}$would still exist in $G / e$. But then either $\{a, z\}$ or $\{b, z\}$ is another 2-cut of $G$ (contradicting that $S$ is the only 2-cut) or $z$ is the vertex resulting from the contraction of the edge $w x$, contradicting Lemma 16 part (iv). Therefore $x \in V\left(H_{b}\right) \cup\{b\}$. The reader can check that similar reasoning applies if $H_{b}$ remains a Kuratowski subgraph of $G / e$ or if $b$ is not the only apex for $G / e$.

Theorem 24. Assume Assumptions 15. Choose any Kuratowski subgraphs $H_{a}$ and $H_{b}$ avoiding $a$ and $b$, respectively, that also minimize $\left|E\left(H_{a}\right)\right|+\left|E\left(H_{b}\right)\right|$. If $H_{a}$ is a subdivision of $K_{5}$ or $H_{b}$ is a subdivision of $K_{5}$, then any vertex in $C$ is a branch vertex of $H_{a}$ or a branch vertex of $H_{b}$.

Proof. Without loss of generality, $H_{a}$ is a subdivision of $K_{5}$. Assume, to the contrary, there is a vertex $w \in C$ that is a not a branch vertex either $H_{a}$ or $H_{b}$. By Theorem 23 
part (i), $\operatorname{deg}_{G}(w)=4$ and $w \in V\left(H_{a}\right) \cap V\left(H_{b}\right)$. Now $w$ has two neighbors in $H_{b}$, at least one of which is not vertex $a$. Consider a neighbor $x$ of $w$ such that $w x \in E\left(H_{b}\right)$ and $x \neq a$. By Theorem 23 part (iii), $x \in V\left(H_{a}\right)$. There are three cases shown in Figure 51.
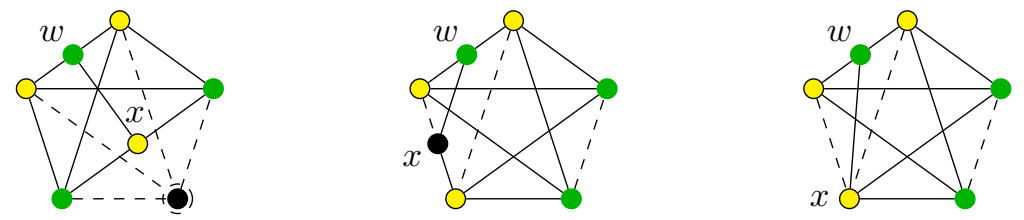

Figure 51: Deleting dotted lines/vertex and adding the edge $w x$ reveals a subdivision of $K_{3,3}$ with fewer edges than the original subdivision of $K_{5}$.

The figure shows a subdivision of $K_{5}$ that represents $H_{a} ; w$ is a non-branch vertex with a neighbor $x$ such that $w x \in E\left(H_{b}\right)-E\left(H_{a}\right)$. The vertex $x$ could be in any of three positions. The dotted lines indicates paths of $H_{a}$ that can be deleted leaving a new subdivision of $K_{3,3}$ with fewer edges than $H_{a}$, in each case. This new Kuratowski subgraph avoids vertex $a$ also, so contradicts the choice of $H_{a}$. So these cases essentially follow from a commonly rediscovered fact that a vertex minimal non-planar graph that is not just a subdivision of $K_{5}$ has a spanning $K_{3,3}$ subdivision.

In each case, a new choice of $H_{a}$ as a subdivision of $K_{3,3}$ has fewer edges than the current $H_{a}$; this contradicts that the original choice of $H_{a}$ and $H_{b}$ minimized $\left|E\left(H_{a}\right)\right|+$ $\left|E\left(H_{b}\right)\right|$.

The next theorem is the main result of this subsection.

Theorem 25. Assume Assumptions 15. There are Kuratowski subgraphs $H_{a}$ and $H_{b}$ avoiding $a$ and $b$ respectively, such that any vertex in $C \cup\{a, b\}$ is a branch vertex of $H_{a}$ or a branch vertex of $H_{b}$.

Proof. Choose Kuratowski subgraphs $H_{a}$ and $H_{b}$ as follows:

(i) $a \notin V\left(H_{a}\right)$ and $b \notin V\left(H_{b}\right)$,

(ii) maintaining (i), minimize $\left|E\left(H_{a}\right)\right|+\left|E\left(H_{b}\right)\right|$,

(iii) maintaining (i) and (ii), minimize $|W|$, where

$$
W=(C \cup\{a, b\})-\left\{v: v \text { is branch vertex of } H_{a} \text { or } H_{b}\right\} .
$$

It suffices to prove that this choice produces $W=\varnothing$. Corollary 22 implies $a, b \notin W$.

If $H_{a}$ is a subdivision of $K_{5}$ or $H_{b}$ is a subdivision of $K_{5}$, then Theorem 24 yields $W=\varnothing$. So, we may assume $H_{a}$ and $H_{b}$ are subdivisions of $K_{3,3}$.

Assume, to the contrary, that $W \neq \varnothing$. Let $w$ be an arbitrary vertex in $W$. If possible, choose $H_{a}$ and $H_{b}$, subdivisions of $K_{3,3}$, satisfying (i) - (iii), minimum $|W|$ and $w \in W$ so that $w \notin N(a)$. By Theorem 23, $d_{G}(w)=4, w \in V\left(H_{a}\right) \cap V\left(H_{b}\right)$, and all of the neighbors 


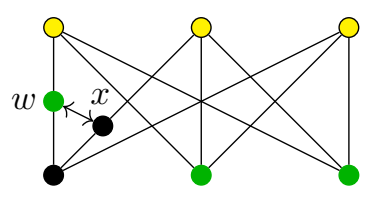

Figure 52: A depiction of $H_{a}$ and the extra edge $w x \in E\left(H_{b}\right)$. Contracting $w x$ preserves $H_{b}$ and non-planarity of $H_{a}$.

of $w$ are also vertices in $\left(V\left(H_{a}\right) \cap V\left(H_{b}\right)\right) \cup\{a, b\}$. Let $x, y$ be the neighbors of $w$ such that $w x, w y \in E\left(H_{b}\right)$. We may assume that $x \notin\{a, b\}$ because $w$ has two neighbors in $H_{b}$ and $b \notin V\left(H_{b}\right)$. Note also the minimality of $H_{a}$ implies that $x, y$ are not on the same path of $H_{a}$ that contains $w$.

Now consider $H_{a}$. If $w$ and $x$ are internal vertices of paths of $H_{a}$ that intersect at a common branch vertex, then contracting $w x$ preserves $H_{b}$ and also the non-planarity of $H_{a}$ (see Figure 52). This contradicts Lemma 19 part (ii).

Therefore $x$ must be a branch vertex of $H_{a}$ or it is an internal vertex on a path of $H_{a}$ that does not intersect at a common branch vertex with $w$.
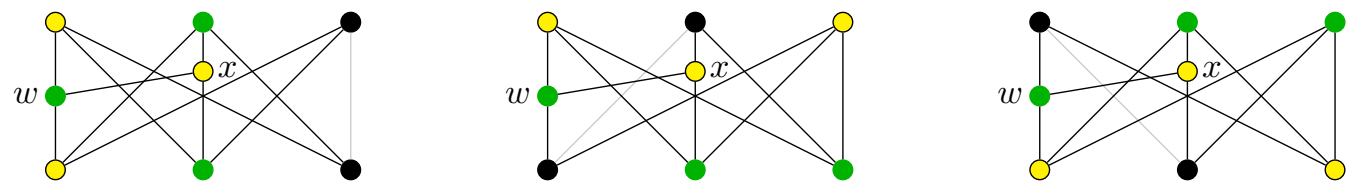

Figure 53: For any branch vertex $t$ of $H_{a}$, there is a subdivision of $K_{3,3}$ in $H_{a}+w x$ in which $t$ is not a branch vertex.

Assume that $x$ is not a branch vertex of $H_{a}$. In this case, Figure 53 shows that for any branch vertex $t$ of $H_{a}$, there is a subdivision of $K_{3,3}$ in $H_{a}+w x$ in which $t$ is not a branch vertex. Recall that $b$ is a branch vertex of $H_{a}$. So, if $x$ is not a branch vertex of $H_{a}$, then there exists a subdivision of $K_{3,3}$ missing $a$ that does not have $b$ as a branch vertex, contradicting Corollary 22.

Consequently we may assume that $x$ is a branch vertex of $H_{a}$ different from the ones at the end of the path of $H_{a}$ containing $w$. Without loss of generality $H_{a}$ appears as in see Figure 54, where the label $v$ has been introduced on the branch vertex of $H_{a}$ along the path of $H_{a}$ containing $w$ that has opposite color as $x$.

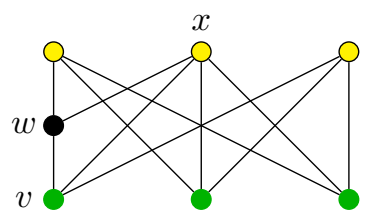

Figure 54: $x$ is a branch vertex of $H_{a}$ (different from the ones at the end of the path of $H_{a}$ containing $\left.w\right)$. 
Now consider the subdivision of $K_{3,3}$, call it $J_{v}$, in $H_{a}+w x$ that remains after deleting the edges along the $v x$-path (see Figure 55).

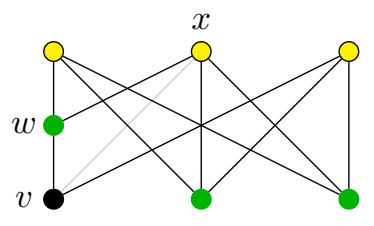

Figure 55: The graph $J_{v}$ that is a subdivision of $K_{3,3}$ in $H_{a}+w x$.

Note that $J_{v}$ covers all of the branch vertices of $H_{a}$ and does not use any vertices outside of $H_{a}$. By choice of $H_{a}$, there cannot be fewer edges in $J_{v}$ than in $H_{a}$. Consequently $J_{v}$ and $H_{a}$ must have the same number of edges. In particular the $v x$-path in $H_{a}$ is just the edge joining $v$ and $x$. Because $H_{a}$ minimizes $|W|$ and the branch vertices of $J_{v}$ and $H_{a}$ only differ at $v$ and $w$, the vertex $v$ is a branch vertex of neither $H_{b}$ nor $J_{v}$. In particular the edge $v x$ must be an edge of $H_{a}$ and $H_{b}$.

If $w$ and $v$ are both neighbors of $a$, a 4-cycle formed by $a, w, x$, and $v$ appears in $H_{b}$ (see Figure 56). However, since $w$ and $v$ are not branch vertices of $H_{b}$, this 4-cycle is an impossible configuration in $H_{b}$.

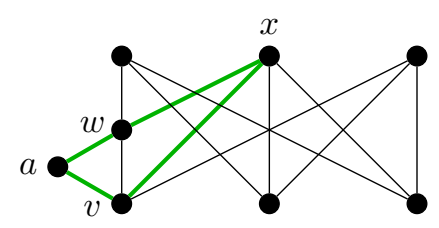

Figure 56: If both $w$ and $v$ are neighbors of $a$, then an impossible 4-cycle (green) appears in $H_{b}$.

By the choice of $w$ we conclude that $w \notin N_{G}(a)$ (if $w \in N_{G}(a)$ then replace $w$ with $v$ ). It follows from earlier reasoning that, like $x$, the other neighbor of $w$ in $H_{b}$, namely $y$, must also be a branch vertex of $H_{a}$.

If $x$ and $y$ were branch vertices of $H_{a}$ with the same color, then there would be a subdivision of $K_{3,3}$ with fewer edges than $H_{a}$ that could have been chosen (as shown in Figure 57) contradicting the choice of $H_{a}$.

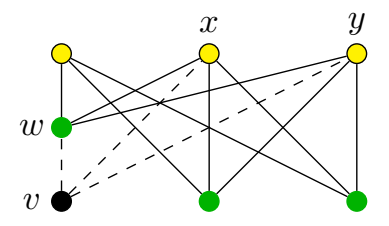

Figure 57: Deleting edges along the three dotted paths and adding the two edges $w x, w y$ produces a subdivision of $K_{3,3}$ with fewer edges than $H_{a}$. 
So, without loss of generality, $H_{a}$ appears as shown in Figure 58. This figure introduces labels for all of the branch vertices of $H_{a}$. Observe that, like $v$, the vertex $u$ is not a branch vertex of $H_{b}$. Also, like the edge $v x$, the edge $u y$ must be an edge in $H_{a}$ and $H_{b}$.

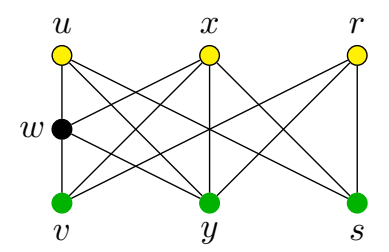

Figure 58: In $H_{a}$, the vertex $w$ must have two neighbors, $x$ and $y$, that are branch vertices of opposite color in $H_{a}$ and not on the path of $H_{a}$ containing $w$.

If $u, v \in N_{G}(a)$, then the edges $a u, u y, y w, w x, x v, v a$ form a 6 -cycle in $H_{b}$, as shown in Figure 59. Recall $a$ is a branch vertex of $H_{b}$ but $u, v$, and $w$ are not branch vertices of $H_{b}$. If $x$ or $y$ are not branch vertices of $H_{b}$, then a cycle with at most two branch vertices of $H_{b}$ exists in $H_{b}$, an impossibility. So, $a, x$ and $y$ are three branch vertices of $H_{b}$. However the 6-cycle induced by the edges $a u, u y, y w, w x, x v, v a$ from $H_{b}$ implies that $a$, $x$ and $y$ cannot be 2-colored as the branch vertices of a subdivision of $K_{3,3}$, contradicting that $H_{b} \cong T K_{3,3}$.

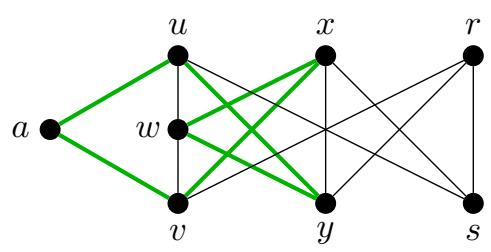

Figure 59: If $u, v \in N_{G}(a)$, then an impossible 6-cycle (shown in green) emerges in $H_{b}$.

Consequently $u \notin N_{G}(a)$ or $v \notin N_{G}(a)$. Without loss of generality, $v \notin N_{G}(a)$. Now $d_{G}(v)=4$ so $v$ has another neighbor in $V\left(H_{a}\right) \cap V\left(H_{b}\right)$. Applying the same reasoning to $v$ as we have applied previously to $w$, we conclude that the remaining unknown neighbor of $v$ must be a branch vertex of $H_{a}$. Further applying this reasoning to $J_{v}$ (see Figure 55) reveals that this neighbor of $v$ must be either $y$ or $s$. However, if $y$ is a neighbor of $v$ in $H_{b}$, then the four edges $v y, y w, w x, x v$ form an impossible 4-cycle in $H_{b}$ with at most two branch vertices (since $w$ and $v$ cannot be branch vertices of $H_{b}$ ). Therefore, $s$ must be the final neighbor of $v$ in $H_{b}$ and $v s$ must be an edge of $H_{b}$ (see leftmost graph in Figure 60).

Because $s \in N_{G}(v)$ there is a subdivision of $K_{3,3}$, call it $J_{r}$, that covers the branch vertices of $H_{a}$, contains only vertices from $H_{a}$, but does not have $r$ as a branch vertex (see middle of Figure 60). Applying the same reasoning to $J_{r}$ as we applied before to $J_{v}$, we conclude that $r$ is a branch vertex of neither $J_{r}$ nor $H_{b}$. Moreover the edge $r s$ is in $H_{b}$. Consequently the path uywxvsr has all its edges in $H_{b}$ and covers all of the branch vertices of $H_{a}$. In particular the vertices of this path must be in $H_{b}$. This is 

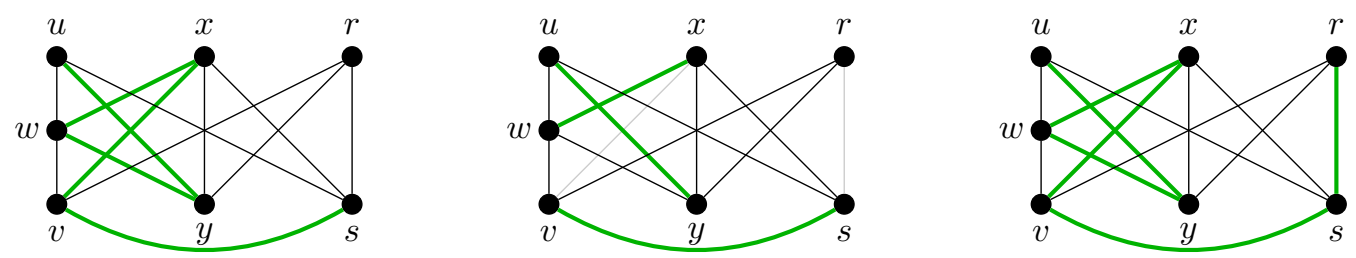

Figure 60: $s \in N_{G}(v)$ (left) implies a subdivision of $K_{3,3}$ (middle) that implies a path in $H_{b}$ that covers the branch vertices of $H_{a}$ (path shown in green edges at right).

a contradiction because $b$ is in $V\left(H_{a}\right)-V\left(H_{b}\right)$ and, by Corollary 22, it must also be a branch vertex of $H_{a}$. This contradiction proves that $W=\varnothing$, as desired.

\subsection{The final computation}

In this subsection we outline how to show the list of 72 connectivity- 2 apex obstructions satisfying Assumptions 15 is complete. While much of the case-analysis can be reduced by hand, ultimately we confirmed the final list using computers. We omit many details. Much of the case analysis applies to small (order $\leqslant 10$ ) graphs and is routine, but it is sufficiently tedious that it precludes comprehensive presentation.

Theorem 26. Suppose that $G \in \mathcal{F}, \kappa(G)=2, S=\{a, b\}$ is the unique 2-cut of $G, C$ is the heavy component of $G-S$ and $C$ is non-planar.

- If $G[C \cup\{a, b\}]$ has a 2-cut separating a from b, then $G$ is isomorphic to a graph in Figure 61.

- If $G[C \cup\{a, b\}]$ has no 2-cut separating a from b, then $G$ is isomorphic to a graph in Figure 62.

Proof. By Theorem 25, there are Kuratowski witnesses $H_{a}$ and $H_{b}$ in $G$ avoiding $a$ and $b$ respectively, such that any vertex in $C \cup\{a, b\}$ is a branch vertex of $H_{a}$ or a branch vertex of $H_{b}$. Consequently, every vertex of $G$ is a branch vertex of $H_{a}, H_{b}$, or the Kuratowski witness in $L^{+}$.

Because $a$ and $b$ are branch vertices of $H_{b}$ and $H_{a}$ respectively, it follows that $|C| \leqslant 10$. Indeed, the only way that $|C|=10$ is if $H_{a}$ and $H_{b}$ are subdivisions of $K_{3,3}$ with disjoint branch sets. This case can be shown never to occur by examining possible subdivided edges of $H_{a}$ and $H_{b}$ that must involve branch vertices from the other Kuratowski witness. We omit the details.

Clearly $|C| \geqslant 4$ because $H_{a}$, for one, has at least 5 branch vertices. If $|C|=4$, then it is easy to show $K_{6}$ is a minor of $G$, an impossibility. Thus it suffices to consider cases in which $5 \leqslant|C| \leqslant 9, C$ is connected and planar. There are 87,816 non-isomorphic, connected planar graphs with order between 5 and 9 inclusive. A computer check of all of these graphs (together with adding a light component) reveals the 72 obstructions indicated. 

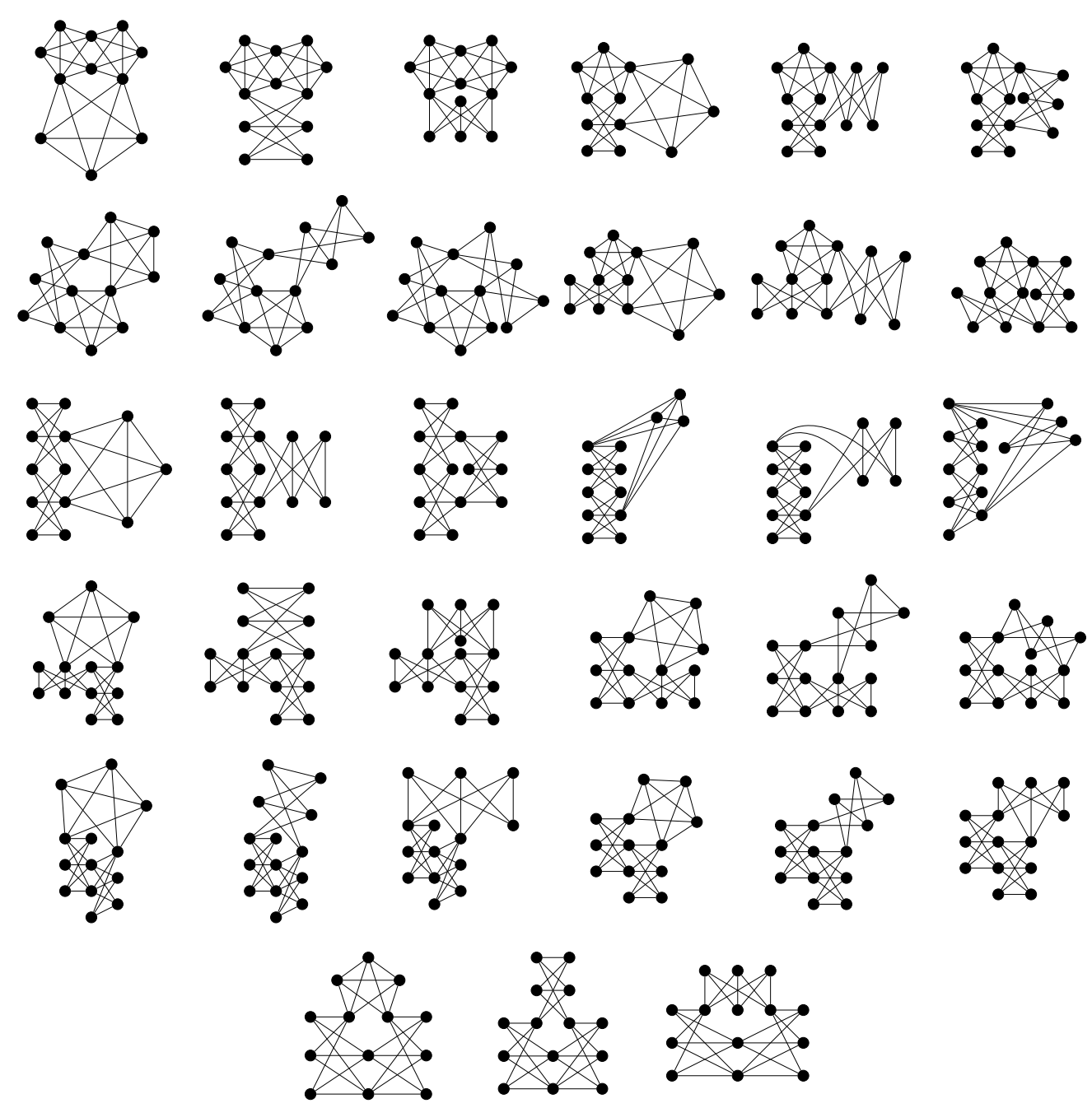

Figure 61: The 33 connectivity-2 apex obstructions that have a unique 2-cut $\{a, b\}$, a planar heavy component $C$, and a 2-cut separating $a$ from $b$ in $G[C \cup\{a, b\}]$.

\section{$9 \quad$ A double apex graph interpretation}

In this section we discuss another interpretation of our characterization of connectivity-2 apex obstructions because it may be of independent interest.

A graph $H$ with vertices $a$ and $b$ is double apex (with respect to $a$ and $b$ ) if $H-a$ and $H-b$ are non-planar, but $H-a-b$ is planar. Vertices $a$ and $b$ are the roots of $H$. For example, consider the rooted graphs obtained from $K_{5}$ and $K_{3,3}$ by replacing an edge with two subdivided edges and making the degree-two vertices the roots; denote these two graphs $K_{5}^{*}$ and $K_{3,3}^{*}$, respectively. These graphs (shown in Figure 63 with the roots colored red) are double apex. 

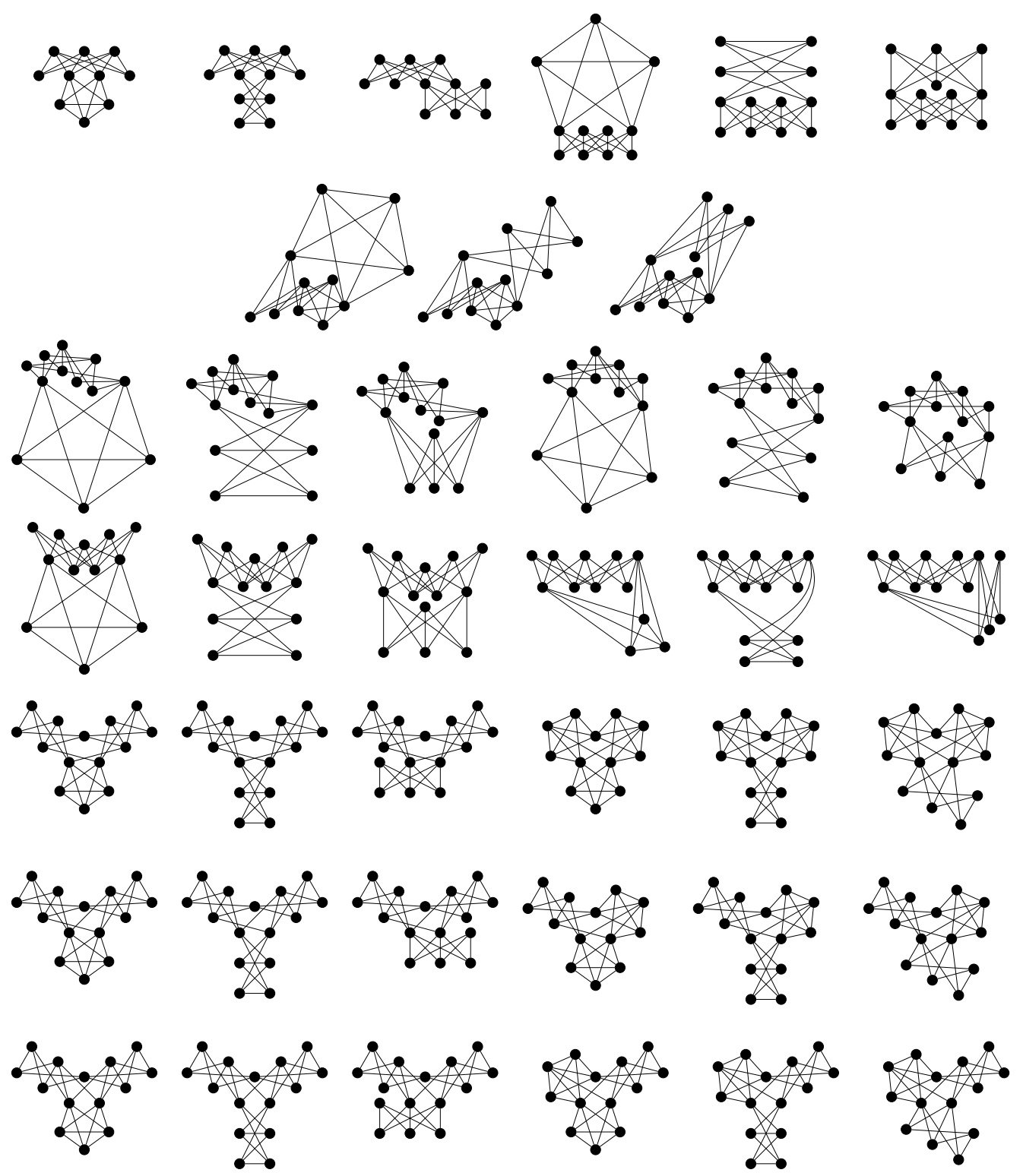

Figure 62: The 39 connectivity-2 apex obstructions that have a unique 2-cut $\{a, b\}$, a planar heavy component $C$, and no 2-cut separating $a$ from $b$ in $G[C \cup\{a, b\}]$. 

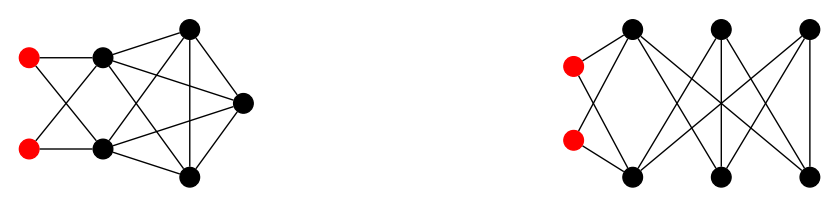

Figure 63: The two rooted double apex graphs $K_{5}^{*}$ and $K_{3,3}^{*}$.

Characterizing minor-minimal double apex graphs is a special type of 'intertwine' problem in which the goal is to determine minimal graphs that contain two Kuratowski subgraphs, each containing one root but avoiding the other. Note that $K_{5}^{*}$ and $K_{3,3}^{*}$ are minor-minimal double apex graphs. Recall that the Petersen family of graphs consists of the seven graphs: $K_{6}, K_{1,3,3}, Y^{-}, K_{4,4}-e, M, P_{7}, P$. Significant minor-minimal double apex graphs can be obtained from one of the seven Petersen family graphs by removing an edge and making its endpoints the roots. Note that not all such edge deletions produce a minor-minimal double apex graph $\left(K_{1,3,3}, Y^{-}, K_{4,4}-e\right.$, and $M$ have problematic edges).

One could restate Corollary 22 in the language of double apex graphs this way:

Theorem 27. Suppose that $H$ is a minor-minimal double apex graph with roots a and b. If $H+\{a b\} \neq Y^{-}, M, P_{7}, P$ and $H \neq K_{5}^{*}, K_{3,3}^{*}$, then a (resp. b) is a branch vertex of every Kuratowski subgraph in $H-b$ (resp. $H-a)$.

Using Theorem 27 one can prove (see the proof of Theorem 25) that in any minorminimal double apex graph $H$ satisfying $H+\{a b\} \approx Y^{-}, M, P_{7}, P$ and $H \approx K_{5}^{*}, K_{3,3}^{*}$, two Kuratowski subgraphs exist, one avoiding $a$ and the other avoiding $b$, whose branch vertices cover the entire vertex set. In this way all minor-minimal double apex graphs can be enumerated. Indeed it follows from our characterization of connectivity- 2 apex obstructions, that there are 57 non-isomorphic (as rooted graphs!) minor-minimal double apex graphs. Three are disconnected graphs; these are rooted versions of $2 K_{5}, 2 K_{3,3}$ and $K_{5}+K_{3,3}$ in which a root appears in each component. Twelve can be obtained from Petersen family graphs by removing an edge. For example, there are four non-isomorphic rooted minor-minimal double apex graphs that can be obtained from $M$ by removing an edge. The remaining 42 non-isomorphic rooted minor-minimal double apex graphs (including $K_{5}^{*}$ and $K_{3,3}^{*}$ ) can be found by inspecting the augmented heavy components of the connectivity-2 apex obstructions: see Appendix B and consider the 42 'cards' shown there in which all three 2-sums (with $K_{5}, K_{3,3}$, or $K_{3,3}+e$ ) produce an apex obstruction.

Assuming Theorem 27, it is easy to reason that if $H$ is a minor-minimal double apex graph with roots $a$ and $b$ and $H+\{a b\}$ is not isomorphic to a Petersen family graph, then a 2-sum at the roots $a$ and $b$ of $H$ with $K_{5}$ or $K_{3,3}$ will produce a connectivity-2 apex obstruction. Indeed Lemma 9 states that, under the right circumstances, a connectivity-2 apex obstruction must be generated in this way. So it is tempting to believe that knowing the 57 non-isomorphic rooted minor-minimal double apex graphs suffices to generate all connectivity-2 apex obstructions via such 2 -sums with $K_{5}$ and $K_{3,3}$. But these 2 -sums may generate the same graph more than one way. More significantly, because of non-planar heavy components or vertices that are not branch of any Kuratowski subgraph, there are 23 apex obstructions that can not be generated as a 2-sum in this way: nineteen of the 21 
graphs in Figure 5 and four graphs at the bottom of Figure 8. The two left-most graphs in the middle rows of Figure 5 can be generated from a 2 -sum applied to $K_{5}^{*}$ and $K_{3,3}^{*}$, respectively. Incidentally it is worth mentioning that, among the 23 apex obstructions that can not be generated as a 2-sum but excepting the seven graphs that contain vertices that are not branch of any Kuratowski subgraph, the remaining 16 graphs require only the branch vertices of two Kuratowski subgraphs to cover the entire vertex set.

\section{Acknowledgments}

We thank Csaba Biro and Tim Pervenecki for stimulating discussions and the referees for their thoughtful comments and suggestions. We are also very grateful to the editors for alerting us to references $[3,12,15]$.

\section{References}

[1] I. Adler, Open problems related to computing obstruction sets (Sep 13, 2008), https://pdfs.semanticscholar.org/dcb4/ 847bba47b1e1c2a0250013692d07f4bcc189.pdf.

[2] I. Adler, M. Grohe, and S. Kreutzer, Computing excluded minors, Proceedings of the Nineteenth Annual ACM-SIAM Symposium on Discrete Algorithms, ACM, New York, 2008, pp. 641-650. MR2487633

[3] H. Ayala, MMNA graphs on eight vertices or fewer (June 2, 2020), http://csuchico-dspace.calstate.edu/handle/10211.3/128451.

[4] R. Bodendiek and K. Wagner, On almost-planar graphs, Graphentheorie und ihre Anwendungen (Stadt Wehlen, 1988), Dresdner Reihe Forsch., vol. 9, Päd. Hochsch. Dresden, Dresden, 1988, pp. 9-12. MR979099

[5] G. Ding and S. Dziobiak, Excluded-minor characterization of apex-outerplanar graphs, Graphs Combin. 32 (2016), no. 2, 583-627, DOI 10.1007/s00373-015-1611-9. MR3461970

[6] G. Ding, J. Fallon, and E. Marshall, On almost-planar graphs, Electron. J. Combin. 25 (2018), no. 1, Paper 1.55, 14. MR3785034

[7] S. Dziobiak, Excluded-minor characterization of apex-outerplanar graphs, Ph.D. Thesis, Louisiana State University, 2011.

[8] D. Eppstein, $Y \Delta Y$-reducibility, apex graphs, and forbidden minors (Jul 23, 2010), https : //11011110.github.io/blog/2010/07/23/yy-reducibility-apex-graphs.html.

[9] _ More forbidden minors for apex graphs (Jul 26, 2010), https://11011110.github.io/blog/2010/07/26/more-forbidden-minors.html. 
[10] A. C. Giannopoulou and D. M. Thilikos, Optimizing the graph minors weak structure theorem, SIAM J. Discrete Math. 27, no. 3, 1209-1227, DOI 10.1137/110857027. MR3072757

[11] B. S. Gubser, A characterization of almost-planar graphs, Combin. Probab. Comput. 5 (1996), no. 3, 227-245, DOI 10.1017/S0963548300002005. MR1411084

[12] M. Lipton, E. Mackall, T. W. Mattman, M. Pierce, S. Robinson, J. Thomas, and I. Weinschelbaum, Six variations on a theme: almost planar graphs, Involve 11 (2018), no. 3, 413-448, DOI 10.2140/involve.2018.11.413. MR3733966

[13] A. E. Kézdy, All minor-minimal apex obstructions that have connectivity two (April 2013), http://www .ams.org/amsmtgs/2210_abstracts/1089-05-209.pdf. invited talk at the AMS Special Session on Extremal Graph Theory, University of Colorado, Boulder, CO (meeting \#1089).

[14] D. Marx and I. Schlotter, Obtaining a planar graph by vertex deletion, Algorithmica 62 (2012), no. 3-4, 807-822, DOI 10.1007/s00453-010-9484-z. MR2871126

[15] M. Pierce, Searching for and Classifying the Finite Set of Minor-Minimal Non-Apex Graphs (June 2, 2020), http://tmattman.yourweb.csuchico.edu/mpthesis.pdf.

[16] N. Robertson and P. Seymour, Graph minors. XX. Wagner's conjecture, J. Combin. Theory Ser. B 92 (2004), no. 2, 325-357, DOI 10.1016/j.jctb.2004.08.001. MR2099147

[17] N. Robertson, P. Seymour, and R. Thomas, Hadwiger's conjecture for $K_{6}$-free graphs, Combinatorica 13 (1993), no. 3, 279-361, DOI 10.1007/BF01202354. MR1238823

[18]__ Kuratowski chains, J. Combin. Theory Ser. B 64 (1995), no. 2, 127-154, DOI 10.1006/jctb.1995.1030. MR1339847

[19] _ Petersen family minors, J. Combin. Theory Ser. B 64 (1995), no. 2, 155-184, DOI 10.1006/jctb.1995.1031. MR1339848 


\section{A graph6 codes for connectivity-2 apex obstructions}

Computer-readable files of the graphs for Figures 5, 6, 8, 11, 61 and 62 can be found in the supplementary data file published with this article.

\section{B Augmented heavy components}
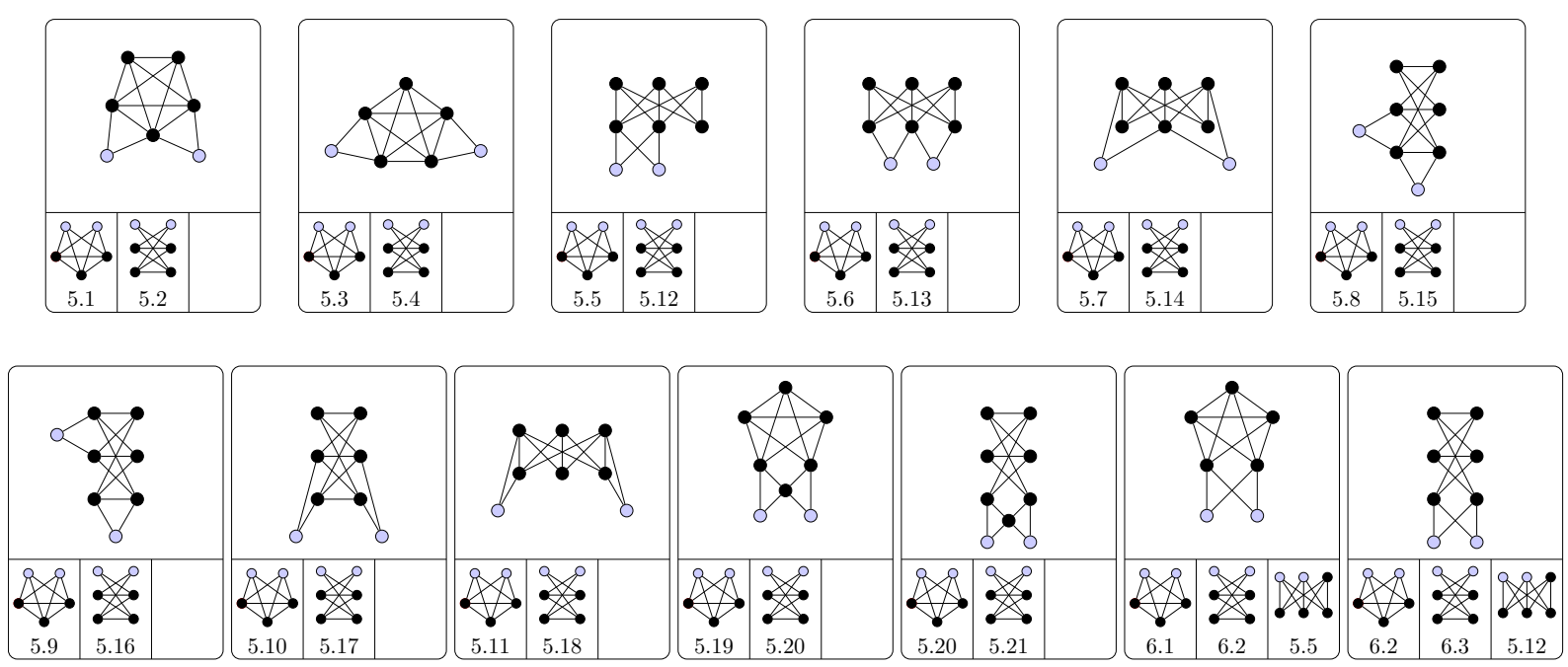

Figure 64: Augmented heavy components appearing in Figure 5 or Figure 6
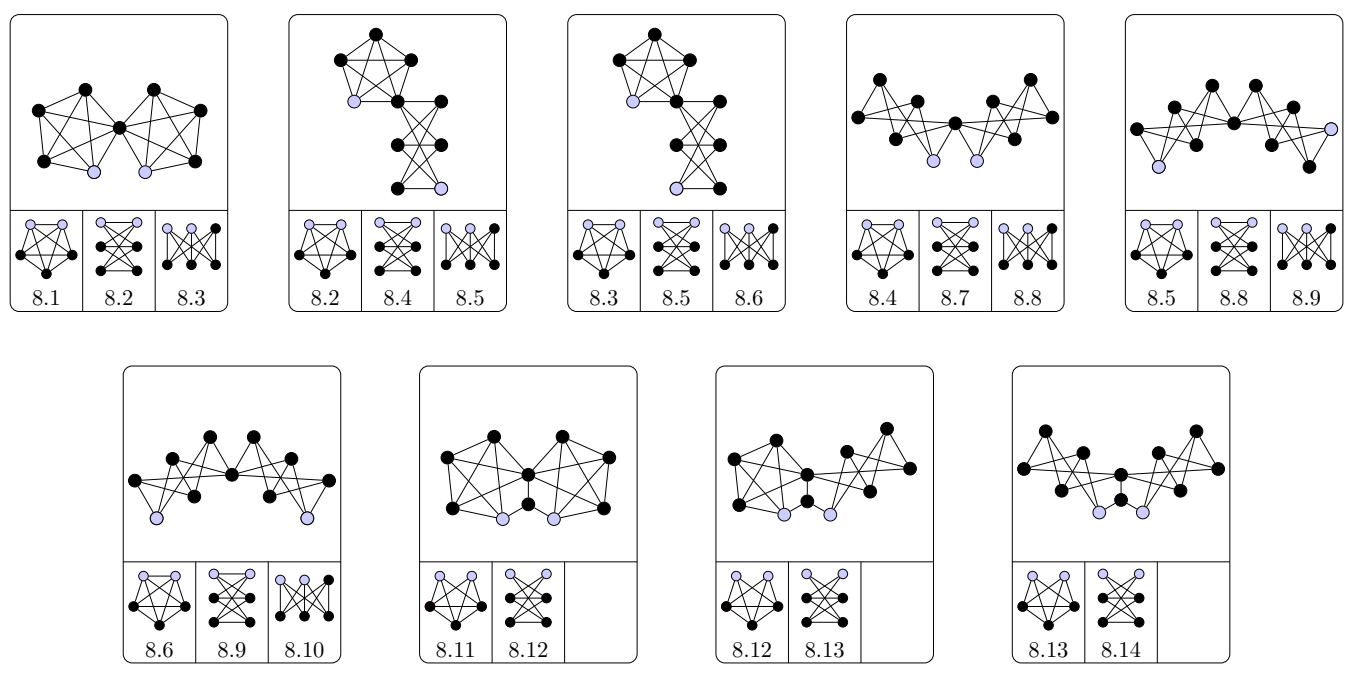

Figure 65: Augmented heavy components appearing in Figure 8 

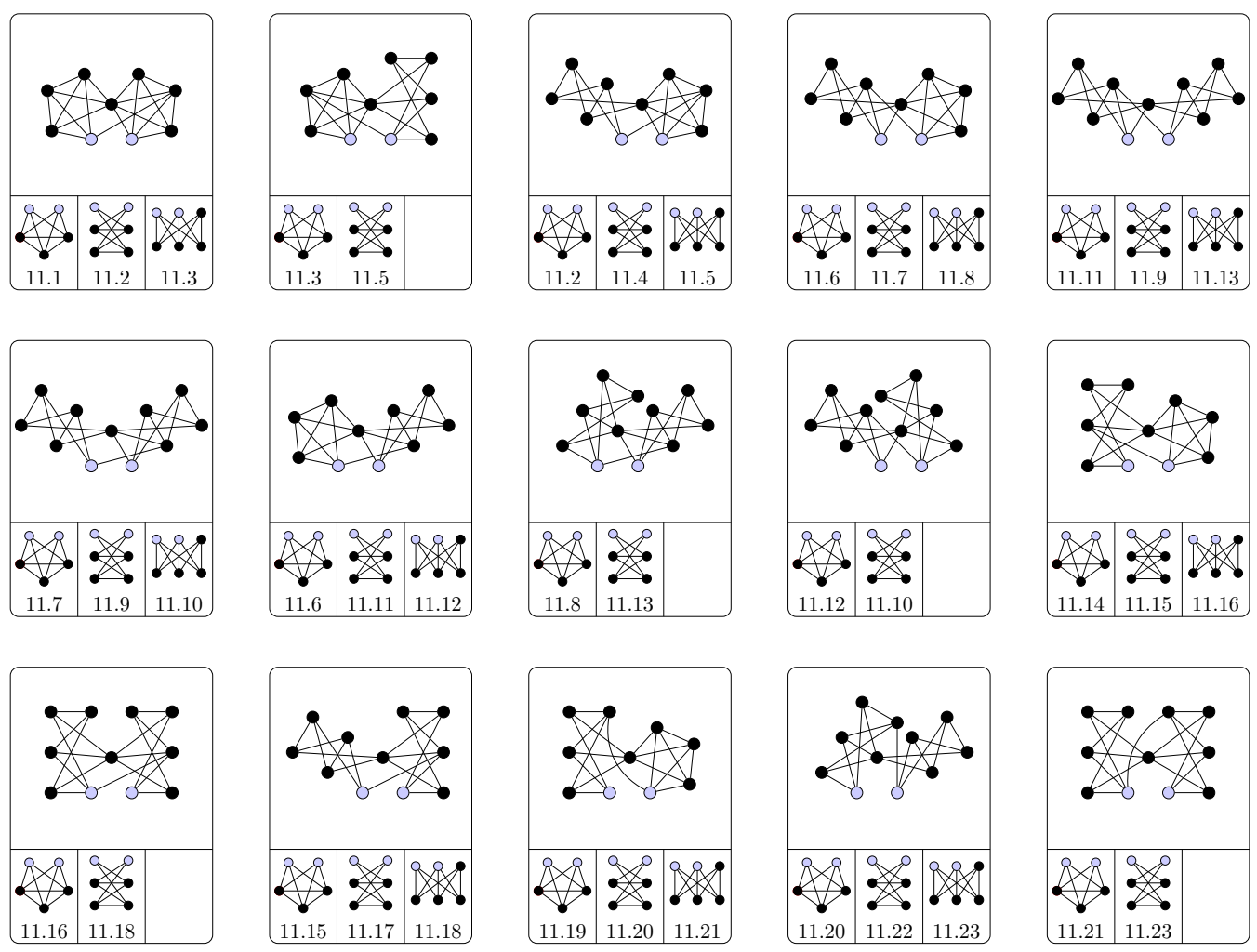

Figure 66: Augmented heavy components appearing in Figure 11
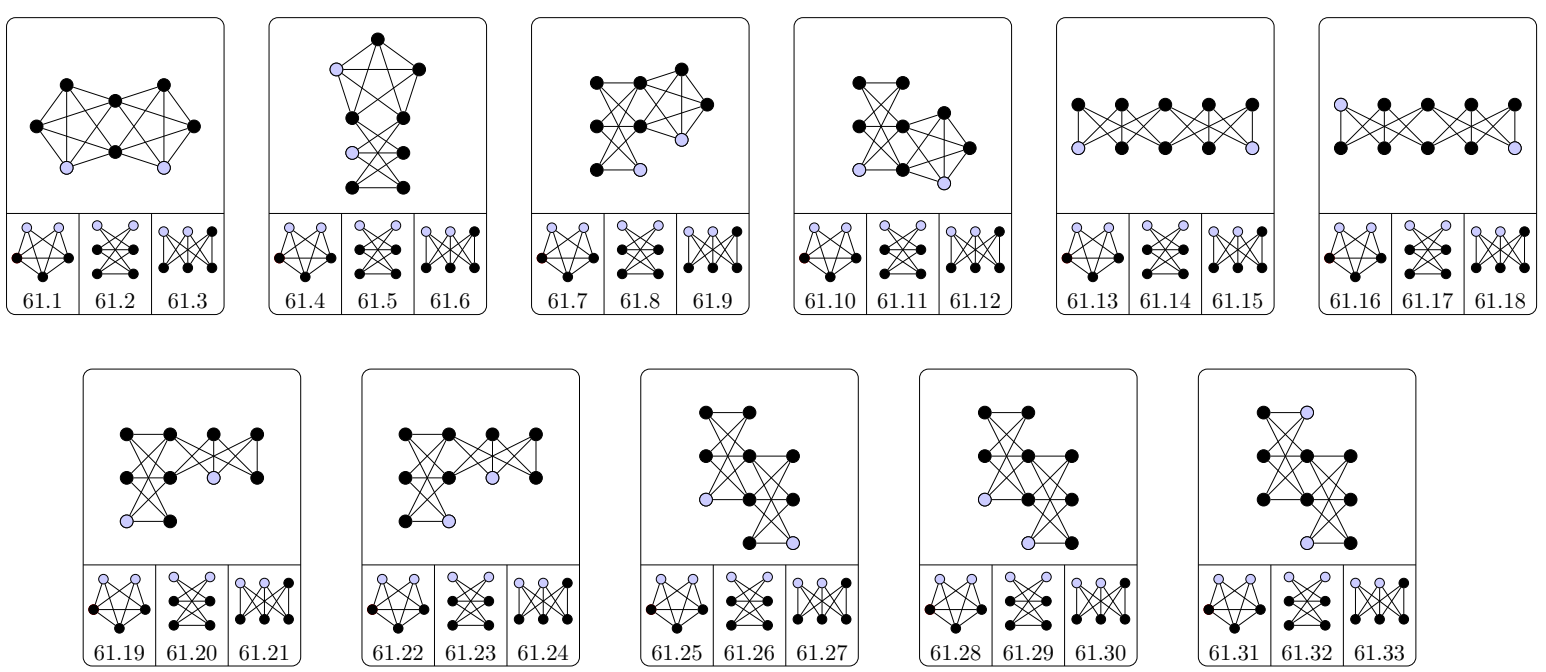

Figure 67: Augmented heavy components appearing in Figure 61 

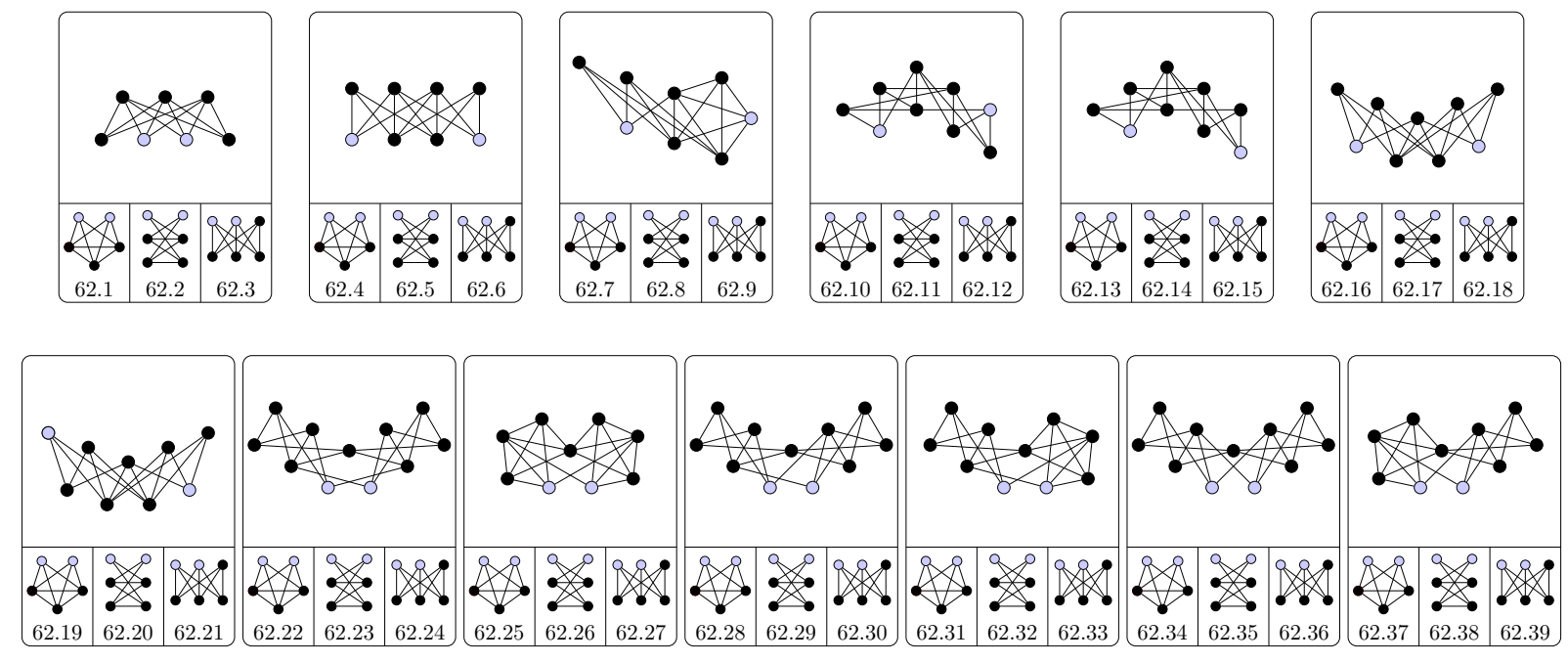

Figure 68: Augmented heavy components appearing in Figure 62 